
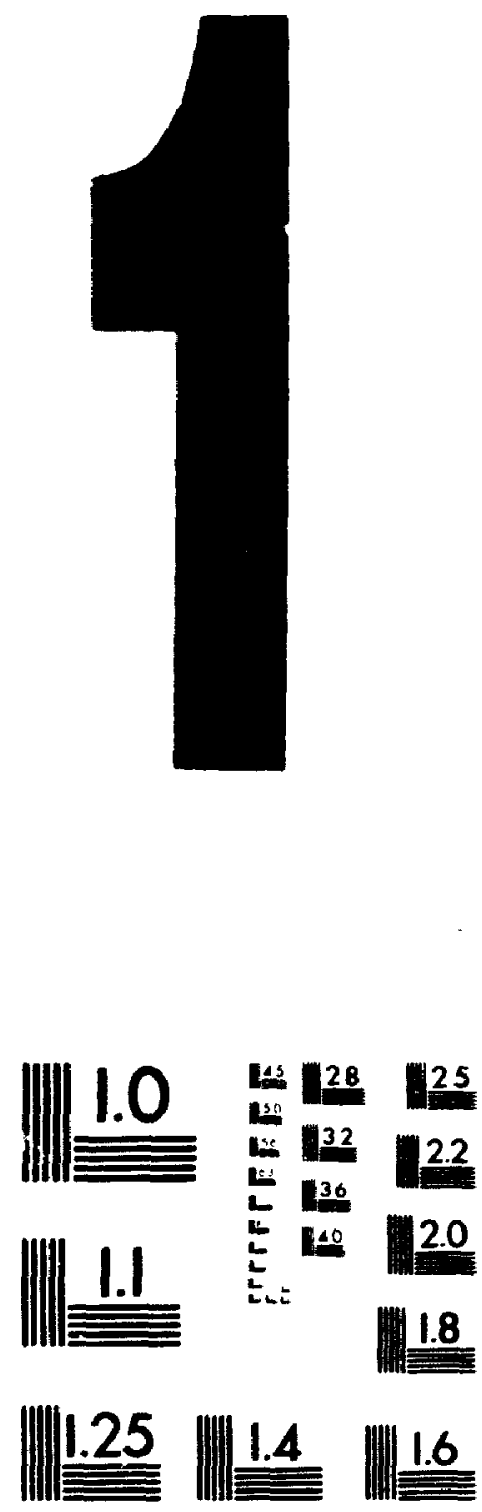

MICROCOPY RESOLUTION TEST CHART

NATIONAL BUREAU OF STANDARDS

STANDARD REFERENCE MATERIAL 1010a

(ANSI ard ISO TEST CMART NO 2) 
National Library

of Canada

Acquisitions and

Bibliographic Services Branch

395 Wellinglon Stree

Onawa. Ontano

KIA ONi4

\section{Bibliothèque nationale}

du Canada

Direction des acquisitions et

des services bibliographıques

395, ne Wellington

Otana (Ontano)

\section{NOTICE}

AVIS

The quality of this microform is heavily dependent upon the quality of the original thesis submitted for microfilming. Every effort has been made to ensure the highest quality of reproduction possible.

If pages are missing, contact the university which granted the degree.

Some pages may have indistinct print especially if the original pages were typed with a poor typewriter ribbon or if the university sent us an inferior photocopy.

Repruduction in full or in part of this microform is governed by the Canadian Copyright Act, R.S.C. 1970, c. C-30, and subsequent amendments.
La qualité de cette microforme dépend grandement de la qualité de la thèse soumise au microfilmage. Nous avons tout fait pour assurer une qualité supérieure de reproduction.

S'il manque des pages, veuillez communiquer avec l'université qui a conféré le grade.

La qualité d'impression de certaines pages peut laisser à désirer, surtout si les pages originales ont été dactylographiées à l'aide d'un ruban usé ou si l'université nous a fait parvenir une photocopie de qualité inférieure.

La reproduction, même partielle, de cette microforme est soumise à la Loi canadienne sur le droit d'auteur, SRC 1970, c. C-30, et ses amendements subséquenis. 


\title{
Performance Analysis of Client-Server Systems with Multi-threaded Clients
}

by

\author{
Jingping Lin
}

\author{
A thesis submitted to \\ the Faculty of Graduate Studies and Research \\ in partial fulfillment of \\ the requirements for the degree of \\ Master of Engineering
}

Ottawa-Carleton Institute for Electrical Engincering

Faculty of Engineering

Department of Systems and Computer Engineering

Carleton University,

Ottawa, Ontario, Canada

January 12, 1994

(c) copyright

1994. Jingping Lin 
National Library

of Canada

Acquisitions and

Bibliographic Services Branch

395 Wellingion Street

Onawa, Ontario

KIAON4
Bibliotheque nationale

du Canada

Direction des acquisitions et des services bibliographiques

395, ne Wellington

Ottawa (Ontarno)
The author has granted an irrevocable non-exclusive licence allowing the National Library of Canada to reproduce, luan, distribute or sell copies of his/her thesis by any means and in any form or format, making this thesis available to interested persons.
L'auteur a accordé une licence irrévocable et non exclusive permettant à la Bibliothèque nationale du Canada de reproduire, prêter, distribuer ou vendre des copies de sa thèse de quelque manière et sous quelque forme que ce soit pour mettre des exemplaires de cette thèse à la disposition des personnes intéressées.

L'auteur conserve la propriété du droit d'auteur qui protège sa thèse. Ni la thèse ni des extraits substantiels de celle-ci ne doivent être imprimés ou autrement reproduits sans son

autorisation.
The author retains ownership of the copyright in his/her thesis. Neither the thesis nor substantial extracts from it may be printed or otherwise reproduced without his/her permission. 
Dissertation Abstracts Internariagal is arranged by brood, general subject categories. Please select the one subject whict most

Dissertation Abstracty Internatropial is arranged by broad, general subject categories. Please select the one subject
nearty describes the content of your dissertation Enter the corresponding four-digit code in the spaces provided.

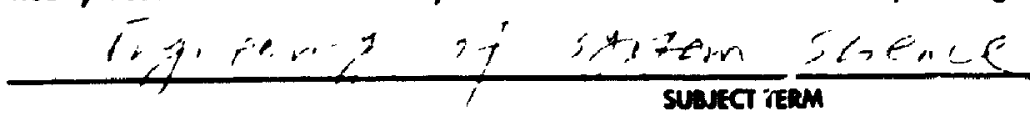

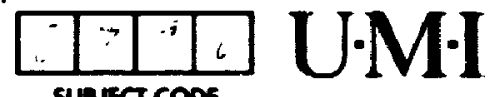

Subject Cologories

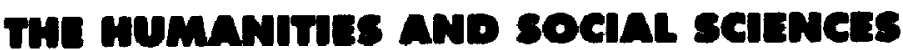

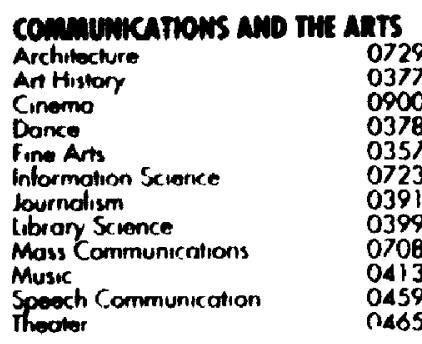

\section{soucamow}

Administrotion

Adult and Contirnung

Agr uculurd

An

Bu'ingual and Militiculthrol

Business

Community Collogo

Early Childhood

Elementriy

Fimunce

Health

Higher

History of

Home Economics

Industrial

Loriguage and lineruture

Maininmotacs

Music

Philosophy of

Physical

$\begin{array}{ll}\text { Psychology } & 0525 \\ \text { Reading } & 053 \\ \text { Religious } & 0527 \\ \text { Sciences } & 071 \\ \text { Sexondory } & 053 \\ \text { Sociol Sciences } & 053 \\ \text { Sociology of } & 034 \\ \text { Speciol } & 052 \\ \text { Teocher T.uining } & 053 \\ \text { Texhnology } & 071 \\ \text { Tests and Meosurements } & 028 \\ \text { Vocational } & 074\end{array}$

\section{UNGUAGE, UTERATURE AMD}

UMGUistics

0515

0514

0516
0517

0517
0273

0282

0688

0275

0518

0524

0524
0277

0519

0680

0745

0520

0278

0521

0279
0280

0280
0522

0998

0523
Language

General

lingyistics

Modern

Lterature

General
Classical

Comparative

Medieval

Modern

Alricon

American

Assum

Conodion (English)

Canodier (french)

English

Germanic

Latin American

Middle Eastern

Romonce
Slavtc and East European
PMILOSOPH', RELEGON ANO

\section{THEologr}

Philosophy

Religion

Biblical Studies

Clergy

History of

Philosophy of

Theology

socin scaences

American Studies

Anthropology

Archoootogy

Cultural

Business Administration

Generd

Accounting

Banking

Monagement

Marketing
Conodian Studies

Economics

Generol

Cornmerce-Business

Finance

History

Lobor

tolklore

Geogrophy

Gerontology

Histion

Generol

SUAJECT COOE

0422

0318

0321

0320

0322
0469

0323

0324

0326
0327

0310

0272

0454

0338

0385

$050^{\circ}$

0503

0508

0509

0510

0511

0358

0351

0578

\section{THE SeIENCIS AND ENGINERRINC}

\section{moloexcul satancts}

Agriculture

Agronomy

Animal Culture and

Nutition

Animal Pothology

trood Science and

Iechnology.

forestry and Wildite

Plont Culture

Plont rothology

Plont Phys ology

Range Manogement

Buology

Wood Technology

General

Anctomy

Boksnit

Cetl

Trologyy

Entomotagy

Conetrrs

Microbiology

Molocutar

Nourosinerice

Oceonogyruphy

Physsotogy

Rodiofion

Zonlogy

Mophysirs

Menoral

Madica

\begin{tabular}{|c|c|}
\hline $\begin{array}{l}0473 \\
0285\end{array}$ & $\begin{array}{l}\text { Goodesy } \\
\text { Geology } \\
\text { Geophysics } \\
\text { Hydrology }\end{array}$ \\
\hline $\begin{array}{l}0475 \\
0476\end{array}$ & Poleobotony \\
\hline $\begin{array}{l}0359 \\
0478 \\
0479 \\
0480 \\
0817\end{array}$ & $\begin{array}{l}\text { Paleozoology } \\
\text { Palynology } \\
\text { Physicol Geogrophy } \\
\text { Physical Oceanography }\end{array}$ \\
\hline
\end{tabular}

IARm satences

Bingoxchenistry
0817

0777

0746

0306
0287
0308

0308

0379

0329

0353

0369

0793

0410

0.317

0416

0433

0821

0878

0472

0780

\section{MEMTH AMD ENVIROM METTA}

\section{SaEnas}

Environmental Sciences

0768

Heolth Sciences

Genera

Audiology

Chemotherapy

Dentistry

Education

Human Developmen!

Immunology

Medicine aru Surgery 050

$\begin{array}{ll}\text { Mental Health } & 0347 \\ \text { Nursing } & 0569\end{array}$

Nursing

Nutrition

Obstetrics and Gynecology

Theropy

Pothology

Phormocology

Pharmocy

Public Heulth

0425

$090^{\circ}$

Red ology
0370

0372
0373

0373

0411

0345

0426

0418

0985

0427

0368
Speech Pothology

Home Economics

Pursieal sasmas

Pure Sciences

Chemistry

Agriculturo

Analytical

Biochemistry

Inorganic

Nuclear

Phormaceutical

Physical

Polymer

Mothematics

Physics

General

Acoustics

Astronomy and

Astrophysics

Almospheric Science

Atomic

Electronics and Electricit

Elemeniary Porticles and

High Energy

Molecular

Nucleas

Optics

Radiation

Solid Siate

Statistics

Applied Sciences

Applied Mechanics

Compuner Science
0460

0383
0386
0381

0419

0382

0573

0575
0485

Engineering
Generd

Acrospace

Automofive

Biomedical

Chemical

Civil 
The undersigned hereby recommend to

the Faculty of Graduate Studies and Research acceptance of the thesis

\section{Performance Analysis of Client-Server Systems with Multi-threaded Clients \\ submitted by \\ Jingping Lin, B.Eng}

In partial fulfillment of the requirements

for the degree of Master of Engineering

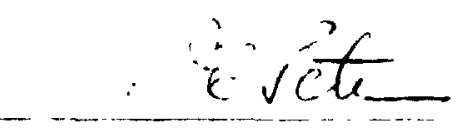

Thesis Co-Supervisor

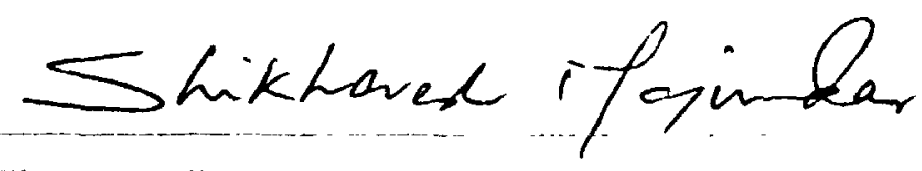

Thesis Co-Supervisor

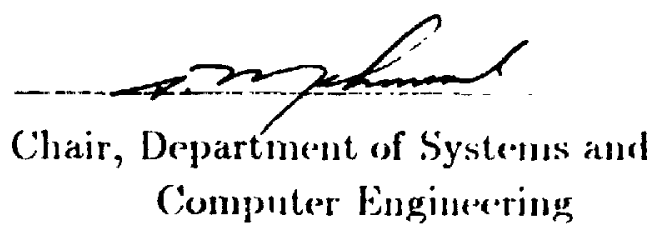

Carleton University

Jamuary 12,1994 


\section{Abstract}

This thesis introduces an analytical performance model named Rendezvous Network with Multi-Threaded Clients (RNMTC) fo: performance analysis of client/server system. RNMTC is an extension of the Stochastic Rendezvous Network (SRVN), a previously developed model for tasking systems witt: rendezvous ( $R V$ ) communication style. An approximate analytical solution method and a simulator were developed for RNMTC and implemented in the thesis. Techniques for the computation of performance bounds for the RNMTC model are also investigated.

The analytic solution of the RNMTC model is based on hierarchical decomposition. At the higher level, a Markov Chain (MC) model is built such that the MC states correspond to all possible combinations of client execution states; at the lower level, each MC state is represented by an SRVN model with simple clients.

The analytic solution for RNMTC is applied to a number of test cases, the most important being a large case study of a distributed database running on a Tandem system. The analytic results are compared with two simulation results: one from a "detailed simulation model" which captures the hardware architecture of a Tandem computer system and the software behavior of the relational database system, and one from an "abstract simulation model" used to ana! yze RNMTC models. The "abstract error" is defined as the difference between the detailed and abstract simulation results while the "analytic approximation error" is defined as the difference between the abstract simulation and analytic results.

Performance bounds that are based on a set of very loose assumptions about the stochastic behaviour of the system are investigated. Techniques for the computation of an upper abound and a lower bound on performance for a number of specific RNMTC models are presented. 


\section{Acknowledgements}

First, I would like to express my deep gratitude to my supervisors. Dr. D. Petriu and Dr. S. Majumdar, for their valuable guidance, advice and encouragement which they have given me throughout the research.

I would like to thank C. Hrischuk for his help with the Tandem Database system simulation.

The support and assistance from the secretaries in department of systems and computer engineering are also greatly appreciated.

I would also like to thank Carleton University and the Natural Sciences and Engineering Research Council of Canada for their funding support.

Finally, I wish to give my special apprexiation to my parents, my husband Wendell for his consistent support and encouragement and my son Bo for his patience and understanding. 


\section{Table of Contents}

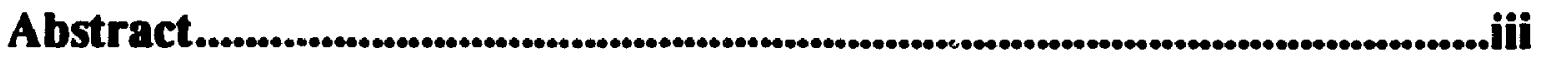

Acknowledgements......................................................................................iv

List of Figures................................................................................................................. viii

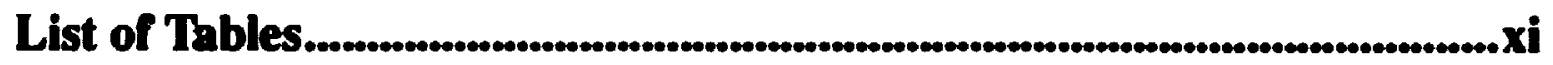

List of Symbols and Terminology ......................................................................xii

1. Introduction

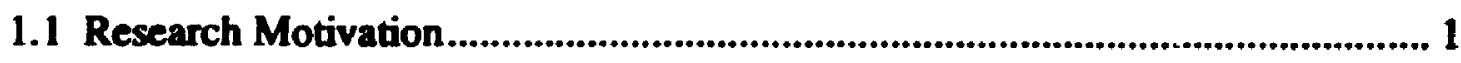

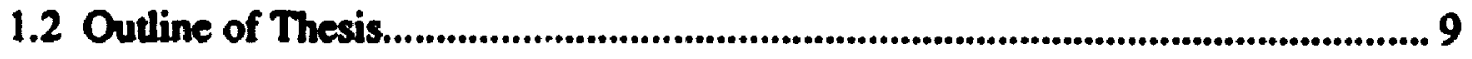

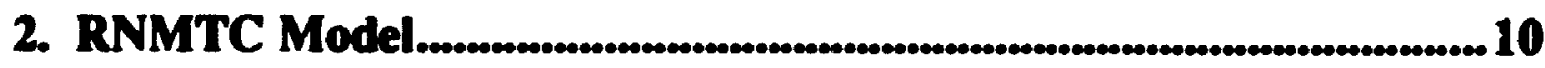

2.1 The SRVN Model .................................................................................................. 10

2.2 Definition of the RNMTC model....................................................................... 15

2.2.1 Client Precedence Graph ......................................................................... 15

2.2.2 RNMTC Model Parameters........................................................................... 19

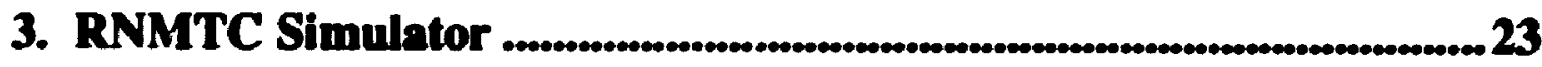

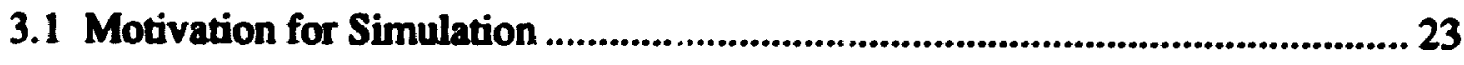

3.2 Brief Introduction to PARASOL .................................................................... 24

3.3 Architecture and Implementation of the RNMTC Simulator............................ 25

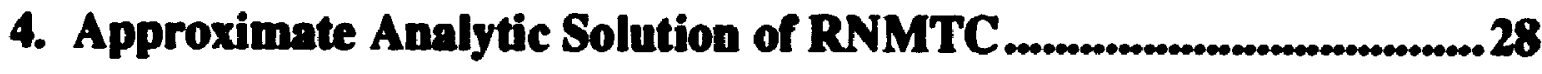

4.1 Solution Introduction ................................................................................................. 28 


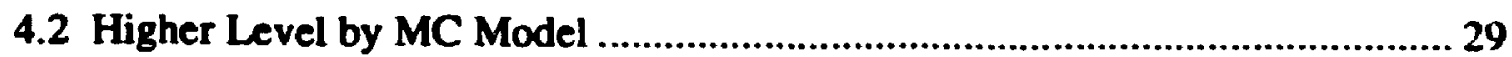

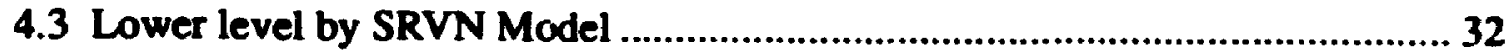

4.4 Decomposition Method .................................................................................... 34

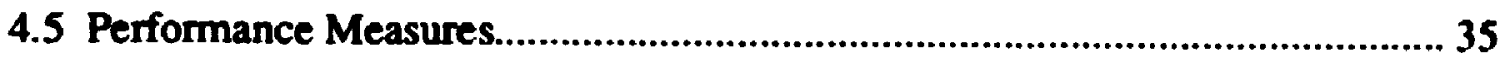

4.5.1 Performance Measures for Single Client RNMTC System.................... 35

4.5.2 Performance Measures for Multiple Clients RNMTC System................ 37

5. Bounds.

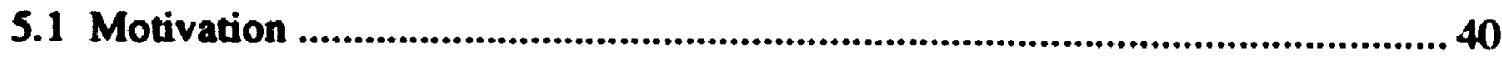

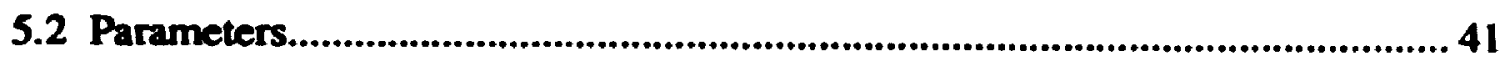

5.3 Bounds on the Performance of Deterministic System......................................4 44

5.3.1 Bounds on Systems with Fixed Computation Times and Service

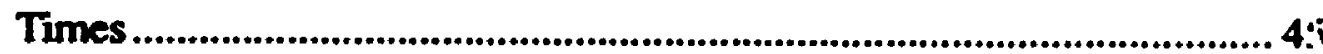

5.3.2 Bounds on Systems with General Computation and Service Time Distribution......................................................................................................... 48

5.4 Bounds on the Performance of Systems with Probabilistic Branching ............ 54

5.5 Bounds on System with Simple Loops.............................................................60

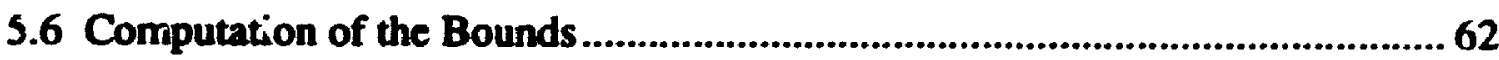

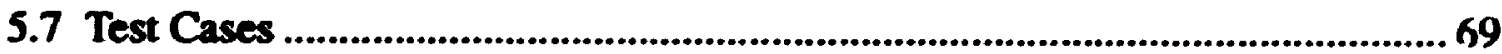

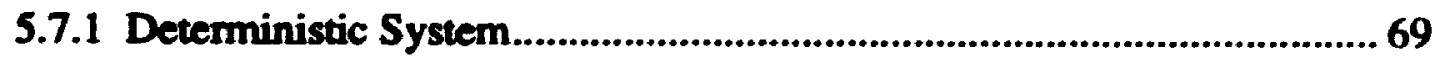

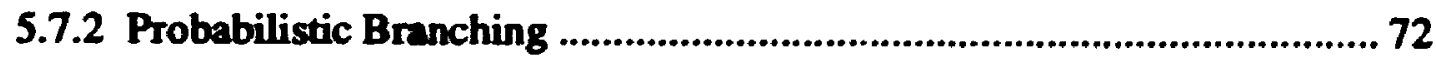

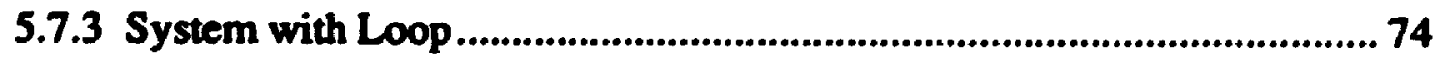

6. Test Cases for Analytic Solution Method................................................ 77

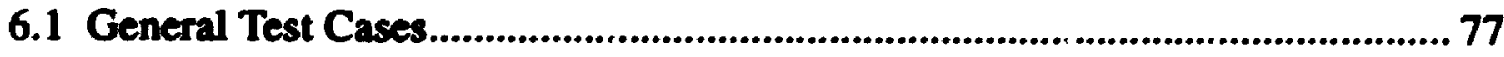

6.1.1 Model 1: Balanced System ....................................................................... 78

6.1.2 Model 2: Impact of the Number of Concurrent Tasks........................... 79 
6.1.3 Model 3: Impact of layer of Client Tasks ............................................ 80

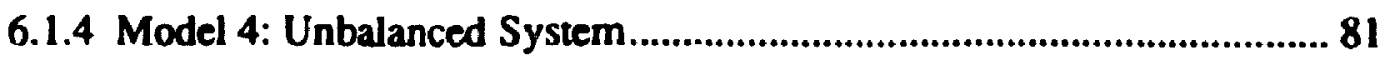

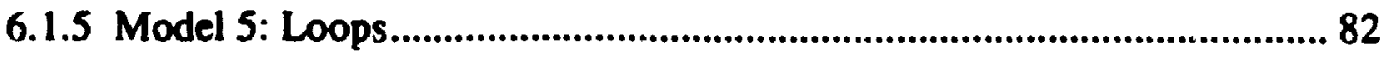

6.1.6 Model 6: Probabilistic Branching............................................................... 84

6.1.7 Model 7: Processor Contention ........................................................................ 85

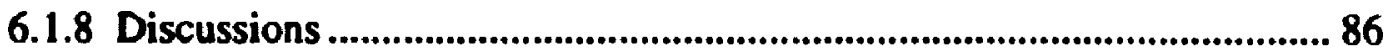

6.2 RNMTC Model of a Distributed Database System ............................................ 91

6.2.1 Tandem Distributed Database System .................................................. 91

6.2.2 RNMTC model for Tandem System....................................................... 94

6.2.3 RNMTC Model for Light Reader Operation ........................................... 95

6.2.4 RNMTC Model for Join Query Operation ........................................... 98

6.2.5 Hardware and Software Bottleneck.................................................... 102

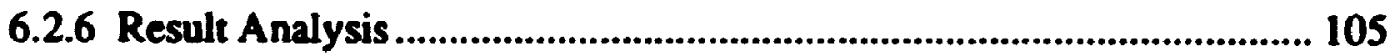

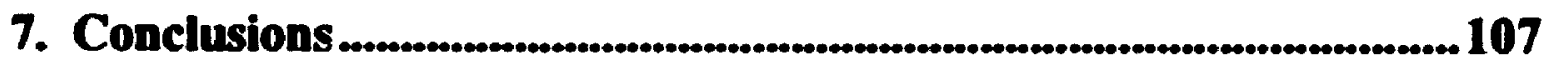

7.1 Research Summary and Contributions .............................................................. 107

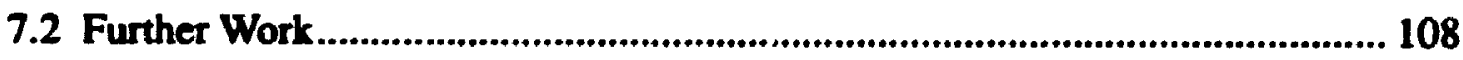

References

Appendix Usage of RNMTC Simulator and analytic Solver ................ 115 


\section{List of Figures}

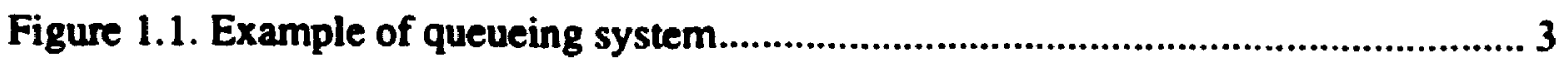

Figure 1.2. Flow model for a simple concurrent system ...............................................

Figure 1.3. Example of Petri-Net system...................................................................... 5

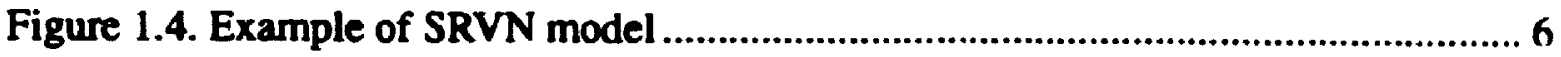

Figure 2.1. SRVN task behavior ................................................................................... 11

Figure 2.2. Example of an SRVN model ......................................................................... 12

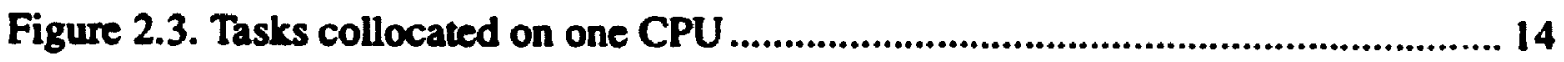

Figure 2.4. SRVN model for processor contention..................................................... 14

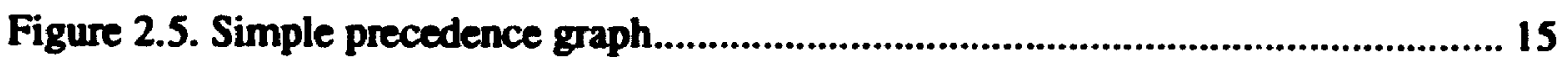

Figure 2.6. Edge terminating at the same node............................................................ 16

Figure 2.7. edge emanating from the same node .............................................................. 17

Figure 2.8. Loop relationship.................................................................................... 17

Figure 2.9. Simple precedence graph model.................................................................. 18

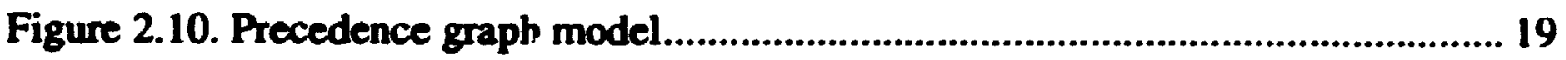

Figure 2.11. Simple RNMTC model............................................................................. 20

Figure 2.12. Multiple client RNMTC model .................................................................. 21

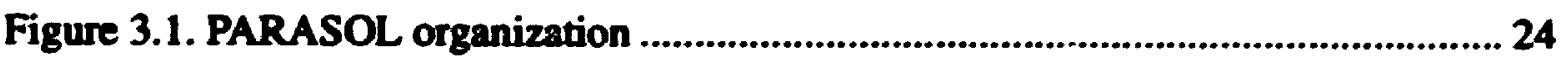

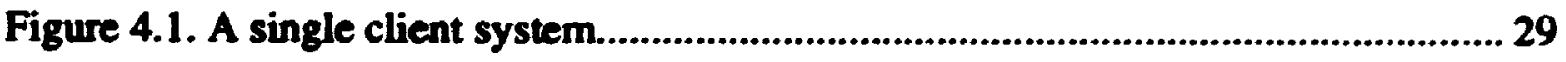

Figure 4.2. MC for simple system .............................................................................. 30

Figure 4.3. Precedence graph with multiple classes .....................................................30

Figure 4.4. MC for multiple client system ...................................................................... 31

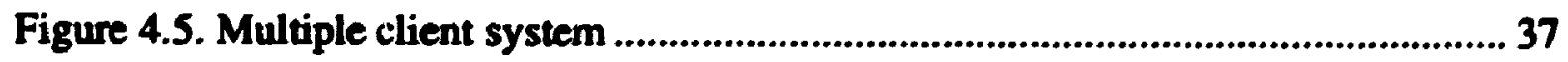




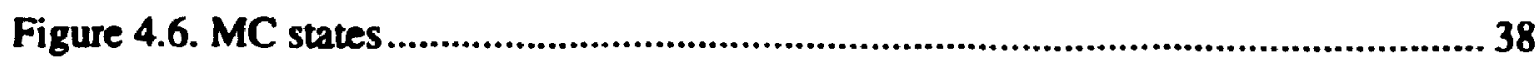

Figure 5.1. Concurrent task in unbounded system.......................................................48

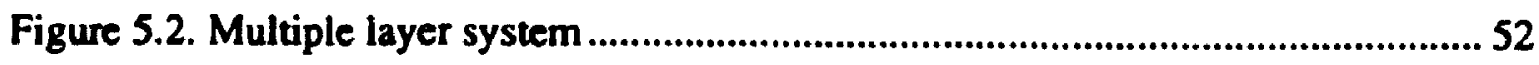

Figure 5.3. Simple probabilistic branching .............................................................54

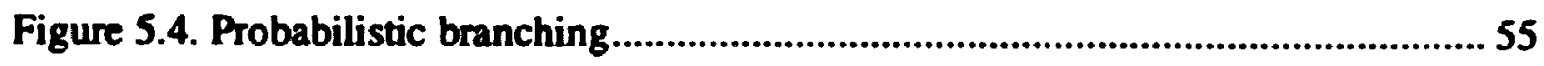

Figure 5.5. Multiple layer probabilistic branching system ............................................ 58

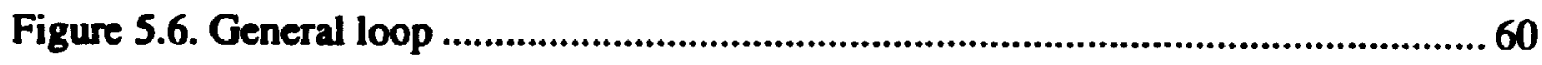

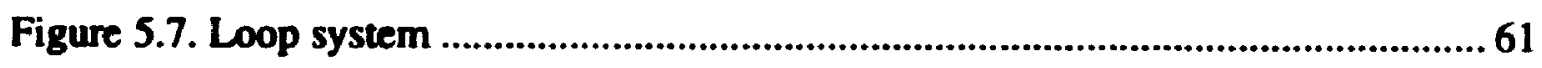

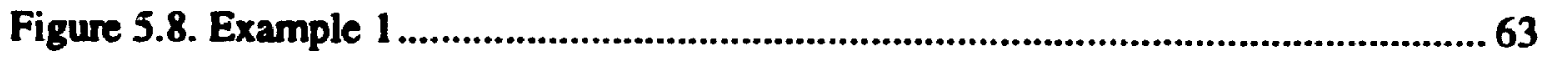

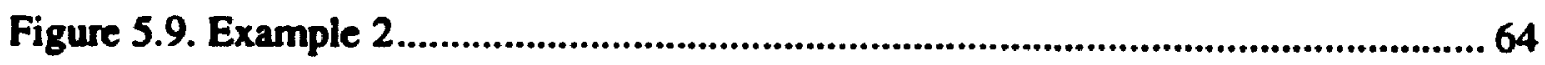

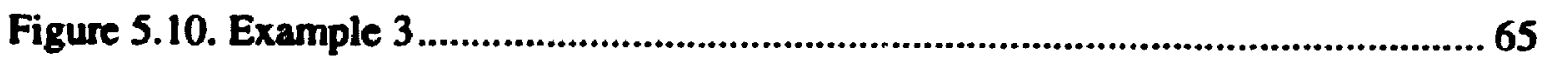

Figure 5.11. Test case of multiple layer systerr........................................................... 69

Figure 5.12. Test result for system with fixed cornputation time and service time ........ 70

Figure 5.13. Test case for exponential distribution....................................................... 71

Figure 5.14. Test case of probabilistic branching ...................................................... 72

Figure 5.15. Test result of probabilistir branching ....................................................... 73

Figure 5.16. Test case of loop system .......................................................................... 74

Figure 5.17. Test result for loop with 4 concurrent client tasks...................................... 75

Figure 5.18. Test result for loop with 8 concurrent client tasks..................................... 76

Figure 6.1. Balanced system .................................................................................... 78

Figure 6.2. Error rate for balanced system................................................................... 79

Figure 6.3. Impact of number of concurrent tasks .....................................................8 80

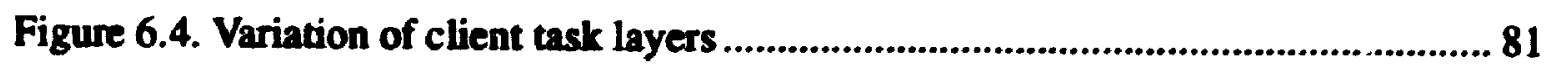

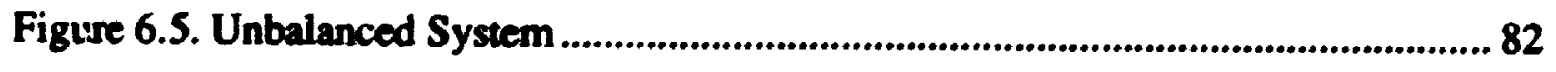




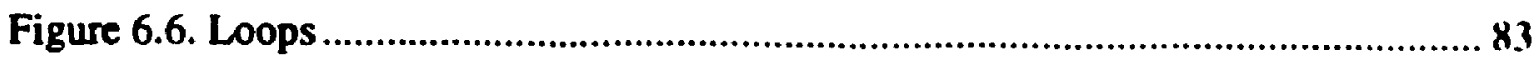

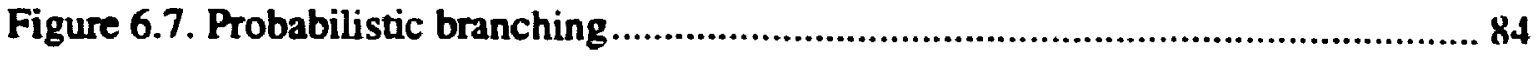

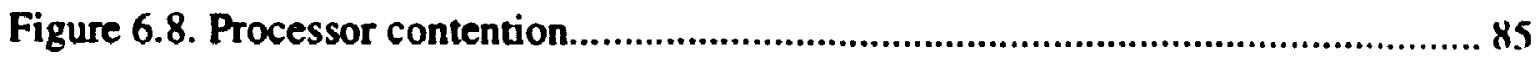

Figure 6.9. RNMTC model for processor contention ................................................... 8h

Figure 6.10. Tandem System Architecture ................................................................93

Figure 6.11. Light Reader Query Execution Model..................................................... 95

Figure 6.12. RNMTC model for light readers .......................................................... 96

Figure 6.13. Response time in function of the cache hit ratio .......................................97

Figure 6.14. Parallel join query execution model ........................................................... 99

Figure 6.15. RNMTC model for join query model.................................................... I(x)

Figure 6.16. RNMTC model for two classes .................................................................. 101

Figure 6.17. Response time for two classes of clients ................................................. 102

Figure 6.18. Light reader's response time in function of the number of clients.......... 103

Figure 6.19. Utilization of servers in case of hardware bottleneck ............................. 104

Figure 6.20. Utilization of servers in case of software bottleneck.............................. 105 


\section{List of Tables}

Table 1.1: Simulation and Approximate results for the Balanced System (Model 1)

Table 2.1: Simulation and Approximate results when the number of concurrent tasks $=3$ (Model 2$)$

Table 2.2: Simulation and Approximate results when the number of concurrent tasks $=5($ Model 2$)$

Table 2.3: Simulation and Approximate results when the number of concurrent tasks $=10$ (Model 2)

Table 3.1: Simulation and Apprcximate results for the impact of client task layers (Model 3)

Table 4.1: Simulation and Approximate results for the impact of processor contention (Model 7).

Table 5.1: Accuracy of Analytic Solution. 90 


\section{List of Symbols and Terminology}

RNMTC $=$ Rendezvous Networks with Multi-Threaded Client

SRVN = Stochastic Rendez Vous Network

$\mathbf{R V}=$ RendezVous

QN = Queueing Network

PN $=$ Petri Net

MC = Markov Chain

"in RV" phase = execution phase of an accepting task, when the requester is blocked is blocked waiting for a reply from the acceptor (also named first phase in SRVN and RNMTC)

"post RV" = execution phase of an accepting task, executed in parallel with the requester (also named second phase in SRVN)

$C_{a}=$ total number of classes in the client system

$C_{(}(i)=$ total number of clients in class $i$

$N(i)=$ total number of client tasks in one client of class i

$K=$ total number of servers in the system

$U(j)=$ total number of entries in server task $j$

$c(i, t)=$ average computation time for client task $t$ in class $i$ per job cycle

$s(i, e, p)=$ average service time in entry $e$ during phase $p(p=1$ or $p=2)$ of server task $i$ per visit

$S(i, e)=$ average service time in entry $e$ of server task $i$ in two phases per visit

$$
s(i, e)=s(i, e, l)+s(i, e, 2)
$$


$v(i, t, j, u(j))=$ average number of visits made by client task $t$ in class $i$ to entry $u$ of server task $j$ per job cycle

$v(i, t, j)=$ average number of visits made by client task $t$ in class $i$ to server task $j$ per job cycle

$$
v(t, t, j)=\sum_{u=1}^{U(j)} v(i, !, j, u(j))
$$

$y(i, e, p, j, d)=$ mean number of RV requests made by (server) task $i$ in entry $e$ during phase $p$ to entry $d$ in task (server) $j$. 


\section{Chapter 1}

\section{Introduction}

\subsection{Research Motivation}

Computer system performance in terms of speed and efficiency is one of the major factors which irfluence the design, development, configuration and tuning of a system. It is desirable to evaluate the performance of a computer system including both software and hardware components, because we want to make sure that the system is suitable for its intended applications, and that it satisfies the performance requirements. Performance evaluation is also useful to assess if a computer system operates near its optimal capacity under given resource constraints. With the enhancement in size and complexity of current computer systems, performance evaluation becomes more and more important and is strongly required throughout the life time of a system.

There are a number of system performance measures, such as, cycle time, response time, throughput, utilization, speedup, reliability, etc. The specific performance measures of interest is dependent on the types of the system. For example, response time is more crucial than other performance measures in a real time system, whereas throughput is a better description of performance for a batch processing system.

Different performance evaluation techniques may be appropriate in different stages of the lifetime of the system. For example, measurement is an appropriate approach once a system is built and instrumented, whereas modelling may be the only performance evaluation technique that may be used before the construction of the system. This thesis is concerned with the second approach to performance evaluation. 
A model is an abstract representation of the system under study. By analyzing and evaluating the model, the behavior of the system can be inferred. An appropriate model should be simpler than the real world and relatively easy to analyze. Therefore, the construction of a model requires a process of considerable abstraction. Some characteristics of the system must be kept in the model because they are the major factors which affect the performance of the system. On the ther hand, some other characteristics may be ignored to make the model simpler, if they have less impact on system performance. Unfortunately, there is no explicit definitions about weather a feature should be included in the model or not. The building of an appropriate model is mostly dependent on the ability and experience of the modeler.

In terms of modeling, the simulation modeling plays an important practical role in performance evaluation. More details of a system can be aptly captured in a simulation model than in analytical models and thus the simulation results are closer to the real world. However, the disadvantage of simulation is its high cost of developing the models and of running the experiments.

Using inexpensive analytical models may be a viable altemative to the analyst or designer for estimating system performance. Analytical modelling has the following advantages:

- It has much lower cost than simulation modeling.

- It provides insight on the performance characteristics of the system under study.

- Compared to the measurement methods which can be applied only to existing systems, analytical modeling methods not only can evaluate an existing system, but also can predict the performance of a future system.

Analytical modeling has also some disadvantages. It may have lower accuracy than simulation due to modeling restrictions imposed on analyival models to insure their feasibility and simplicity. Also, the size of the model has a strong impact on the complexity of analytical solutions, which makes some methods intractable for systems of a practical size.

A brief review of some known analytical models is given in the following.

Queueing Nerwork (QN) is a well developed practical tools for computer system performance analysis [Lav89] [LZGS84]. A QN includes service centers which normally represent hardware resources, and clients which represent software jobs. The service centers are shared by 
the clients. Each slient obtains service for a given interval of time from a service center. After the service, the client leaves the service center and then may proceed to another service center. Each service center is associated with a queue in which the clients wait for service. There may be multiple service levels in each server queue and different service disciplines, such as FIFO (first come, first served), PS (processor sharing), may be used. Clients can be divided into different classes. Clients with statistical identical characteristics, such as routing probabilities, service demand, are put into the same class. The only dependence among clients is that they compete for server resources. The clients do not synchronize or communicate with one another. If the number of clients in the QN system is fixed, the QN is called closed. On the other hand, an open QN allows clients that complete their servise to depart from the system and allows new client(s) to enter the system. Figure 1.1 is an example of a queueing system in which the CPU and the VOO devices are modelled by different kinds of service centers.

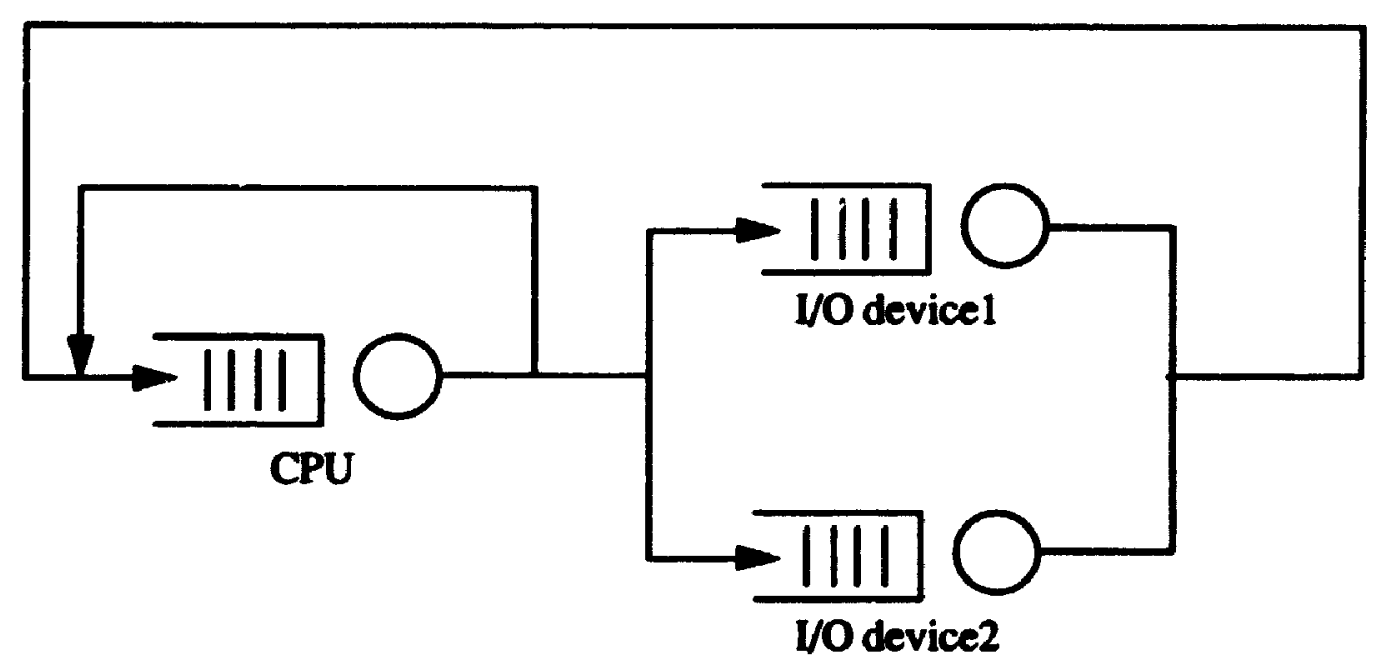

Figure 1.1. Example of queveing system

Numerous solution methods to solve queueing models have been developed. A survey of analytical computer performance modeling technique with an emphasis on developments of prac- 
tical importance can be found in [Lav89].

QN is a powerful analytical model to evaluate system performance, but has also limitations [VZL87]. It fails to capture the impact of system concurrence and the communication and synchronization among software jobs, since a QN server center plays a sole role: supplying service. In a $\mathrm{QN}$, each client is served by one service center at a time. The multi-level service requests produced by a server which calls another server while serving a client cannot be modeled directly by QN.

A Petri-Net (PN) is another kind of analytical model [AMBC84] [Mol82]. A PN contains a set of places, a set of transitions and a number of tokens. The tokens reside in places and may move from one place to another caused due to the firing of transitions. A. PN is very efficient in representing flow models of concurrent software. Figure 1.2 shows a flow model for a simple system with concurrent tasks.

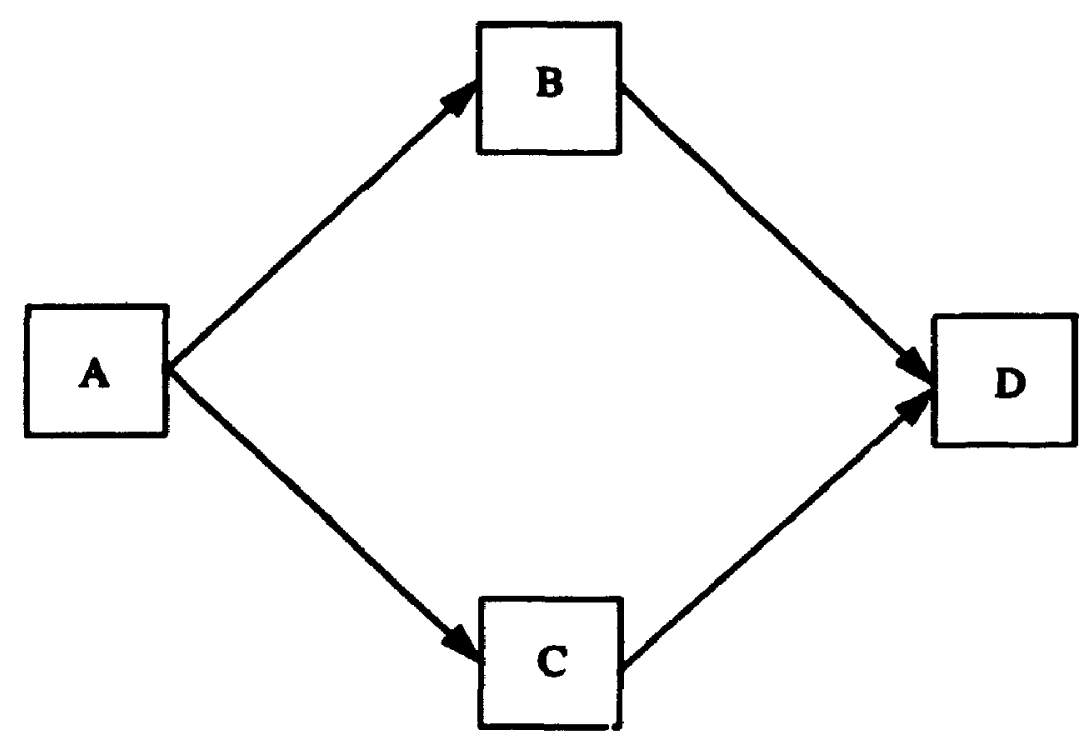

Figure 1.2. Flow model for a simple concurrent system

Figure 1.3 is a simple example of a PN system that is equivalent to the structure of Figure 1.2. 


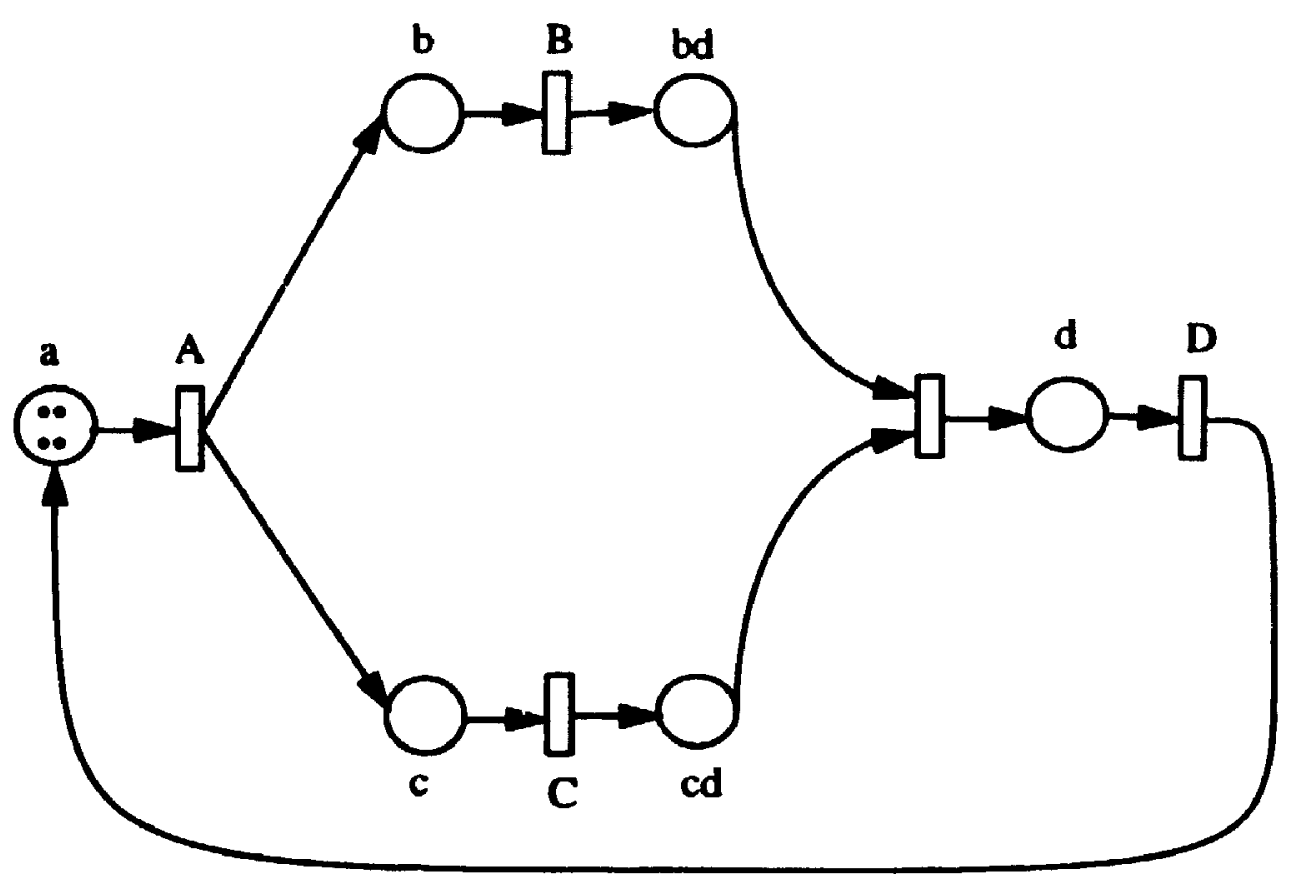

Figure 1.3. Example of Petri-Net system

The disadvantage of a PN is that it is weak in modeling contention for resources and may give rise to the well known problem of state space explosion.

Stochastic Rendezvous Networks (SRVN) in [MWNP88] [MWNP89] [PW91] [WNMP88] [WNPM89] and [Woo89] is a relatively new model which combines the advantages of both QNs and PNs. A SRVN is very appropriate in modelling clien/server systems with rendezvous (RV) style of communication among tasks. Since this thesis extends SRVN, the mudel is presented in more details than QN and PN models.

Intertask rendezvous communication is supported by many systems. In such a system, a task (client) sending a message to another task (server) is blocked until it gets a reply from the server, and then it resumes execution in parallel with the server. Examples include Remote Procedure Calls (RPC) in distributed system that can be modeled by a SRVN model. Another example is represented by the large class of transaction processing systems.

An SRVN model includes a set of communicating tasks (see Figure 1.4). A task receiving rendezvous (RV) service requests has a FIFO input queue, where RV requests from clients are 
waiting to be served. At the beginning of its cycle, the server chacks its input queue. If the queue is empty, it waits on the "receive" primitive. If the queue is not empty, the server selects the first request from queue to serve and starts an execution cycle. Afte: a first phise of service, the server sends a reply message back to the client which is blocked waiting for response from the server. Upon receiving the reply, the client is released. The server may then do a second phase of service in parallel with the client, for example some "house-keeping" work which can process in parallel with the client. After the second phase, the server finishes the current cycle and then the server goes back to check its input queue.

Servers may have multiple entries each of which can represent a different behavior with different workload. Hierarchical service is possible to be represented by SRVN model. A server which is serving a client may send nested requests to other servers.

An example of SRVN model from [Pet91] is shown in Figure 1.4. The model represents a

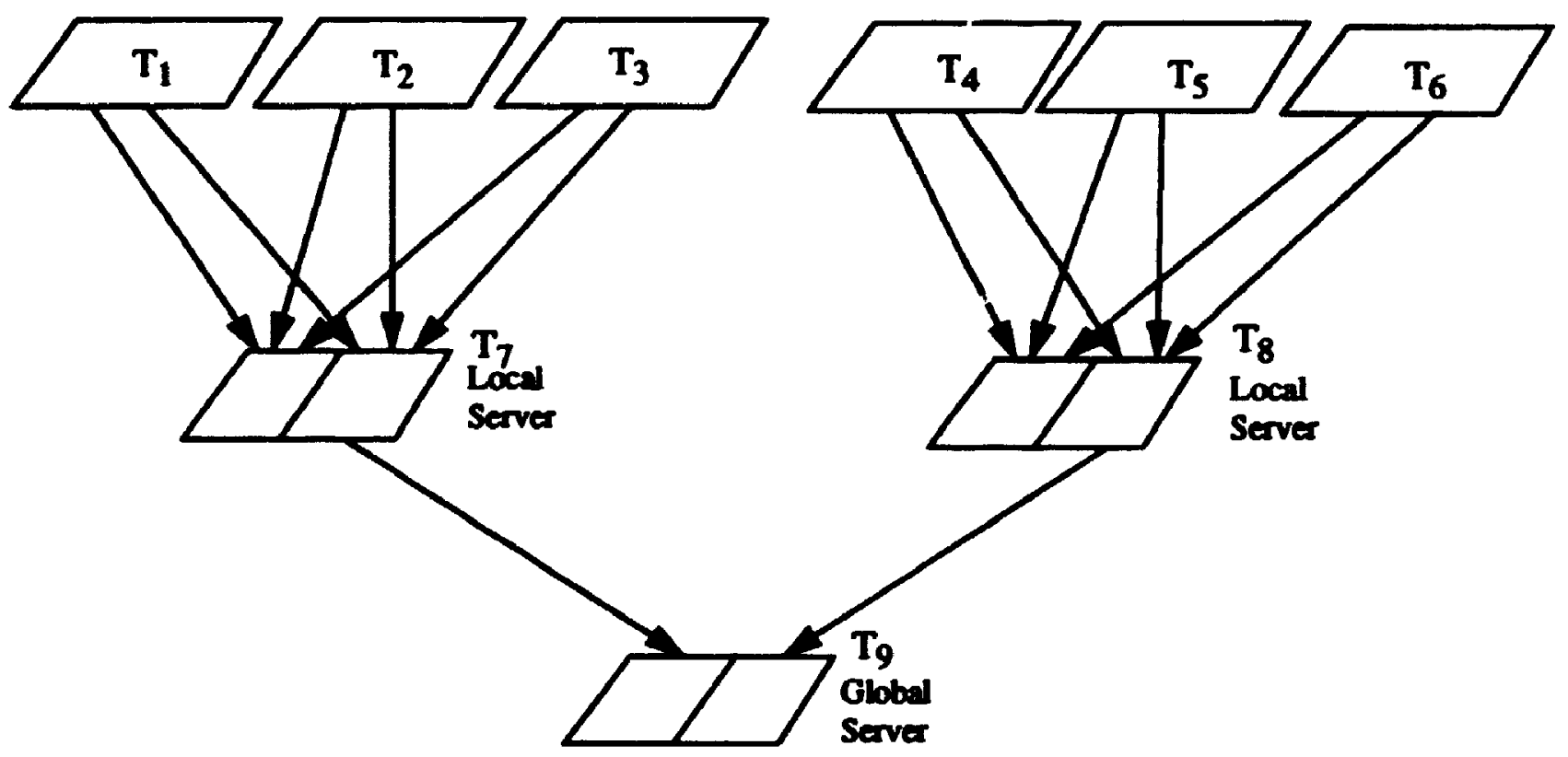

Figure 1.4. Example of SRVN model 
hierarchical database system. There are two kinds of servers: local server and global server. Each local server has its own set of clients. Both local server and global server have multiple entries to offer different service for different request types. Most of the client requests can be satisfied in their local server, only a small proportion of the requests need service also from the global server which is called by local server. While the global server is processing the request, both the local server which sends the request to the global server and the client which initials the request are blocked.

An SRVN model is very effective in modelling concurrent system in which tasks use rendezvous communication. An SRVN captures the following features of a system: nested service offered by the hierarchy of servers, change of request classes by the clients, and parallel execution between clients and servers. However the clients have a single thread of execution and simple behavior.

Rendezvous Networks with Multi-Threaded Client (RNMTC) is an extension of the SRVN model introduced in this thesis, where each client is represent $d$ by a precedence graph and may have a variable number of execution threads. The RNMTC model is presented formally in Chapter 2. The model of a client is a precedence graph composed of a number of nodes and directed edges. The execution flow may consist of multiple concurrent execution threads whose number can vary due to fork and join operations. An execution thread represents an independent flow of control and may request services from servers. The communication between clients and servers is by rendezvous. The servers are similar to those in SRVN models. When a particular thread is blocked in Rendezvous with a server, other threads of the same client may continue their execution, thus enhancing concurrency and performance

The papers known from literature on modelling concurrent software system, which are the closest to our approach are [TB86] and [KME89]. Thomsian and Bay have used hierarchical decomposition method to handle parallel software characterized by a precedence graph [TB86]. Another decomposition technique for modelling concurrent software with looping constructs is introduced by A. Kapelnikov [KME89]. None of these works deals with the synchronous clientserver interaction and the nested service that is aptly captured in the model presented in this thesis.

The important features of the RNMTC model are summarized here: 
- Nested Service: An important feature of the model is its capability of modelling nested service that exists in systems where a software server uses the services of other servers to provide the requested service to the client.

- Modelling Flexibility: Both system software and hardware components can be modelled.

- Multi-Phase Behavior of Servers: The server execution can be characterized by two phases, an in-rendezvous phase and another optional post-rendezvous phase.

- Multiple Multi-threaded Clients: The model can handle multiple application programs each of which may be multi-threaded. Classes of clients are also supported.

The first three features are available in the SRVN model whereas the last one is a new contribution made by the research presented in the thesis.

The analytic solution for the RNMTC model is based on hierarchical decomposition: at the higher level, system behavior is represented by a Markov Chain (MC) model whose states correspond to all possible combinations of client execution states; at the lower level, the system is represented by SRVN models with simple clients, for which existing approximate MVA solution were introduced in several papers, as for example [PW91] [WNPM89] [PW93] [Woo89] and [Pet93]. The last one is incorporated in the algorithm for RNMTC models in this thesis.

Again a number of test cases have been examined. Comparisons between the outputs of the analytic solution and simulation results are made for checking the accuracy of the analytic solution. The results obtained from these comparisons are quite encouraging. The error rate is less than 5\% in most of the cases. The computation time for the model has also been observed to be orders of magnitude smaller than the simulation runs.

This thesis also investigates bounding techniques for the certain types of RNMTC models. Computation of approximate analytic solution is based on a number of assumptions, such as, the exponential distribution of execution time and service time. Bounds have weaker assumptions about the stochastic behavior of the system. For some systems, the distributions for service times and thread execution times may be unknown. The bounds are the only performance measures that can be computed in such a situation. Techniques for the computation of an upper bound and a lower bound on completion time of clients are developed. 
The main result of the thesis constitute a paper [PMLH94] that will be presented at the International Workshop on Modeling, Analysis and Simulation of Computer and Telecommunication Systems, MASCOTS'94, to be held in Durham, North-Carolina, January 31 - February 2, 1994.

\subsection{Outline of Thesis}

This thesis is divided into seven chapters. Chapter 2 describes formally the RNMTC model. Chapter 3 describes a simulator for RNMTC models built on top of the PARASOL simulation kernel [Nei_1]. The simulator is used to assess the accuracy of the approximate analytic solution method and to check the correctness and tightness of bounds for RNMTC models. A guide for using the simulator is presented in Appendix. Chapter 4 introduces the analytic solution method for RNMTC models. The hierarchical decomposition mechanism is discussed. Techniques for the computation of bounds on performance are discussed in chapter 5. The upper bounds and lower bounds for different kinds of system are derived. A discussion of the implementation of the techniques are included through BNR prolog [BNR88] along with some test results. Chapter 6 assesses the accuracy of the analytic solution through many test cases. A set of fork/join test cases and a large model for a On-Line Transaction Processing system are discussed. The purpose of various kinds of test cases is to show that the analytic solution method is applicable to a large range of systems. Chapter 7 presents the conclusions and future work. 


\section{Chapter 2}

\section{RNMTC Model}

The Rendezvous Network with Multi-Threaded Client (RNMTC) model consists of two submodels: the client submodel and the server submodel. The client submodel contains a set of clients, each one represented by a precedence graph which consists of a set of nodes with precedence relationship among them. The server submodel is a set of SRVN-like servers communicating by redenzvous. A server task may receive a request either from a client task or from another server.

Since the RNMTC model is an extension of SRVN model, we review the definition of the latter first.

\subsection{The SRVN Model}

SRVN stands for Stochastic Rendezvous Networks. SRVN is a performance model initially proposed in [Woo89] which can model concurrent networks with rendezous (RV) style of communication.

The SRVN is represented by a "communication graph" (see Figure 2.2) where the nodes are tasks and the edges are RV requests.

There are two types of tasks: neference task and non-reference task. In Figure 2.2, $T_{1}$ and $T_{2}$ are cailed reference tasks whereas other tasks are non-reference tasks. Reference tasks loop endlessly and function as pure clients. During its execution, a reference task may make service requests to non-reference tasks. 
The communication between tasks in SRVN model is by RV. Figure 2.1 shows the behavior of a client and a server over time. After executing on its own CPU, the client makes a request to a server, then it is blocked. The client will resume operation only after receiving a reply from the server. All rendezvous requests are queued in a single FIFO queue at the server. The server accepts the first request from its queue and executes an in- $R V$ phase. During this phase. th server may make nested requests to other servers. After ending the first phase and sending a reply to the client, the server may ente. its post- $R V$ phase which is parallel with the execution of the client. In this second phase, the server executes on its CPU and can make requests to other servers. At the end of the second phase the current cycle is completed. The server starts a new service if there are queue requests or becomes receive-blocked waiting for another request to arrive.

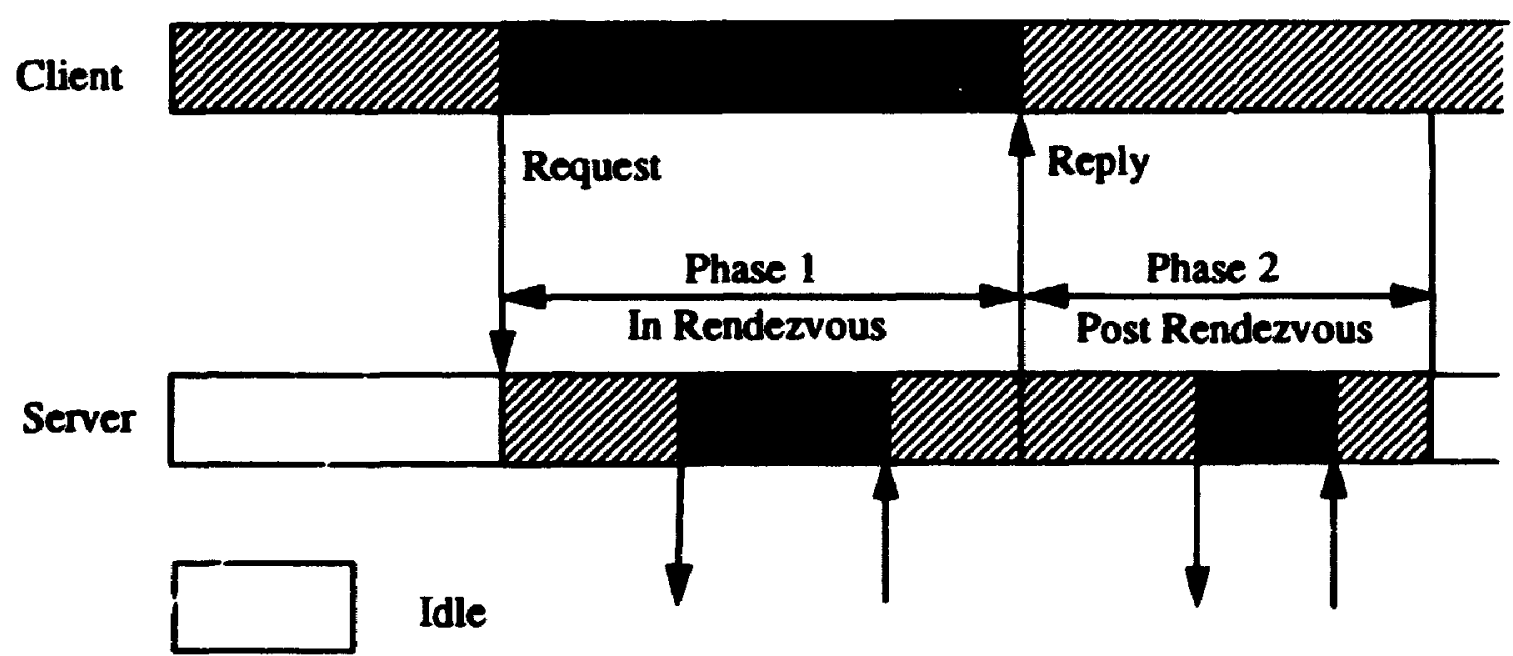

Executing

Rendezvous with other servers (or Blocked)

Figure 2.1. SRVN task behavior

The execution cycle of a non-reference task is characterized by two phases of execution: an "in-rendezvous" phase (phase 1) and a "post-rendezvous" phase (or phase 2). In either phase, a non-reference task may execute on their own and/or request services from other non-reference tasks. The reference tasks only have one phase: phase 2. 
A non-reference task or server may have multiple entries with different workload characteristics. Different requests may arrive at Jiffirent entries of a server according to different service requirements. For example in Figure 2.2, $T_{4}$ has two entries.

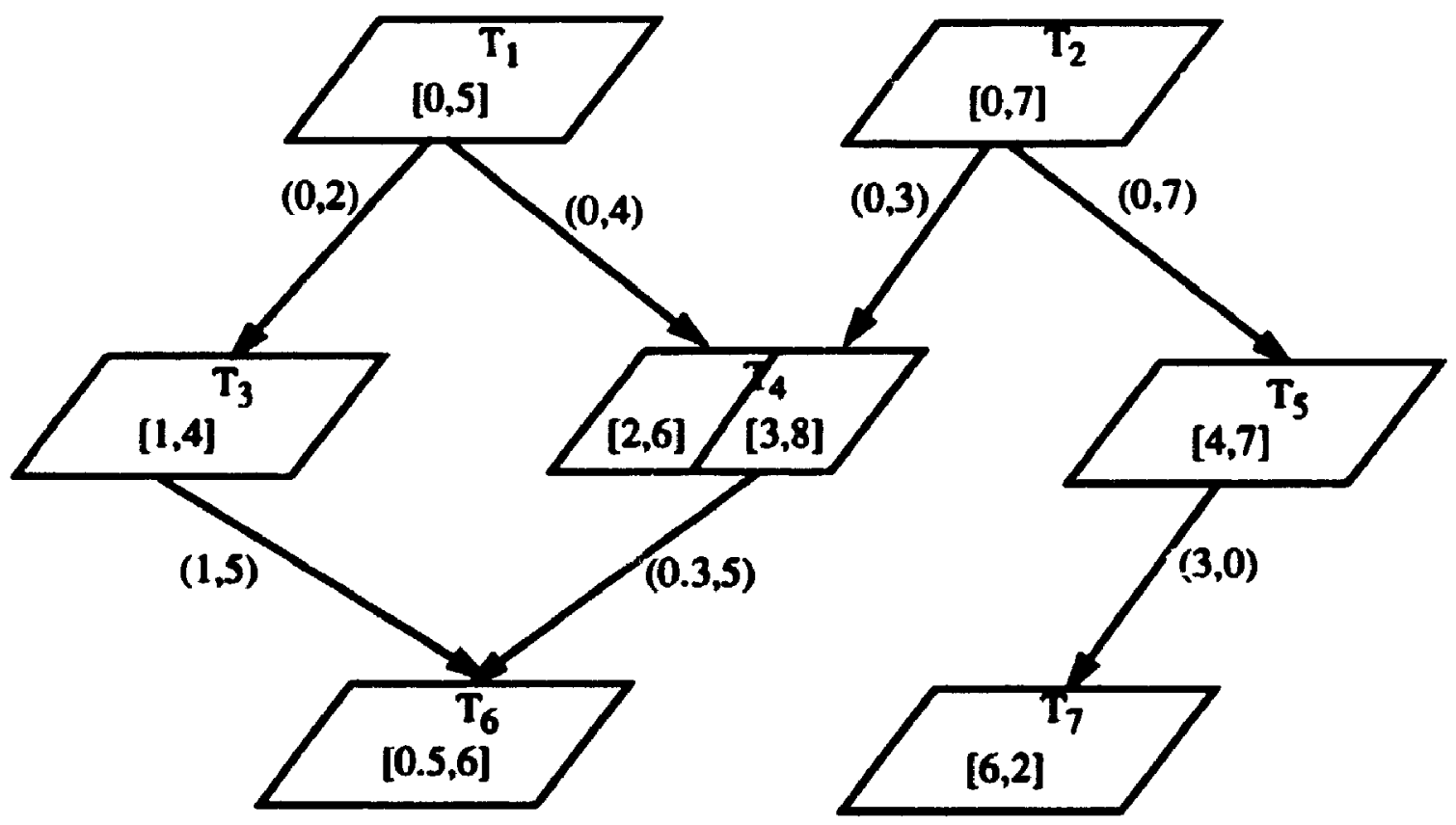

Figure 2.2. Example of an SRVN model

Concurrence is one of the major characteristics in SRVN models. In Figure 2.2, $T_{1}$ and $T_{2}$ are concurrent tasks both of which send service requests to server $T_{4}$, which raises contention for $T_{4}$.

The SRVN model is characterized by the following parameters:

$s(i, e, p)=$ mean computation time of task $i$ in entry $e$ during phase $p ;(p=1$ or 2$)$.

$y(i, e, p, j, d)=$ mean number of RV requests made by task $i$ in entry $e$ during phase $p$ to entry $d$ in task $j$. 
In Figure 2.2, the values in square brackets represent the computation times in the first phase and second phase of the corresponding entry. The values in round brackets on each edges are the mean number of $R V$ requests from one task (entry) to another.

Each task phase is a sequence of identical exponentially distributed stochastic execution periinds, separated by RV requests to other tasks.

On average, an entry $e$ in phase $p$ of task $i$ makes $Y(i, e, p)=\Sigma_{j} \Sigma_{d} y(i, e, p, j, d)$ requests to other tasks in one cycle. The mean duration of an execution period or time slice between two requests is $\mu(i, e, p)^{-1}=s(i, e, p) /(Y(i, e, p)+1)$. After each time slice, the event following every execution period is selected randomly by the task: either makes a request to entry $d$ in task $j$ with probability $y(i, e, p, j, d) /(Y(i, e, p)+1)$ or completes the cycle with probability $1 /(Y(i, e, p)+1)$.

SRVN can model software servers as well as hardware servers(e.g. CPU). If several tasks are collocated on the same CPU, the impact of the processor contention must be modelled. We can model the processor contention by transferring to the processor the execution time of the software tasks collocated on it. Since each task phase may have a different execution time, the SRVN task representing the processor will have several entries.

For example in Figure 2.3, $T_{1}, T_{2}, T_{3}$ and $T_{4}$ are collocated on the same CPU. The CPU is modelled as an SRVN server (called CPU serve)) which has only "in RV" phase and is a pure server due to the fact it never makes requests to other servers. If a task $T_{i}$, entry $e$ in phase $p$ makes $Y(i, e, p)$ requests to other non-reference tasks, it will perform $Y(i, e, p)+1$ computation periods during the current phase. Therefore the mean visit ratio from $T_{\mathrm{i}}$ to $C P U$ server is $Y(i, e, p)+1$. If the computation time $s(i, e, p)$ is transferred to entry $d$ in the CPU server, then the service time of entry $e$ takes the value $s(i, e, p) /(Y(i, e, p)+1)$.

An example is shown in Figure 2.4 which is the SRVN model for Figure 2.3. $T_{3}$ has totally 1.5 mean requests to software servers $\left(T_{2}\right.$ and $\left.T_{4}\right)$. It performs 2.5 computations one cycle in average. Its computation time is transferred to entry 3 in CPU server. Time slice in entry 3 is $3 / 2.5=$ 1.2 .

By modelling a hardware resource as an SRVN server, hardware performance measures, such as utilization, queue length, can be obtained by solving the SRVN model. 


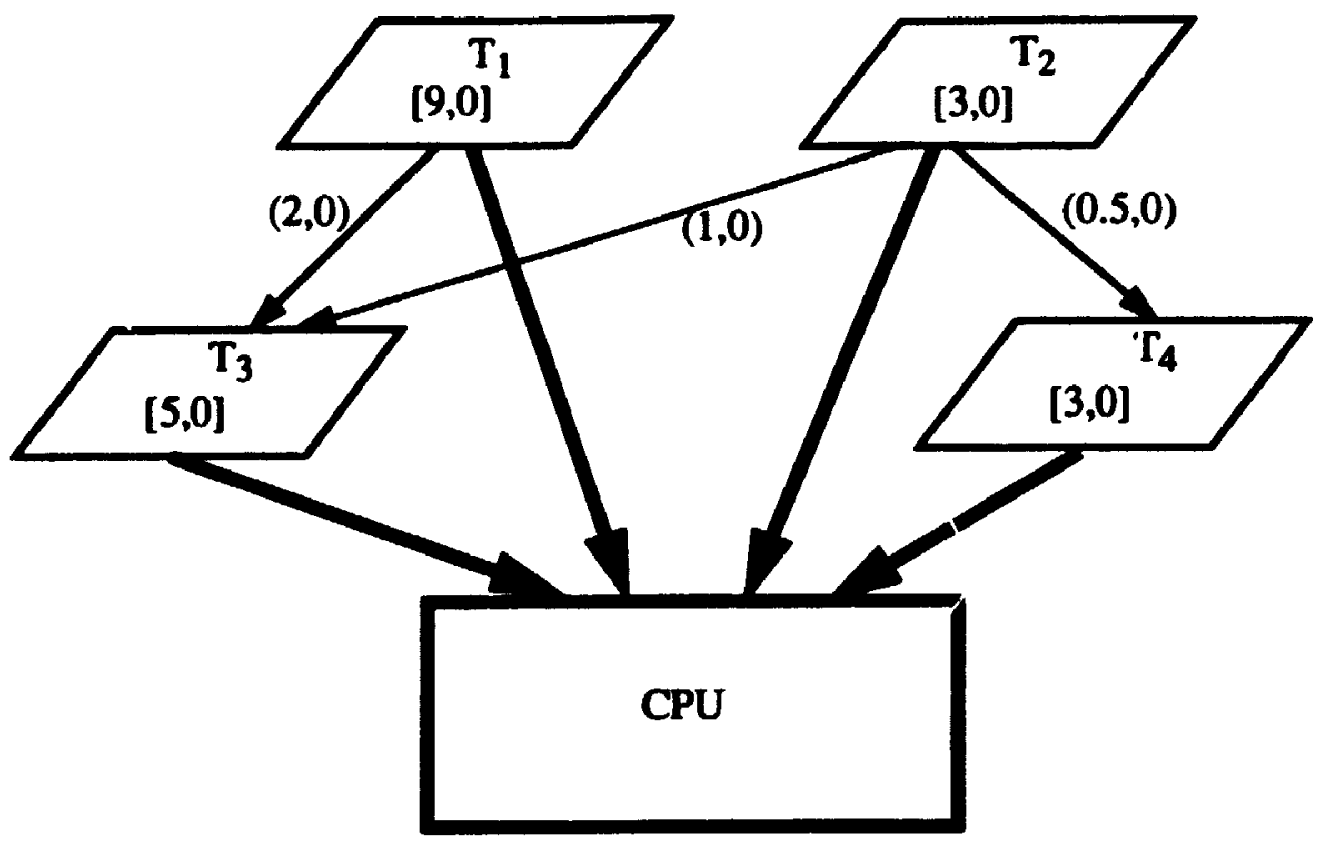

Figure 2.3. Tasks collocated on one CPU

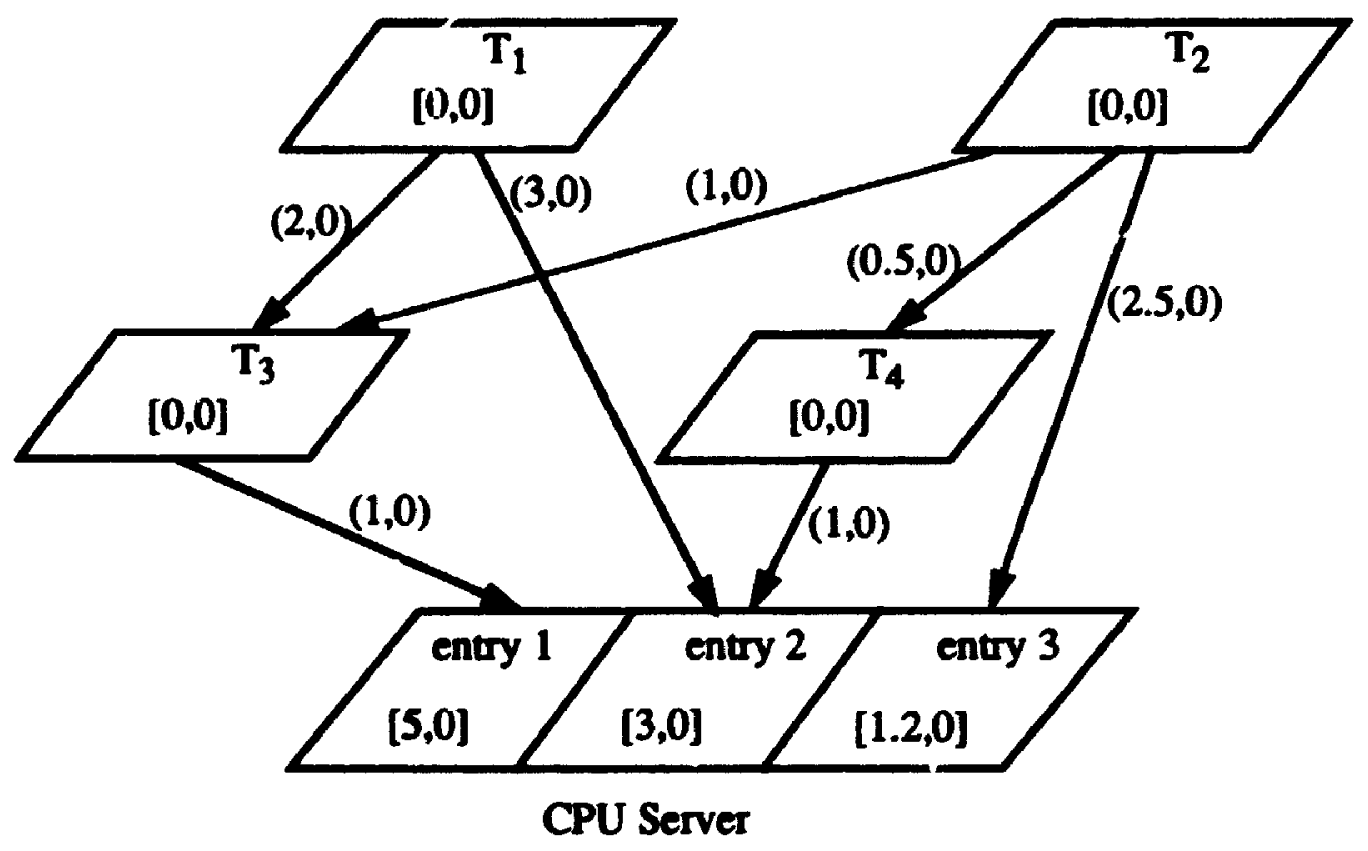

Figure 2.4. SRVN model for processor contention 


\subsection{Definition of the RNMTC model}

As mentioned before, an RNMTC model consists of a set of complex clients (each ciescribed by a precedence graph) which are requesting services from a set of SRVN-like servers. If several clients are described by similar precedence graphs and request the same servers with the same mean visit numbers, they can be grouped in a "class of clients", as will be shown in section 2.2.2.

\subsubsection{Client Precedence Graph}

A precedence graph is a collection of nodes and directed edges. Each node represents the execution of a program component. Node execution time is the sum of node computation time, service time at servers and queueing delay due to contention for servers. Computation time is the time that a node consumes on its own. The edges represent precedence relationship among nodes. A node is called a source node of edge $k$ if the node is the origin of the edge $k$ (e.g. node $i$ in Figure 2.5). A node is called destination node of edge $k$ on which edge $k$ terminates (e.g. node $j$ in Figure 2.5).

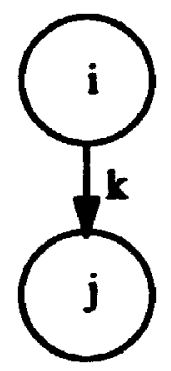

Figure 2.5. Simple precedence graph

The end of the edge with an arrow is called the head of the edge while the opposite end of edge is called the tail of the edge.

For the edges terminating on a single node, there are two basic relationships: AND and OR. If the edges do not have any symbols, the destination node can not be active until all the source nodes connected with the edges are finished. This is an AND relationship (Figure 2.6 a). If gyinivol " + " appears on the head of the edges, the destination node can be initiated after any one of the 
source nodes completes its execution. This is called an OR relationship (Figure $2.6 \mathrm{~b}$ ). In order to represent more complex precedence relationships, edges can be grouped hierarchically by drawing ellipses around them (Figure $2.6 \mathrm{c}$ ). In Figure $2.6 \mathrm{c}$, the edges included in the UNION (in ellipses) are connected by OR, and the result of the UNION and the remaining edges are connected by AND.

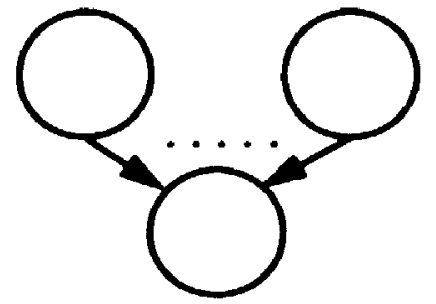

AND

(a)

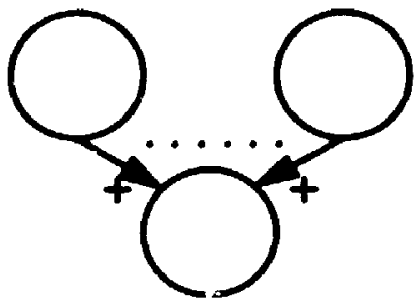

OR

(b)

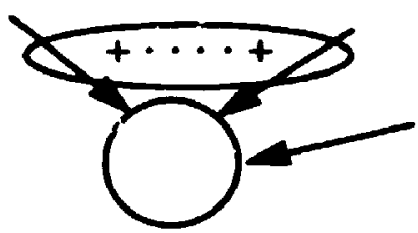

UNION

(c)

Figure 2.6. Edge terminating at the same node

For the edge emanating from a single node, there are also two basic relationships: AND and EXCLUSIVE-OR. If there are no symbols on the edges, all the destination nodes are to be activated after the source node is completed. This is an AND relationship (Figure 2.7 a). For the EXCLUSIVE-OR case, a probability $p_{i}$ is associated with each edge. After the source node completes, only one of the destination nodes is activated. Destination nodes $1, \ldots, n$ are initiated with the probabilities $p_{1} \ldots p_{n}$ (Figure 2.7 b). Edges emanating from the same node can also be combined hierarchically by applying the UNION relationship (Figure $2.7 \mathrm{c}$ ). For example, in Figure $2.7 \mathrm{c}$, the edges included in the UNION are connected by AND, and the UNION result and the remain- 
ing edges are connected by OR.

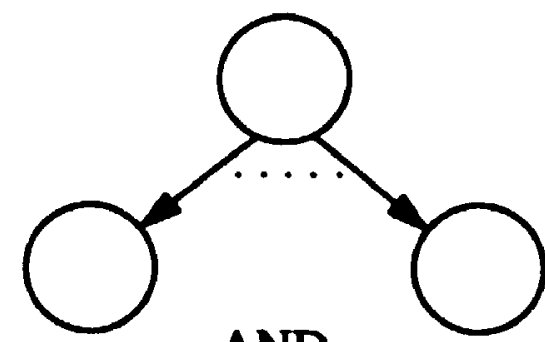

AND

(a)

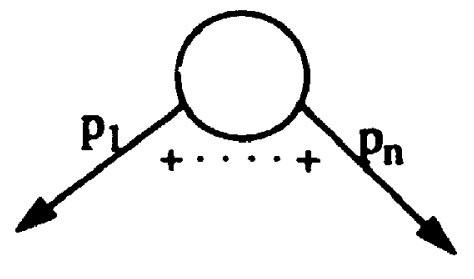

Exclusive-OR

(b)

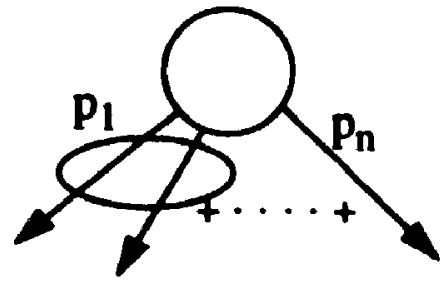

UNION

(c)

Figure 2.7. edge emanating from the same node

There is also another kind of relationship called LOOP that may exist in a precedence graph (Figure 2.8). After node $i$ is finished, either node $k$ may be active with probability $l$ - $p$ or node $j$ is initiated again with loop probability $p$. Inside the loop any graph structure is allowed.

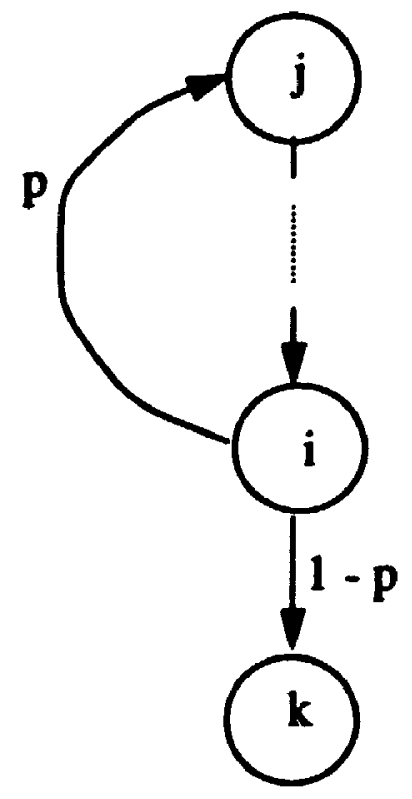

Figure 2.8. Loop relationship

A precedence graph describing an RNMTC client may include some or all of the basic rela- 
tionships presented above. Each RNMTC client is executed cyclically. The execution of a client is characterized by a number of different states. Each state corresponds to a set of particular tasks (nodes) that are active concurrently. In Figure 2.9 (a), are shown two simple graphs to give examples of single-threaded and multi-threaded execution states. The client in Figure 2.9 (a) has three execution states $\left\{a_{1}, a_{2}, a_{3}\right\}$, all of them single-threaded. On the other hand, Figure 2.9 (b) expresses an AND relationship. After node $b_{1}$ finishes execution, the execution thread is forked to two parallel branches and then join them before starting a new cycle. The client in Figure 2.9 (b) has four execution states $\left\{b_{1}, b_{2} b_{3}, b_{2}, b_{3}\right\}$, only the first state $b_{1}$ is single-threaded, while the last three states are multi-threaded, since the last three occur after a fork but before the corresponding join.

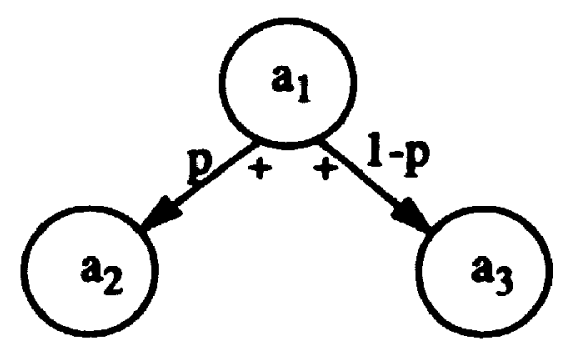

(a)

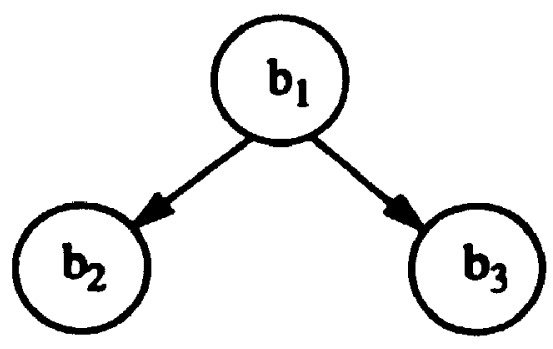

(b)

Figure 2.9. Simple precedence graph model

A more complex example of a client graph that executes cyclically is given in Figure 2.10 in which multiple relationships are included. And the loop may also exist. After the termination of a node, the operation routine may loop back with probability $p_{1}$, or invoke its successor with probability $1-p_{l}$.

In a precedence graph, a node which has a successor node but has no predecessor node is defined as an initialization node, a node which has a predecessor node but has no successor node is called termination mode, and a node which has both a predecessor node and a successor node is named middle node. Node 1 in Figure 2.10 is a initialization node, Node 8 is a termination node 
and the rest are middle nodes. Another special node is called isolation node which has neither a predecessor nor a successor node.

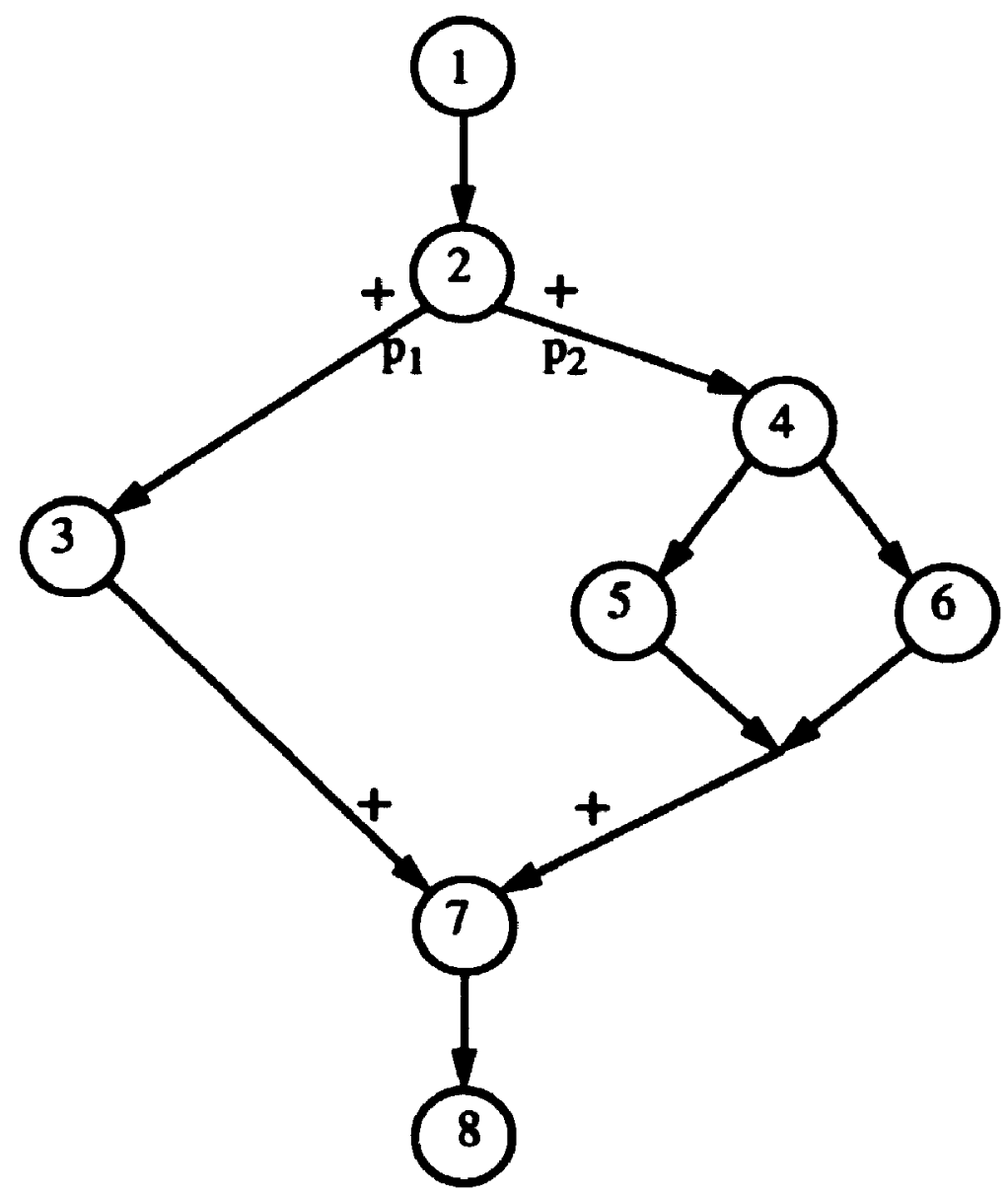

Figure 2.10. Precedence graph model

\subsubsection{RNMTC Model Parameters}

As stated before, an RNMTC model contains a set of clients and a set of servers. Each node in a client precedence graph is called client task. Figure 2.11 shows a simple RNMTC model with one client. During the execution of the client according to its precedence graph, some of client tasks may make service requests to servers. The communication between a client task and a server is by RV. When a client task is being served by a server, the client task is blocked until the server sends a reply message. A client task computes for a time, and then either decides to send another request to a server or to terminate itself, activating its successor(s). After the execution of the ter- 
mination node, a new cycle will be started.

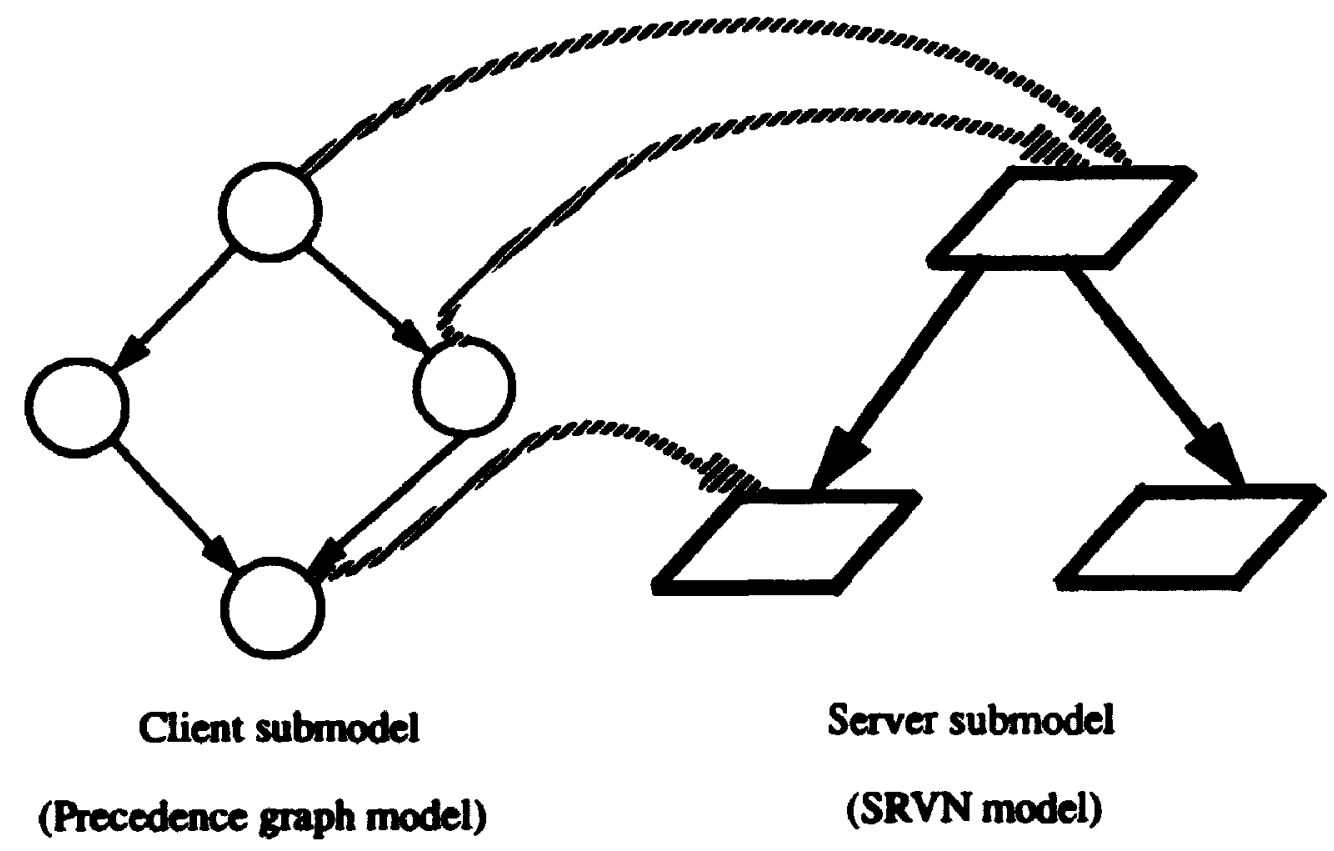

Figure 2.11. Simple RNMTC model

Figure 2.12 illustrates an RNMTC model with multiple clients.

Each client is described by an independent precedence graph. All clients execute concurrently, sharing the same set of servers. The contention for servers exists not only within a client but also among different clients. If several clients are described by identical precedence graphs 
and they request exactly the same servers, then the clients can be grouped in the same "class"..

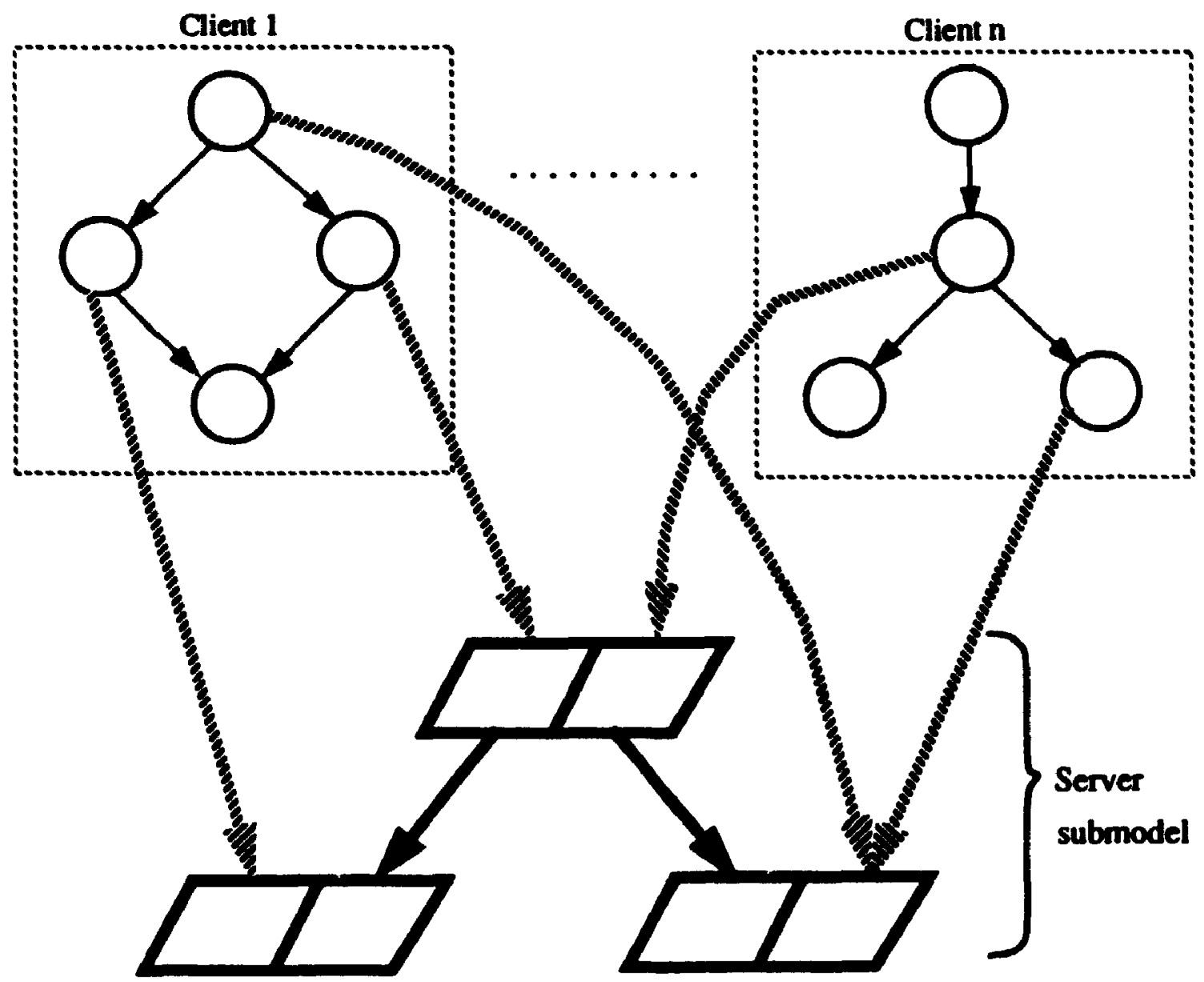

Figure 2.12. Multiple client RNMTC model

The parameters of an RNMTC model are the computation times and mean visit numbers of client tasks to servers and the service times and mean visit numbers of servers to other servers. The following notations will be used for RNMTC model parameters.

$C_{a}=$ total number of classes in the client system

$C_{(}(i)=$ total number of clients in class $i$

$N(i)=$ total number of client tasks in one client of class $i$

$K=$ total number of servers in the system 
$U(j)=$ total number of entries in server $j$

$c(i, t)=$ average computation time for client task $t$ in class $i$ per job cycle

$s(i, e, p)=$ average service time in entry $e$ during phase $p(p=1$ or $p=2)$ of server $i$ per visit

$S(i, e)=$ average service time in entry $e$ of server $i$ in two phases per visit

$$
S(i, e)=s(i, e, l)+s(i, e, 2)
$$

$v(i, t, j, u(j))=$ average number of visits made by client task $t$ in class $i$ to entry $u$ of server $j$ per job cycle

$v(i, t, j)=$ average number of visits made by client task $t$ in class $i$ to server $j$ per job cycle

$$
v(i, t, j)=\sum_{u=1}^{U(j)} v(i, t, j, u(j))
$$

$y(i, e, p, j, d)=$ mean number of RV requests made by server task $i$ in entry $e$ during phase $p$ to entry $d$ in server task $j$.

The principal performance measures for an RNMTC model are the cycle time for each of its clients. All other measures (throughputs, server utilizations, etc.) can be derived from client cycle times and model parameters. 


\section{Chapter 3}

\section{RNMTC Simulator}

\subsection{Motivation for Simulation}

Simulation is an important tool for performance evaluation. Simulation involves constructing a model for the behavior of a system processing an appropriate abstraction of the workload. The major advantage of simulation is its generality and flexibility. A number of important issues must be considered in simulation.

.. It must be decided what to simulate and what not to simulate and at what level of detail. Simply duplicating the detailed behavior of the system is usually unnecessary and prohibitively expensive.

2. A careful experimental design is essential to keep the simulation cost down.

Simulation is frequently used to examine the accuracy of an analytic approximate algorithm such that the modeler has an idea if the accuracy of the algorithm is in acceptable limits. This is the main motivations for building the RNMTC simulator in this thesis. The analytic results of the approximate method for RNMTC models introduced in Chapter 4 will be compared with simulation results for a large number of test cases in Chapter 6. Another use of the RNMTC simulator is to assess the correctness of the upper bounds and lower bounds for certain classes of RNMTC models studied in this thesis.

The RNMTC simulator is built on top of a distributed system simulator kemel called PARASOL, which is briefly presented in the next section. 


\subsection{Brief Introduction to PARASOL}

PARASOL is a simulation tool developed at the School of Computer Science, Carleton University. As a simulator, PARASOL allows the user to define the description and the behavior of both hardware and software for distributed and/or parallel computer systems. It can be used to predict the performance of the distributed/parallel systems or as a prototyping tool. The detail description of PARASOL simulator system can be found in [Nei_1] and [Nei_2] and this section gives only a brief introduction.

- Organization

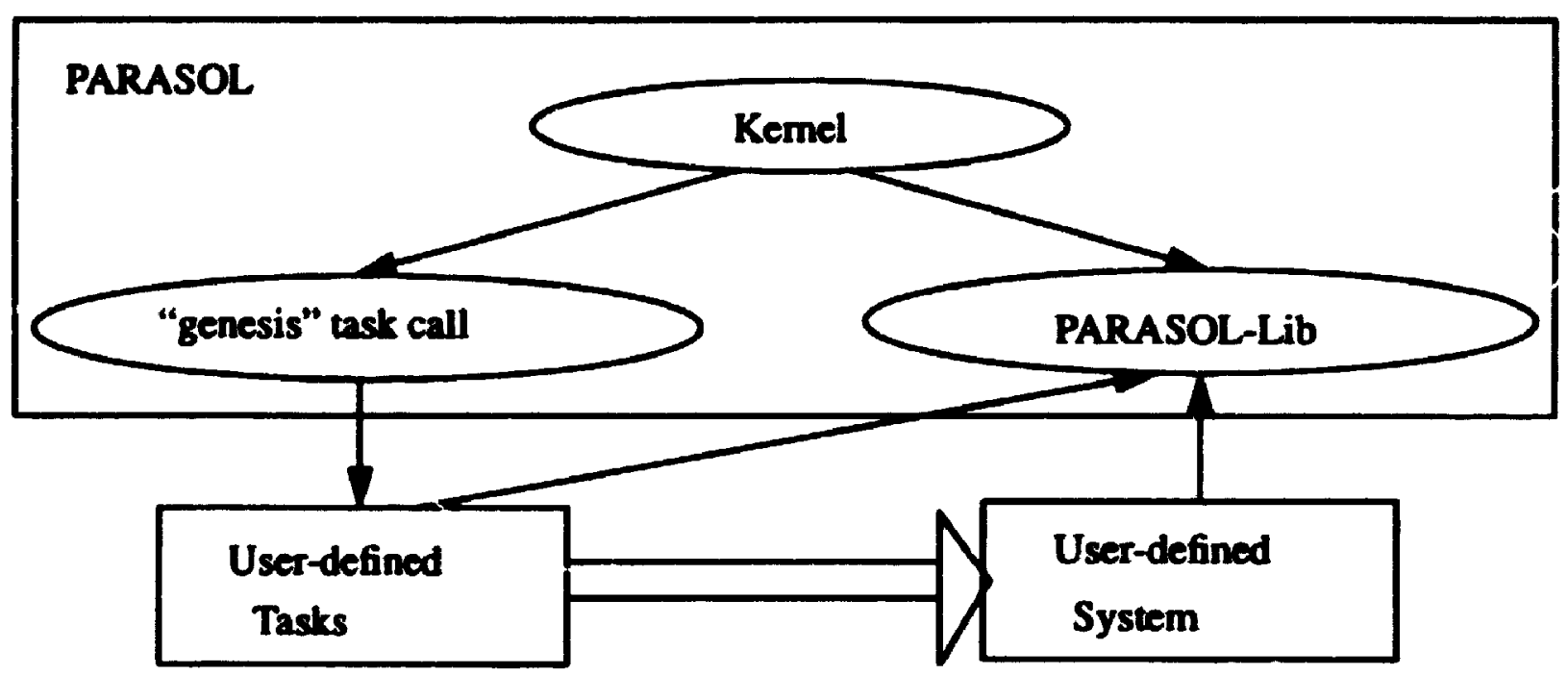

Figure 3.1.PARASOL organization

Figure 3.1 shows the logical organizaton of the PARASOL simulator. The kernel is the implementation of PARASOL simulator and written entirely in C programming language. It supports two user interfaces: genesis tasks and PARASOL library. Genesis is a standard, PARASOL system defined task interface which is called automatically when the PARASOL system is started. This is the first task which allows the user to define the simulated hardware specification and the initial configuration of the system with respect to simulated application tasks.

The PARASOL library contains the functions called by the user defined tasts and the hardware specification. When the system is running, it will simulate the behaviors of user defined 
tasks running on the user described hardware system.

\section{Handware Specification}

The PARASOL can simulate a set of hardware resources, such as CPUs, buses and links between different hardware components.

The PARASOL supplies a set of methods of how to define the simulated system hardware. This can be done by "genesis" task by means of calling three functions: build_node, build_bus and build_link. Nodes must be (onstructed before the construction of buses and links.

\section{Task Management and Communication}

After the hardware is built, the tasks will be assigned on the system. The tasks can model either hardware or software components. It is the responsibility of the user to create. activate and manage all the tasks except one task - genesis which is managed by PARASOL.

The PARASOL supplies the methods of creating, resuming, killing and suspending tasks. The tasks can sleep, be awakened and migrated. The tasks can communicate with each other by sending/receiving message and broadcasting.

\section{Statistic Report}

In order to obtain the desired performance measures, PARASOL provides for the automatic capture of hardware device utilization statistics by means of the stat_flag arguments in "build" instructions. To meet user's requirement, PARASOL also supplies a mechanism for two types of user defined statistical data. The first is "TIME" used to capture device utilization statistics and the second is "POP" used for population statistic, such as mean response time, mean waiting time, etc.

\subsection{Architecture and Implementation of the RNMTC Simulator}

The RNMTC simulator is a discrete events simulator. The RNMTC simulator gives the abstract simulation results when analytic solution is discussed. 


\section{Function of RNMTC Simulator}

The RNMTC model may have multiple classes of client and each class may contain multiple clients. Each client is described by a precedence graph which may have the following task (node) types: initialization task, middle task, termination task and isolation task.

A client precedence graph may include the following types of precedence relationships, as described in section 2.2.1: AND, OR, Exclusive-OR, UNION and LOOP.

The RV communication between tasks is also simulated. The servers can have multiple entries and multiple phases.

\section{Implementation of RNMTC Simulator}

Each client has its own "manager" PARASOL task which does not consume any CPU time. Each "manager" has the following responsibilities:

(a) Activate the tasks belonging to the client which the "manager" is in charge of. The manager sends a message to all "initialization tasks" of this client to start an instance of the simulation representing a client in RNMTC system.

(b) Record the job performance measures after the "manager" receives the completion message from all its termination tasks.

In order to reduce the storage requirements for data structures, the redundance of information is reasonably reduced as much as possible. Each individual task has its own identifier including task-class, task-client, task-name and task-id. The last one is assigned by the PARASOL kernel, whereas i'e first three are defined by user. However, the same parameters for each task in the same class, such as mean computation time, mean visit ratio to servers, are stored only once. Different clients in a class share the parameter data.

After the simula $: n$ is activated by genesis, each "manager" task starts activating and controlling its own subsystem. By looking at the task identifier with the same class and client, the "manager" can find its client tasks and get the task parameters from a data record.

The data specification for the simulated system is inputted by a program named "datainput" 
and RNMTC simulator reads data and runs the simulation according to the data specification.

The RNMTC simulator was extensively used to analyze different test case that will be presented in Chapter 5 and Chapter 6.

Instructions for running the RNMTC simulator can be found in Appendix. 


\section{Chapter 4}

\section{Approximate Analytic Solution of RNMTC}

\subsection{Solution Introduction}

The solution for RNMTC models introduced in this chapter is based on hierarchical decomposition: at the higher level, the system behavior is represented by a Markov Chain (MC) model whose states corresponding to all possible combinations of clients executions states; at the lower level, an SRVN with simple clients corresponds to each MC state.

Several MVA approximate solutions for SRVN were previously introduced. The solution presented in [PW91] and [Pet93] was used here.

Various decomposition methods are knuwn for the analysis of concurrent software systems described by precedence graphs. For example, [TB86] introduces a algorithm which uses a decomposition method to obtain the cycle time of a tasking system modelled by a simple precedence graph without loop or probabilistic branching. Also in [KME89] is introduced a modelling methodology for the analysis of distributed software modelled by a complex precedence graph with loops and branching running on a hardware system modelled by QNs. The approach presented in this thesis is similar to that in [TB86] and [KME89] with the following differences:

- In our case the high level model represents a set of independent clients that may belong to several classes each one described by a precedence graph, instead of a single instance of a tasking system modelled by a single precedence graph.

- The lower level models are SRVNs instead of QNs, where the servers act also possibly as 
clients to other servers (allowing for multi-layered service), and the service can be offered in two distinct phases (one "in RV" while the server is processing requests from a client task and the client task is blocked and the second "post RV" which is active in parallel with the client task).

The model of RNMTC is solved by hierarchical decomposition: at the higher level, the system behavior is represented by a Markov Chain (MC) model and at the lower level, the system is represented by SRVN models as described in the f.ullowing subsections.

\subsection{Higher Level by MC Model}

MC can represent the behavior of a number of client systems. The states of MC correspond to all possible combinations of client execution states expressed in terms of enabled graph nodes. The transition among states are determined as a function of precedence relationships among the states. The transition rates $\mu$ among the states of the $M C$ are determined from the throughput of the system in processing various combinations of client tasks. Figure 4.1 is a single client subsystem with node 1 and node 2 execute in parallel and then join in node 3.

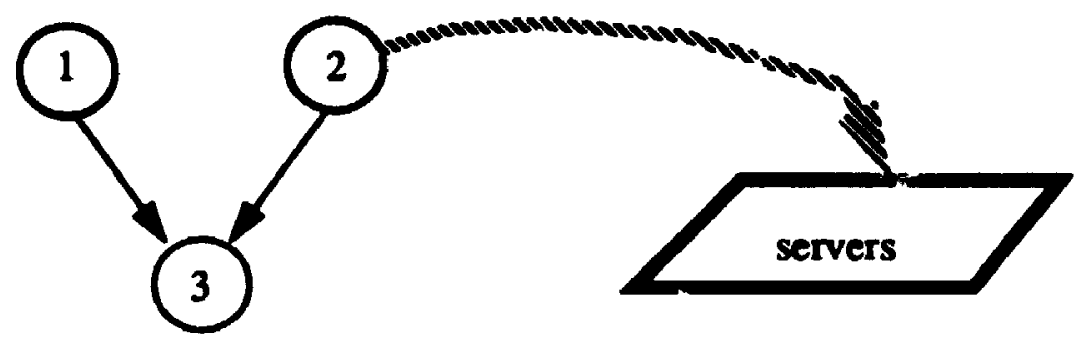

Figure 4.1. A single client system

The MC corresponding to the execution of the system is given in Figure 4.2. In this simple case, the MC is acyclic, except for the final transition from the completion state to the initial state. This implies the repetitive execution of the client system. States $S_{1}, S_{2}$ and $S_{3}$ are multi-threaded since they occur after a fork but before the corresponding join, whereas state $S_{4}$ is single-threaded.

If a system consists of multiple classes, MC becomes more complicated. Figure 4.3 is one example of such a system. Class 1 has 2 clients while class 2 has only one client. The MC graph 


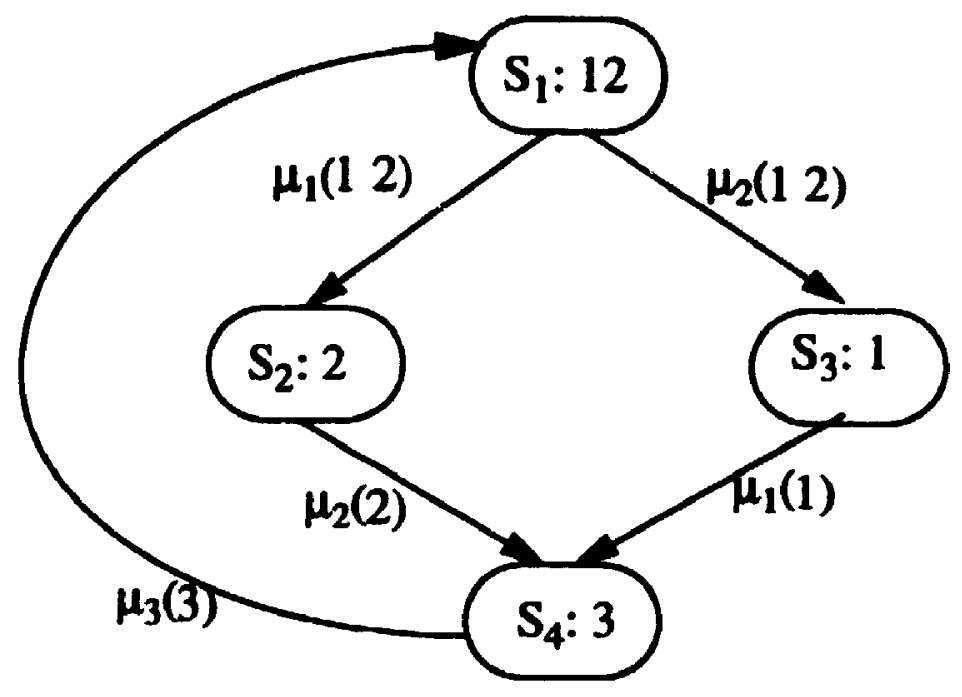

Figure 4.2. MC for simple system

for this system is shown in Figure 4.4.

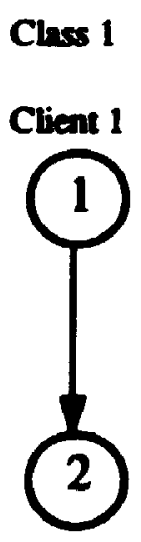

Class 1

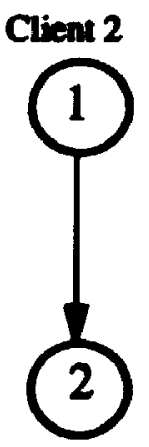

Class 2

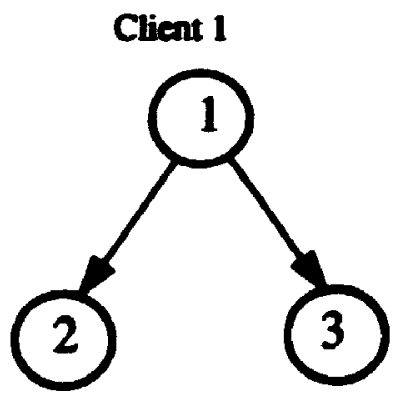

Figure 4.3. Precedence graph with multiple classes 


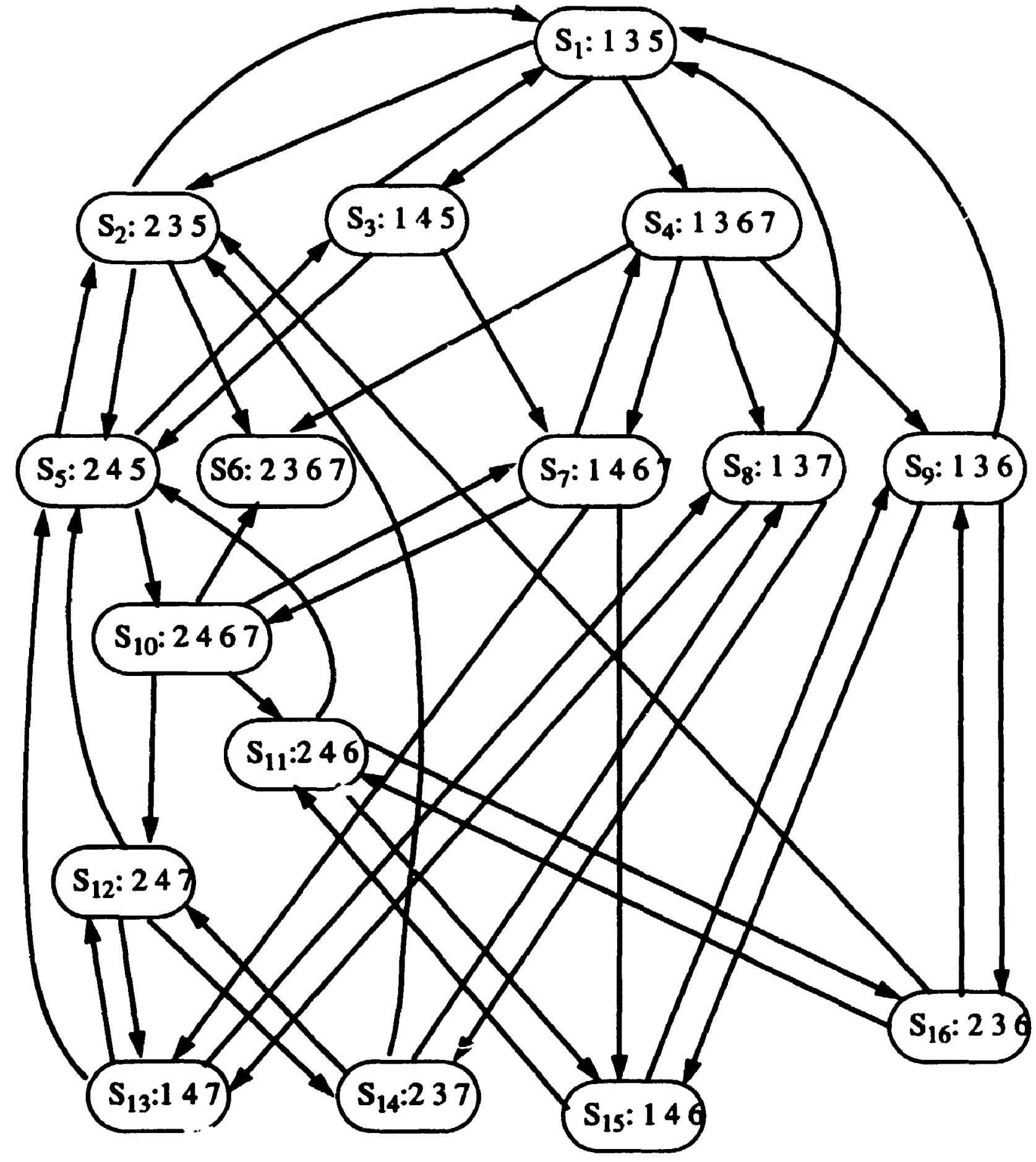

Figure 4.4. MC for multiple client system

In Figure 4.4, the following notations are used. Let $C T(i, j, k)$ be the client task (node) $k$ in 
client $j$ of class $i$. Thus, for example, client task 3 of client 1 from class 2, in Figure 4.3, can be denoted as CT $(2,1,3)$. Each MC state includes a number of client tasks which may be members of different classes and/or different clients. Figure 4.4 shows the MC corresponding to these concurrent clients shown in Figure 4.3. In the MC figure, the client tasks have been renumbered as follows:

$$
\begin{aligned}
& 1=\operatorname{CT}(1,1,1), 2=\operatorname{CT}(1,1,2), 3=\operatorname{CT}(1,2,1), 4=\operatorname{CT}(1,2,2), \\
& 5=\operatorname{CT}(2,1,1), 6=\operatorname{CT}(2,1,2), 7=\operatorname{CT}(2,1,3)
\end{aligned}
$$

There are a lot of cyclic arcs among different states due to the fact that individual client runs independently. The client tasks in each state may compete for servers. The queueing delay due to contention for servers is obtained from the lower level model.

\subsection{Lower level by SRVN Model}

At the lower level, each MC state can be viewed as an SRVN model in which the client tasks active in that state are reference tasks in SRVN, while the RNMTC servers are non-reference tasks in SRVN. The servers are called directly or indirectly by the client tasks in this state.

A few approximate MVA solution methods for SRVN models with simple clients were introduced in [MWNP88] [MWNP89] [PW91] [WNMP88] [WNPM89] and [Woo89] for various classes of SRVN models. Each method solves iteratively a set of nonlinear equations relating tasks and entry throughputs, mean duration of phases and mean waiting for queueing and for service of the RV requests. These methods take a common MVA-like approach, but differ in the way they compute the queveing delays. In the algorithm for RNMTC model is embedded the SRVN method presented in [Pet93]. The main MVA equations are reviewed below.

In SRVN terminology, both the client tasks and the servers are named tasks. The client tasks are reference tasks, and their throughputs represent the main performance results of the model. For notation consistency it is assumed that a reference task has one entry with a single "post RV" phase.

The following introduction referenced from [PW91] and [Pet91] gives the basic procedure to compute performance measures for SRVN system. 
The mean duration of phase $p$ of task $i$, entry $e$, including the waiting for RV requests, is given sxactly by:

$$
x(i, e, p)=s(i, e, p)+\sum_{j} \sum_{d \in E^{\prime}(j)} y(i, e, p, j, d)(w(i, j, d)+x(j, d, l))
$$

where $E(j)$ is the set of entries of task $j$, and $w(i, j, d)$ is the queueing delay suffered by a RV request from task $i$, entry $e$, phase $p$ to task $j$ entry $d$.

The throughput $f_{r}$ of a reference task $r$ (where $r=1, R$ ) is given by the following exact equation, due to the fact that, by definition, a reference task has one entry with a second phase only:

$$
f_{r}=1 / x(r, l, 2)
$$

The throughput $f_{j}$ of a non-reference task is given as the sum of its entry throughputs $\varphi_{j d}$ :

$$
f_{j}=\sum_{d \in E(j)} \varphi_{j d}
$$

The entry throughput $\varphi_{j d}$ of a non-reference task $j$ is related to the throughputs of its clients by the synchronization imposed by RV, as show it in the following traffic equations:

$$
\varphi_{j d}=\sum_{i \in \in \in(i)} \sum_{p} y(i, e p j, d) \varphi_{i e,} j=R+1, N_{i} d \in \mathrm{E}(\mathrm{j})
$$

By solving the set of equations (4-4), the non-reference throughputs can be obtained directly as a linear combinition of the reference throughputs, in the form:

$$
\varphi_{j d}=\sum_{r=1}^{R} a_{j d r} f_{r} j=R+1, N
$$

The queueing delay $w(i, j, d)$ suffered by $i$ at server $j$ for entry $d$ due to other requests (from task $k$ to entry $g$ ) found in service in phase 1 or 2 , or in queve is computed as:

$$
\begin{aligned}
w(i, j, d)= & \sum_{k=18 \in E_{(j)}} \sum_{i j d}^{k g 1}\left(v(j, g, 1+x(j, g, 2))+A_{i j d}^{k, 2} v(j, g, 2)\right. \\
& +B_{i j d}^{k g}(x(j, g, I)+x(j, g, 2))
\end{aligned}
$$


where $v_{j g p}$ is the mean residual life of server $j$, entry $g$, phase $p$, and $A$ and $B$ are the arrivalinstant probabilities defined for a system in steady-state as follows:

$A_{i j d}^{k g p}=$ the probability that a RV request from $i$ arriving to task $j$ for entry $d$ finds $j$ busy executing entry $\&$ phase $p$ to serve a request from another task $k$

$B_{i j d}^{k g}=$ the probability that a RV request from $i$ arriving to $j$ for entry $d$ finds in queue a request from other task $k$ to entry $d$ of $j$

The SRVN algorithm repeats the following procedure, until reaching convergence. First consider that phase durations $x$ and throughputs $f$ are known, and compute new values for the arrival instant probabilities and for the waiting $w$ (4-6). Then, by using the new waiting, new values are determined for phase durations from (4-1) and for throughputs from (4-2), (4-5) and (4-3).

\subsection{Decomposition Method}

The solution for RNMTC model is based on the following assumptions:.

- All execution time of client tasks and service time of servers are exponentially distributed.

- The server queue has a FIFO discipline to serve its clients.

The analytic method obtains an approximate, but reasonably accurate performance results for RNMTC model. The approximate analytic solution for RNMTC proposed in this research proceeds according to the following steps:

a) Construct the high level MC model based on the precedence graph. Each state in MC represents combinations of client execution states.

b) Determine transition rates $\mu$ from the throughputs corresponding to each client task in the state and derive the transition rate matrix $Q=[\mu]$.

- If no requests are made to servers by any of client task in the state, the transition rate is the inverse of the execution time for each client task. For example, in Figure 4.1, client task 2 calls servers while other client tasks do not. The transition rate $\mu_{1}(1)$ for client task 1 in state $s_{3}$ (Figure 4.2) is the inverse of client task 1 execution time. 
- If some or all client tasks call servers, get the transition rate as reference task throughputs obtained by solving the SRVN model. For example, in Figure 4.2, in order to get the transition rates for client tasks in state $s_{1}$, the SRVN solver is called to derive the throughputs for each client task in the state.

c) Build the $M C$ balance equation $Y Q=0$, where $Y$ is a set of state probabilities $p_{s}(i)$. Solve the system of linear equations to get $p_{s}(i)$ for state $i$.

d) Compute the desired performance measures for the client system (as described in section 4.5).

The usage of RNMTC solver can be found in Appendix.

\subsection{Performance Measures}

Different performance measures can be obtained for single client RNMTC system and multiple client RNMTC system respectively. The following sections present the output from the analytic solution method.

\subsubsection{Performance Measures for Single Client RNMTC System}

In Single client RNMTC system, the overall performance and the performance on individual state and task can be computed as follows:

- Cycle time for the client $T_{c}$

Let $\Sigma_{\mu}(1)$ be the total outgoing transition rate from state 1 in $M C, p_{s}(1)$ be state probability in state $1, p_{1}(s)$ is the loop probability from state $s$ to state 1 caused by the lcop construct in precedence graph. Thus, according to the flow balance theory,

$$
T_{c}=1 /\left(\left(p_{s}(1) \sum \mu(1)\right) \cdot \prod_{i=2}^{s}\left(1 /\left(1-p_{1}(i)\right)\right)\right.
$$

where $S$ is the total number of states in MC.

- System throughput $X$ 
$X=1 / T_{c}$

- State $i$ active time $S(i)$

$S(i)$ is the time spent on state $i$

$$
S(i)=T_{c} p_{s}(i)
$$

- Parallelism distribution $P_{p}(n)$

$P_{p}(n)$ is a probability that $n$ tasks are active simultaneously during the ciient execution.

Clearly, $\sum_{i=1}^{S} p_{s}(i)=1$. Therefore.

$$
P_{p}(n)=\sum_{i \in k} P_{s}(i)
$$

Where $R$ is the set of states in which the number of concurrent tasks is $n$.

- Average parallelism Ap

Average parallelism $A_{p}$ is the average number of concurrent tasks during the job execution.

$$
A_{p}=\sum_{n=1}^{N}\left(n P_{p}(n)\right)
$$

where $N$ is the total number of client tasks in the client system.

- Client task $t$ active time $e(t)$. ( $t$ is a node in precodence graph).

$$
e(t)=T_{c} \sum_{1 \in E} P_{s}(i)
$$

where $E$ is the set of states in which client task $t$ is executing.

- Client rask $t$ utilization $U(t)$

$U(t)$ is the utilization of client task $t$ as the percentage of time client task $t$ is "busy" (computation or waiting for RV replies) with respect to the completion time of the whole precedence graph. 


$$
U(t)=e(t) / T_{c}
$$

\subsubsection{Performance Measures for Multiple Clients RNMTC System}

In multiple client system, we are more interested in the performance of individual client.

- Client Throughput $X(g)$ in class $g$

Due to the fact that all clients in a class are statistically identical, we only need to compute the throughput for a client, let this be client 1 . Select a reference client task $t$ in client 1 in the precedence graph. The node $t$ is visited only once in one instance. $\mu(i, t)$ is the transition rate $e f$ termination of node $t$ from MC state $s$.

$$
X_{\gamma}(g)=\sum_{i \in Y}\left(P_{s}(i) \mu(i, t)\right)
$$

Figure 4.5 shows 2 classes of clients with one client each. They have the same structure of precedence graph but have different computation time for client tasks so that they belong to different classes.

Class 1

Client 1

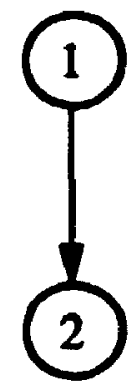

Class 2

Client 1

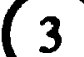

4

Figure 4.5. Multiple client system 
Figure 4.6 shows the MC for the two classes system shown in Figure 4.5. In order to compute the throughput $X_{\mathbb{N}}(2)$ for class 2 , node 4 is selected as a reference node of the client. Node 4 is active in state $S_{3}$ and state $S_{3} . \mu\left(S_{3}, 4\right)$ is the transition rate corresponding to the termination of node 4 in state $S_{3}$ while $\mu\left(S_{4}, 4\right)$ is the transition rate corresponding to the termination of node 4 in state $S_{4} . P_{s}\left(S_{3}\right)$ and $P_{s}\left(S_{4}\right)$ are state $S_{3}$ and $S_{3}$ probabilities, respectively. Therefore,

$$
x_{\mathbb{N}}(2)=\operatorname{Ps}\left(S_{3}\right) \mu\left(S_{3}, 4\right)+\operatorname{Ps}\left(S_{4}\right) \mu\left(S_{4}, 4\right)
$$

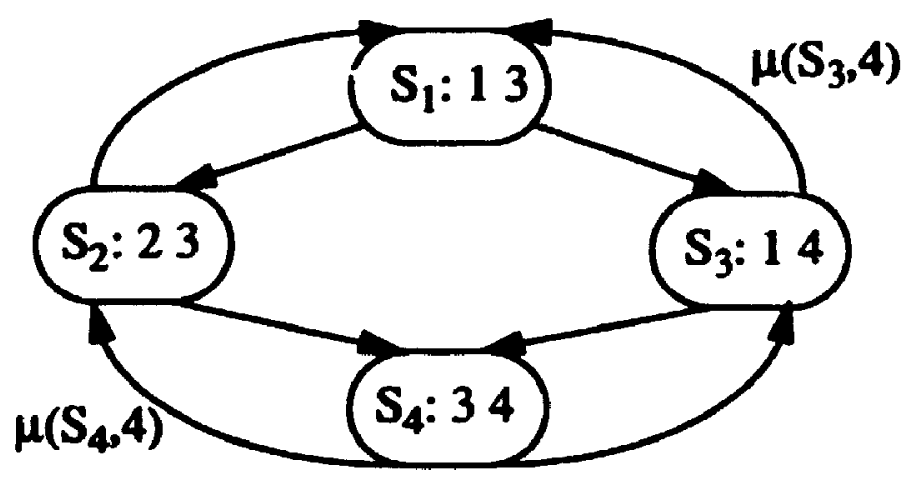

Figure 4.6. MC states

- Class throughput $\ddot{d} d g)$ in class $g$

If there are $C_{R}(g)$ clients in class $g$, class throughput is expressed as follows:

$$
X_{c}(g)=C_{l}(g) X_{h}(g)
$$

- Jient cycle time $T_{c l}(g)$ in class $g$

$$
T_{c}(g)=1 / X_{i}(g)
$$

- Parallelism distributions $P_{p}(\boldsymbol{n})$

This is the same definition and computation as for the single client system. 


$$
P_{p}(n)=\sum_{i \in R} p_{s}(i)
$$

- Average parallelism $A_{p}$

Average parallelism $A_{p}$ is the average number of concurrent tasks during the job execution.

$$
A_{p}=\sum_{n=1}^{Z}\left(n P_{p}(n)\right)
$$

where $Z$ is the total number of client tasks in the multiple client RNMTC system.

$$
Z=\sum_{i=1}^{C_{4}} c(i) N(i)
$$




\section{Chapter 5}

\section{Bounds}

\subsection{Motivation}

Analytic ta:hniques are used widely in the performance evaluation of concurrent systems. Test cases described in chapter 6 will prove that the algorithm presented in Chapter 4 produces a reasonable degree of accuracy in evaluating system performance. The algorithm, however, is based on assumptions about distribution of execution times and service times which may not always be satisfied on certain mnvironments. Performance bounds which are based on weaker assumptions about system bzhavior can be used in such a situation.

The bounds presented in this chapter are applicable to systems with fixed computation and service time and general distributions of computation times and FIFO service at servers. These weaker conditions make the bounds robust and applicable to a wide range of systems characterized by rendezvous style communications. For systems in which the distribution of computation times are unknown, the exact analytic analysis may be intractable anci bounds may be the only performance result that can be computed for such systems.

Upper bounds and lower bounds on task throughputs for a SRVN model are presented in [MWNP91]. The authors extract a set of bounds for several kinds of system: system with single reference task or multiple reference tasks, systems characterized by processor contention etc. But [MWNP91] only discusses the bounds based on the assumption that each server has only a single entry and the tasks have no precedence relationship. This research expands the bounds analysis to RNMTC model characterized by multiple entry servers and single multi-threaded client. The 
servers are restricted to be pure servers; that is, the server submodel has a single level without nested requests between servers. Somputation of the upper and lower bounds on client completion times for certain classes of the RNMTC are presented in this chapter. The following specific types of RNMTC system are studied in this thesis:

- Deterministic system (section 5.3)

- Systems with fixed computation times and service times (section 5.3.1)

- Systems with general computation and service time distribution (section 5.3.2)

- Systems with probabilistic branching (section 5.4)

- Systems with loop construct (section 5.5)

\subsection{Parameters}

As stated in section 2.2, a node precedence graph is used to model a client system. A client cycle starts with the start of execution of initialization node(s) (or initialization client task) and ends with the completion of execution of termination node(s) (or termination client task). In order to compute the performance bounds for a client, some notations must be defined first. Some of these are already defined in section 4.4 in the content of the approximate analysis. However for the sake of clarity, these are listed here again.

$N=$ total number of client tasks in the client

$T_{c}=$ client $\iota$. He time

$\Rightarrow$ the difference between the time at which the initialization client tasks stan execution and the time at which all termination client tasks complete

$c(t)=$ average computation time for client task $t$

$e(t)=$ average execution time for client task $t$

$e(t)$ includes $c(t)$ and also service time and queueing delay 
$v(t, j, u)=$ average number of visits (or visit ratio) made by client task $t$ to entry $u$ of server $j$

$v(t, j)=$ average number of visits (or visit ratio) made by client task $t$ to server $j$

$$
v(t, j)=\sum_{u=1}^{U} v(t, j, u)
$$

where $U$ is total number of entries in server $j$. If only a single entry exist, $v(t, j, u)$ can be expressed as $v(t, j)$.

$e_{s}(t)=$ time at which client task $t$ starts to execute

$e(t)=$ time at which client task $t$ completes execution

$t<\bullet l \Rightarrow$ client task $t$ is a direct predecessor of client task $l$

$t<0 * l \Rightarrow$ client task $t$ is a direct or indirect predecessor of client task $l$ (which means that $t$ $<\bullet l$ or $t<\bullet z$ and $z<0 * t)$

$t \bullet>l \Rightarrow$ client task $t$ is a direct successor of client task $l$

$t \bullet>* l \Rightarrow$ client task $t$ is a direct or indirect successor of client task $l$ (which means that $t \bullet>$ $l$ or $t \bullet>z$ and $\left.z \bullet \bullet^{*} l\right)$

$T(t)=$ set of all direct predecessors of client task $t$

$P^{*}(t)=$ set of all predecessors (direct or indirect) of client task $t$

$S(t)=$ set of all direct successors of client task $t$

$S^{*}(t)=$ set of all successors (direct or indirect) of client task $t$

$C(t)=$ concurrency set for client task $t$ (set of all client tasks that can execute concurrently with client task $t$ )

$K=$ tutal number of servers in the system

$s(i, e, p)=$ average service time for entry $e$ in phase $p(p=1$ or $p=2)$ at server $i$ per visit 
$s(i, e)=$ average service time for entry $e$ at server $i$ (both phases included)

$$
s(i, e)=s(i, e, 1)+s(i, e, 2)
$$

$P\left(t_{1}, t_{2}, k\right)=$ probability that client task $t_{1}$ is delayed by client task $t_{2}$ at server $k$.

$$
\text { where } t_{2} \in C\left(t_{1}\right)
$$

The bounds analysis is based on the following lemma.

Lemma 1. $P\left(t_{1}, t_{2}, k\right) \leq \min \left(1, \frac{v\left(t_{2}, k\right)}{v\left(t_{1}, k\right)}\right)$

Proof: A technique similar to the one presented in [MWNP92] is used for proving the lemma. Consider client task $t_{1}$ and client task $t_{2}$ make requests to server $k$ at which FIFO discipline is exploited. The mean number of visits made during one job cycle hy $t_{1}$ and $t_{2}$ are given by $v\left(t_{1}, k\right)$ and $v\left(t_{2}, k\right)$ respectively.

- Case $1: \frac{v\left(t_{2}, k\right)}{v\left(t_{1}, k\right)}>1$

$P\left(t_{1}, t_{2}, k\right)$ is a probability that cannot exceed 1 .

- Case $2: \frac{v\left(t_{2}, k\right)}{v\left(t_{1}, k\right)} \leq 1$

By contradiction, suppose that $P\left(t_{1}, t_{2}, k\right)>v\left(t_{2}, k\right) / v\left(t_{1}, k\right)$. Let $q\left(t_{1}, t_{2}\right)$ be the mean number of visits during one job cycle of $t_{1}$ in which $t_{1}$ found $t_{2}$ already enqueved or in service at server $\boldsymbol{k}$.

Since the mean number of visits made by $t_{2}$ during one job cycle is $v\left(t_{2}, k\right)$, we have:

$$
q\left(t_{1}, t_{2}\right) \leq v\left(t_{2}, k\right)
$$

Now $P\left(t_{1}, t_{2}, k\right)=q\left(t_{1}, t_{2}\right) / v\left(t_{1}, k\right)$

Thus $P\left(t_{1}, t_{2}, k\right)>v\left(t_{2}, k\right) / v\left(t_{1}, k\right)$ implies 


$$
q\left(t_{1}, t_{2}\right)>v\left(t_{2}, k\right)
$$

This is in contradiction with 5-1.

Based on the analysis of case 1 and case 2, we get

$$
P\left(t_{1}, t_{2}, k\right) \leq \min \left(1, \frac{v\left(t_{2}, k\right)}{v\left(t_{1}, k\right)}\right)
$$

\subsection{Bounds on the Performance of Deterministic System}

A deterministic system is characterized by an AND parallelism: after the compietion of a task, all of its successors star: to execute. Each client task or server is assumed to be executing on its own processor so that there is no contention for hardware. But contention for software servers may exist.

First, we discuss the lower bound and upper bound on client task execution times in the system.

The lower bound is based on the optimistic situation in which each client task is served without any queueing delay at a server. Therefore, the lower bound on execution time for client task $t, e^{-}(t)$, includes the task's own computation time $c(t)$ and the total first phase service time at each server visited by $t$. Thus,

$$
e(t) \geq e^{e}(t)=c(t)+\sum_{k=1}^{K} \sum_{u=1}^{U} v(t, k, u) s(k, u, 1)
$$

The upper bound on the execution time for client task $t, e^{+}(t)$, not only includes all the terms included in 5-2, but also the queueing delay experienced by client task $t$ at each server. In the worst case, whenever client bask $t$ arrives at server $k$, the server is executing its longest post RV phase corresponding to a previous request; this term is expressed by $\Sigma v(t, k) \max (s(k, u, 2))$. Each task $y$ in the concurrency sei $C(t)$ can introduce a queueing delay for $t$ at any server. The upper bound on this queueing delay experienced by $t$ per visit of $k$ is given by $P^{+}(t, y, k) s(k, \mu)$, where $P^{+}(t, y, k)$ is the upper bound on the probability that $y$ is enqueued at $k$ when $t$ arrives. If $y$ visits multiple entries of $k$ then $u$ is that entry for which the average service time (including both phases) 
is the highest. Thus,

$$
\begin{aligned}
e^{+}(t)=c(t) & +\sum_{k=1}^{K} \sum_{u=1}^{U} v(t, k, u) s(k, u, 1) \\
& +\sum_{k=1}^{K} v(t, k) \max _{u=1}^{U}(s(k, u, 2))+\sum_{k=1}^{K} v(t, k) \sum_{y \in C(t)} \sum_{u=1}^{U} P^{+}(t, y, k) s(k, u)
\end{aligned}
$$

Where $P(t, y, k)$ is the probability of client task $t$ being delayed by $y$ at server $k$. As shown in lemma 1:

$$
P(t, y, k) \leq P^{+}(t, y, k)=\min \left(1, \frac{v(y, k)}{v(t, k)}\right) .
$$

Therefore,

$$
\begin{aligned}
e(t) \leq e^{+}(t)=c(t) & +\sum_{k=1}^{K} \sum_{k=1}^{U} v(t, k, u) s(k, u, 1) \\
& \left.+\sum_{k=1}^{K} v(t, k) \underset{u=1}{U} \max _{u=1}(k, k, 2)\right) \\
& +\sum_{k=1}^{K} v(t, k) \sum_{u=1}^{U} \sum_{y \in C(t))} \min \left(1, \frac{v(y, k)}{v(t, k)}\right) s(k, u)
\end{aligned}
$$

The bound on client completion time is based on the execution times of its client tasks and is presented next.

\subsubsection{Bounds on Systems with Fixed Computation Times and Service Times}

Inequalities are derived in previous subsection for computin? bounds on the execution time of individual client task execution times. This section discusses the bounds on client completion time for systems in which client task computation times and service times of servers are fixed.

Since a client task $t$ can start execution only when all its predecessors have completed. We have: 


$$
e^{\prime} y=\max _{t \in F(t)}\{e(t)\}
$$

The time at which $t$ completes is giver: hy

$$
e_{f}(t)=e_{s}(t)+e(t)
$$

Since a job completes when all its tasks have completed, we obtain the following expression for the job completion time:

$$
T_{c}=\max _{t=1}^{N}(e(t))
$$

Let $e_{s}^{+}(t)$ be the latest starting time for client task $t, e_{f}^{+}(t)$ be the latest completion time for $t$. Client task $t$ can start only when all its predecessors have completed execution, thus

$$
e_{s}^{+}(t)=\max _{\in \in T(t)}\left\{e_{f}^{+}(t)\right\}
$$

The upper bound on completion time of $t$ is equal to the sum of its latest starting time and the upper bound on its execution time:

$$
e_{f}^{+}(t)=e_{s}^{+}(t)+e^{+}(t)
$$

From 5-3 and 5-5, we get

$$
\begin{aligned}
& e_{f}^{+}(t)=e_{s}^{+}(t)+c(t)+\sum_{k=1}^{K} \sum_{k=1}^{U} v(t, k, u) s(k, u, 1) \\
& +\sum_{k=1}^{x} v(t, k) \underset{\max (s(k, u, 2))}{U} \\
& +\sum_{k=1}^{K} v(t, k) \sum_{u=1}^{U} \sum_{C(t))} \min \left(1, \frac{v(y, k)}{v(t, k)}\right) s(k, u)
\end{aligned}
$$

Since the client completes execution when its last client task completes, the upper bound on 
$T_{c}$ is given by the following expression:

$$
T_{c}^{+}=\max _{t=1}^{N}\left\{e_{f}^{+}(t)\right\}
$$

The derivation of a lower bound on $T_{c}$ is presented next:

Let $e_{f}^{-}(t)$ be the earliest completion time for client task $t, e_{s}^{-}(t)$ be the earliest starting time for $t$. Then,

$$
e_{s}^{-}(t)=\max _{t \in A(t)}\left\{e_{f}^{-j}(t)\right\}
$$

Then, the lower bound on execution time of $t$ is given by

$$
e_{f}^{-}(t)=e_{s}^{-}(t)+e^{-}(t)
$$

Where $e^{-}(t)$ is the lower bound on the execution time of $t$.

From 5-2 and 5-7 we get

$$
e_{f}^{-}(t)=e_{s}^{-}(t)+c(t)+\sum_{k=1}^{K} \sum_{u=1}^{U} v(t, k, u) s(k, u, 1)
$$

The lower bound on client cycle time is then computed as:

$$
T_{c}^{-}=\max _{t=1}^{N}\left\{e_{j}(t)\right\}
$$

Computation of bounds for system with general computation and service time distributions is presented next. 


\subsubsection{Bounds on Systems with General Computation and Service Time Distribution}

The bounds derived from fixed time systems are not valid for systems in which the computation time of client tasks and service time of servers are not fixed. A class of "fork-join" systems in which the client task execution times and server service times (in each phase) are characterized by IFR (Increasing Failure Rate) distribution is considered here.

Consider a client with $n+2$ client tasks from $t_{0}$ to $t_{n+1}$. The precedience graph for the client is shown in Figure 5.1. Client tasks $t_{0}$ and $t_{n+1}$ have no requests 10 any servers while the client tasks from $t_{1}$ to $t_{n}$ may have requests to servers. Each client task executes on its own processor.

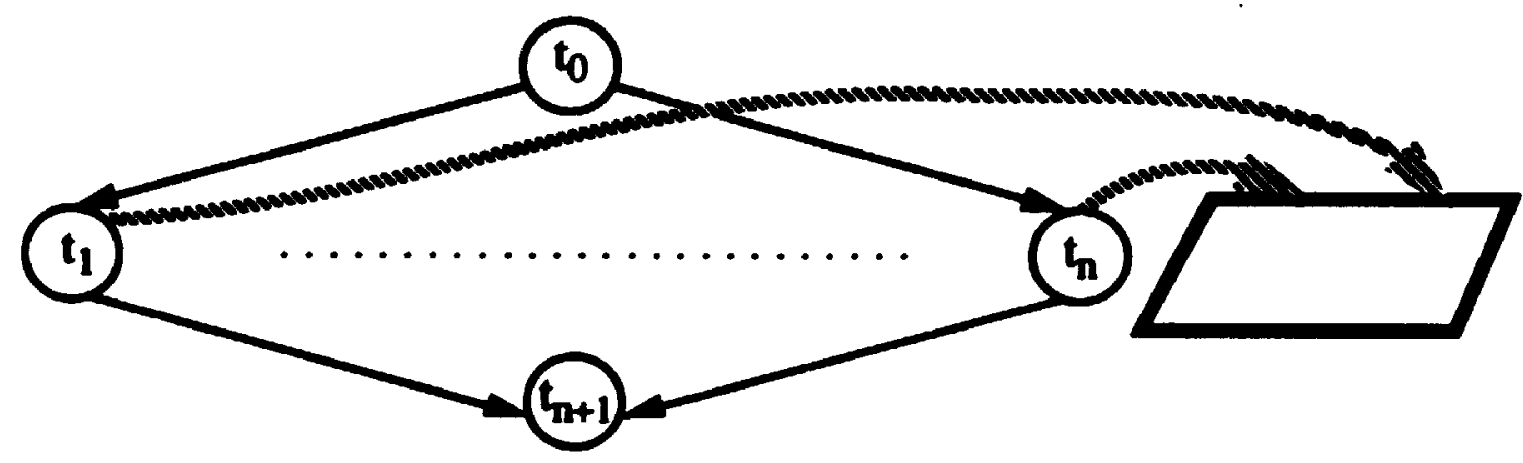

Client submodel

Server submodel

Figure 5.1. Concurrent task in unbounded system

The expected client cycle time is given by

$$
T_{c}=e\left(t_{0}\right)+E(\lambda)+e\left(t_{\mathrm{n}+1}\right)
$$

Where $E(\lambda)$ is the expected time interval measured from the termination of client task to to 
the beginning of client task $t_{n+1}$.

The client cycle time is thus bounded as described below.

$$
\begin{aligned}
& T_{c} \leq T_{c}^{+}=e^{+}\left(t_{0}\right)+e^{+}\left(t_{n+1}\right)+E^{+}(\lambda) \\
& T_{c} \geq T_{c}^{-}=e^{-}\left(t_{0}\right)+e^{-}\left(t_{n+1}\right)+E(\lambda)
\end{aligned}
$$

In order to obtain the upper and lower bounds on $T_{c}$, we need to compute $E^{+}(\lambda)$ and $E(\lambda)$.

Clearly,

$$
E(\lambda)=E\left[\max \left(e^{-}\left(t_{1}\right), e^{-}\left(t_{2}\right), \ldots, e^{-(}\left(t_{n}\right)\right)\right]
$$

Where $e^{-}\left(t_{\mathrm{i}}\right)(i=1, \ldots, n)$ is a random variable for the execution time of client task $t_{\mathrm{i}}$.

Noting that $\lambda$ is the random variable for the time interval measured from the termination of $t_{0}$ to the beginning of $t_{n+1}$, we get

$$
\lambda=\max \left(e^{-}\left(t_{1}\right), e^{-}\left(t_{2}\right), \ldots e^{-}\left(t_{n}\right)\right)
$$

\section{Clearly,}

$$
\lambda \leq e^{-}\left(t_{1}\right)+e^{-}\left(t_{2}\right)+\ldots+e^{-}\left(t_{n}\right)
$$

By using 5-11 and noting that $e^{+}\left(t_{i}\right)$ is an upper bound on $e\left(t_{i}\right)$, we get:

$$
E(\lambda) \leq E^{+}(\lambda)=e^{+}\left(t_{1}\right)+e^{+}\left(t_{2}\right)+\ldots+e^{+}\left(t_{n}\right)=\sum_{m=1}^{n} e^{+}\left(t_{m}\right)
$$

Therefore, from inequalities 5-9 and 5-13, we get 


$$
T_{c}^{+}=e^{+}\left(t_{0}\right)+e^{+}\left(t_{n+1}\right)+\sum_{m=1}^{n} e^{+}\left(t_{m}\right)
$$

Because client task $t_{0}$ and $t_{n+1}$ have no requests to servers, their execution times only include their computation times. That is,

$$
\begin{aligned}
& e^{+}\left(t_{0}\right)=c\left(t_{0}\right) \text { and } \\
& e^{+}\left(t_{n+1}\right)=c\left(t_{n+1}\right)
\end{aligned}
$$

Therefore, from 5-14, the upper bound on $T_{c}$ can be described by

$$
T_{c}^{+}=c\left(t_{0}\right)+c\left(t_{n+1}\right)+\sum_{m=1}^{n} e^{+}\left(t_{m}\right)
$$

Note that $e^{+}\left(t_{i}\right)(i=1, \ldots, n)$ can be found by using 5-3.

Following the derivation of 5-3, one observes that

$$
\begin{aligned}
e(t) \leq e^{+}(t)=c(t) & +\sum_{k=1}^{K} \sum_{k=1}^{U} v(t, k, u) s(k, u, 1) \\
& +\sum_{k=1}^{K} v(t, k) R_{k}^{+}+\sum_{k=1}^{K} Q^{+}(t, k)
\end{aligned}
$$

Where $\sum_{k=1}^{k} Q^{+}(t, k)$ is upper bound of the queueing delay (cover all visits) experienced by client task $t$ at server $k$ with other concurrent client tasks.

$$
\sum_{k=1}^{K} Q^{+}(t, k)=v(t, k) \sum_{u=1}^{U} \sum_{y \in c(t))} P^{+}(t, y, k) S(k, u)
$$

$R_{k}{ }^{+}$is the upper bound on the expected remaining time for the completion of the post RV phase of service due to a previous server request. 
Since the second phase service times at any entry $u$ of $k$ is IFR distributed, the remaining service time when $t$ arrives is upper bounded by $s(k, u, 2)[\mathrm{Kle}]$. Thus,

$$
R_{k}^{+}=\underset{\substack{u=1 \\ u=1}}{U}(s(k, u, 2))
$$

Now by using Lemma 1 , equation 5-16, 5-17 and 5-18, $T_{c}^{+}$can be derived as in 5-15.

The lower bound $T_{c}^{-}$can be derived in the following manner.

From equation 5-12, we can get

$$
\lambda=\max \left(e^{-}\left(t_{1}\right), e^{-}\left(t_{2}\right), \ldots, e^{-}\left(t_{n}\right)\right)
$$

Thus $\lambda \geq e^{\tilde{e}}\left(t_{i}\right)$ for $i=1, \ldots, n$ and $E(\lambda) \geq E\left[e^{-}\left(t_{i}\right)\right]$ for $i=1, . ., n$.

Noting that $e^{-}\left(t_{i}\right)$ is a lower bound on $E\left[e^{-}\left(t_{i}\right)\right]$, we get:

$$
E(\lambda) \geq E(\lambda)=\max \left(e^{-}\left(t_{1}\right), e^{-}\left(t_{2}\right), \ldots, e^{-}\left(t_{n}\right)\right)
$$

Now by using $5-10$, we get:

$$
T_{c}^{\cdot}=e^{-}\left(t_{0}\right)+e^{-}\left(t_{n+1}\right)+\max \left(e^{-}\left(t_{1}\right), e^{-}\left(t_{2}\right), \ldots, e^{-}\left(t_{n}\right)\right)
$$

Since $e^{-}\left(t_{0}\right)=c\left(t_{0}\right)$ and $e^{-}\left(t_{n+1}\right)=c\left(t_{n+1}\right)$, we get:

$$
T_{c}^{-}=c\left(t_{0}\right)+c\left(t_{n+1}\right)+\max \left(e^{-(}\left(t_{1}\right), e^{-(}\left(t_{2}\right), \ldots, e^{-}\left(t_{n}\right)\right)
$$

Note that $e^{-}\left(t_{i}\right)(i=1, \ldots, n)$ can be found by using $5-2$.

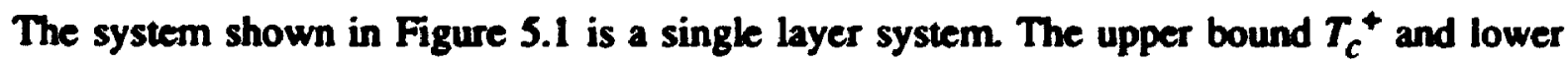
bound $T_{c}^{-}$derived in 5-15 and 5-20 for such a system can be expanded from single layer to multi- 
ple layers shown in Figure 5.2.

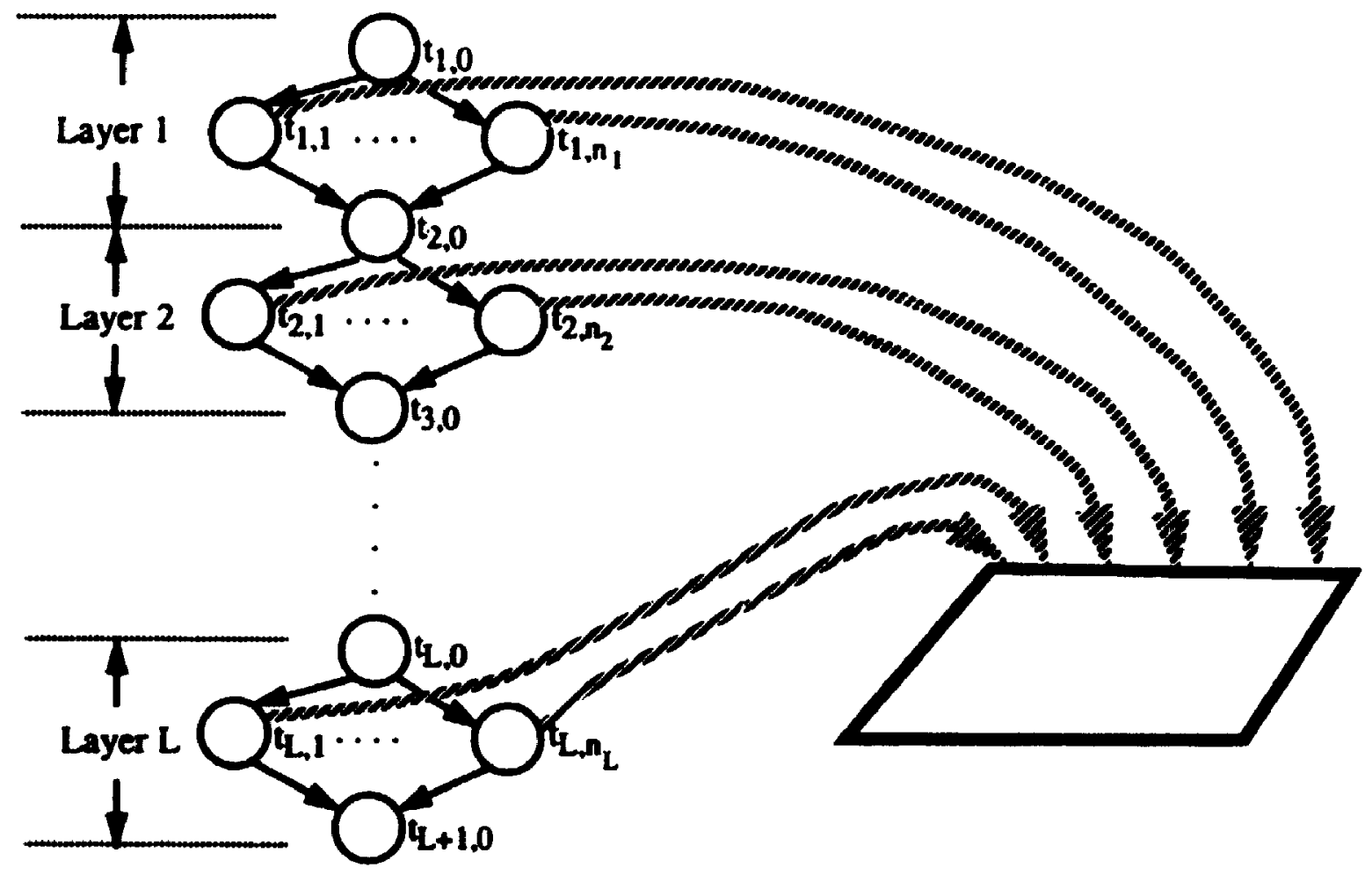

Client submodel

Server submodel

Figure 5.2. Multiple layer system

There are $L$ layers in the system. There are $n_{l}$ concurrent client tasks in layer $l_{.} t_{1 j}$ in Figure 5.2 is client task $i$ in layer $l$. In each layer, the concurrent client tasks join at the same client task (e.g. $\left.t_{2,0}, \ldots, t_{L, 0}\right)$ and the concurrent client tasks in next layer fork from these client tasks. Client tasks $t_{1,0} \ldots . ., t_{L, 0}$ have no service requests to servers whereas other client tasks may make calls to servers.

Let $T_{c l}$ be the completion time for layer $l . T_{c l}$ is the time between the beginning of client task $t_{1,0}$ to the termination of client task $t_{l+1,0} T_{c l}{ }^{+}$and $T_{c l}$ are upper bound and lower bound on $T_{c l}$ respectively and can be found by using 5-15 and 5-20. 


$$
\begin{aligned}
& T_{c l}{ }^{+}=c\left(t_{l, 0}\right)+c\left(t_{l+1,0}\right)+\sum_{m=1}^{n_{l}} e^{+}\left(t_{l, m}\right) \\
& T_{c l}=c\left(t_{l, 0}\right)+c\left(t_{l+1,0}\right)+\max \left(e^{-}\left(t_{l, 1}\right), e^{\left.-\left(t_{l, 2}\right) \ldots . . e^{-}\left(t_{l, n_{l}}\right)\right)}\right.
\end{aligned}
$$

where $c\left(t_{t+1,0}\right)$ in 5-21 and 5-22 is equivalent to $c\left(t_{n+1}\right)$ in 5-15 and 5-26.

Let $T_{c}$ be the completion time for the multiple layer system. Let $T_{c}{ }^{+}$and $T_{c}{ }^{-}$are upper bound and lower bound on $T_{c}$. Noting that the execution times of client tasks from $t_{2,0} \ldots, t_{L}, 0$ appear in the completion time for the layers, we get:

$$
T_{c}^{+}=\sum_{l=1}^{L} T_{c l}^{+}-T_{0}
$$

Where

$$
T_{0}=\sum_{i=2}^{L} c\left(t_{l, 0}\right)
$$

From 5-21 and 5-23 we get

$$
T_{c}^{+}=\sum_{l=1}^{L}\left(c\left(t_{l, 0}\right)+c\left(t_{l+1,0}\right)+\sum_{m=1}^{n_{l}} e^{+}\left(t_{l, m}\right)\right)-\sum_{l=2}^{L} c\left(t_{l, 0}\right)
$$

From 5-20, we can derive the lower bound on $T_{c}$.

$$
T_{c}=\sum_{l=1}^{L} T_{c i}-T_{0}
$$

From 5-22 and 5-23 we can get

$$
\begin{aligned}
T_{c}^{-}= & \sum_{i=1}^{L}\left(c\left(t_{l, 0}\right)+c\left(t_{l+1,0}\right)+\max \left(e^{-}\left(t_{l, 1}\right), e^{-}\left(t_{l, 2}\right), \ldots, e^{-}\left(t_{l, n_{1}}\right)\right)\right) \\
& -\sum_{i=2}^{L} c\left(t_{l, 0}\right)
\end{aligned}
$$




\subsection{Bounds on the Performance of Systems with Probabilistic Branching}

In a precedence graph with probabilistic branching, the client tasks may have OR relationships with one another. All of the successors of $z$ client task may not start execution after the completion of the client task. There is a branching probability $b_{j}$ associated with the execution of each successor $j$ of a source task $i$.

Consider a simple system shown in Figure 5.3 first.

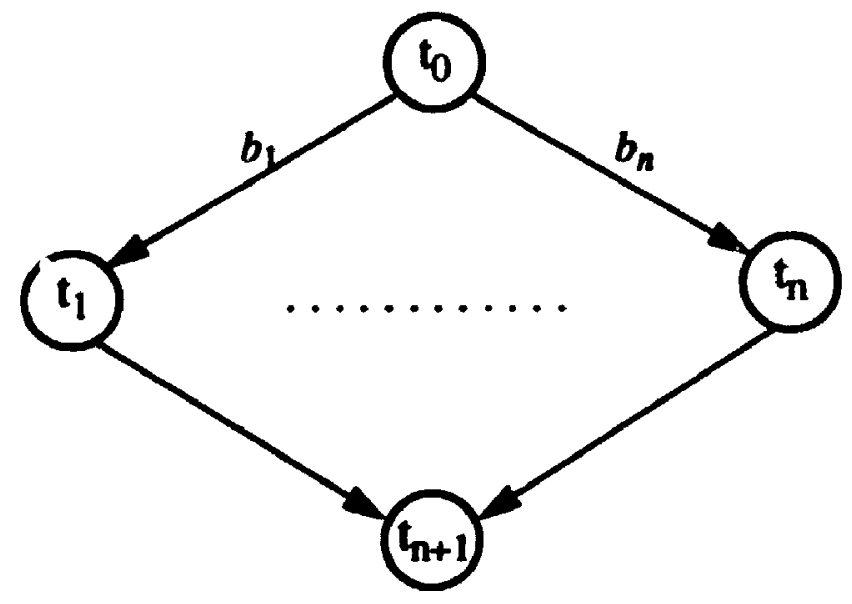

Figure 5.3. Simple probabilistic branching

In this system, assume there is no server submodel and after the termination of client task $t_{0}$. only one of its successers $t_{i}(i=1, \ldots, n)$ starts execution. There are multiple execution paths in the system, such as $t_{\sigma}>t_{1}>t_{n+1}, t_{\sigma^{-}}>t_{n}->t_{n+1}$. ?ath $t_{\sigma^{-}}>t_{i}>t_{n+1}$ is called path $i$. Let $T_{c i}$ be the completion time for the system when path $i$ is taken. The expected completion time $T_{c}$ for this system is then given by

$$
T_{c}=b_{1} T_{c l} \div b_{2} T_{c 2}+\ldots+b_{n} T_{c n}=\sum_{i=1}^{n} b_{i} T_{c i}
$$

Therefore 


$$
T_{c}^{+}=\sum_{i=1}^{n} b_{i} T_{c i}^{+}
$$

and

$$
T_{c}^{-}=\sum_{i=1}^{n} b_{i} T_{c i}
$$

Now consider a more complicated probabilistic branching system in Figure 5.4 .

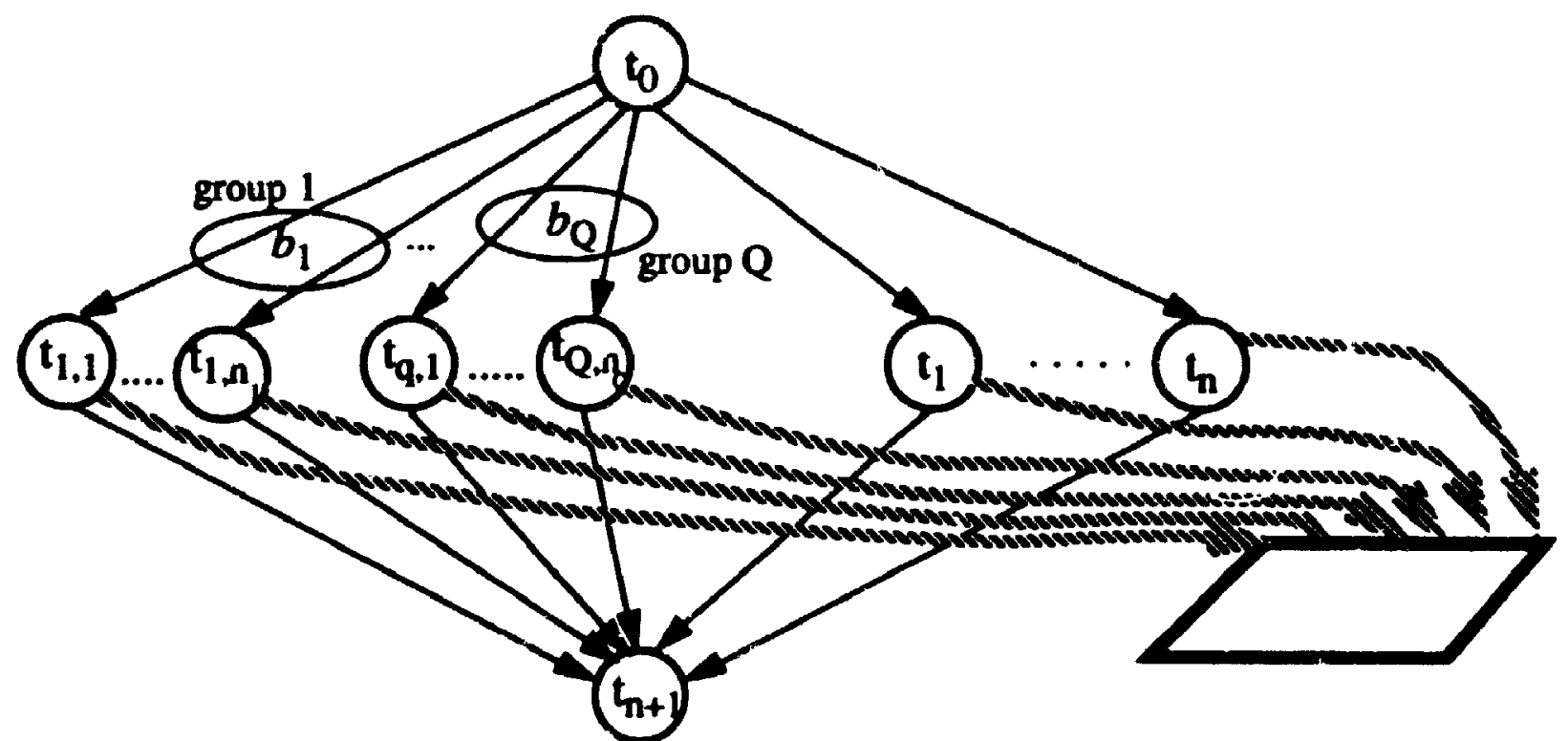

Client submodel

Server submodel

Figure 5.4. Probabilistic branching

The initializasion client task is $t_{0}$ and the termination client task is $t_{n+1}$. Neither of them makes any request to servers. Other client tasks mp.v make requests to servers during their execution. There are two classes of successor client tasks: AND class and OR class. For an AND class (xa) all the client tasks will start execution upon the completion of their predecessors. The client tasks from $t_{l}$ to $t_{n}$ in Figure 5.4 belong to the AND class client tasks. The client tasks in the OR class $(X O)$ may or may not start execution after their predecessors finish. $t_{I, I}$ and $t_{Q, n_{Q}}$ are two examples of OR class client tasks. The OR class client tasks are grouped with an ellipse. There are 
$Q$ groups in the system. Each group has a probability associated with it $\left(b_{1}, \ldots, b_{Q}\right)$ and group $q$ contains $n_{q}$ multiple client tasks. For example, the branching probability for group 1 is $b_{1}$ and group 1 has $n_{1}$ client tasks from $t_{1,1}$ : $t_{1, n_{1}}$. After the termination of $t_{0}$. group $q$ is chosen to execute with a probability $b_{q}$ and all the client tasks in group $q$ are activated. Note that

$$
\sum_{q=1}^{Q} b_{q}=1
$$

The concurrency set of client task $t_{q j}$ includes client tasks in both classes: all of the client tasks in $x q$ and the OR class client tasks $t_{q t}$ which arc in group $q$.

Let $T_{c q}$ be the completion time for system shown in Figure 5.4 when group $q$ is executed. Also let $T_{c q}{ }^{+}$and $T_{c q}{ }^{-} b$ - upper 'nd lower bounds on $T_{c q}$. Using 5-15, we get

$$
T_{c q}{ }^{+}=c\left(t_{0}\right)+c\left(t_{n+1}\right)+\sum_{m=1}^{n} e^{+}\left(t_{m}\right)+\sum_{i=1}^{n_{1}} e^{+}\left(t_{q, i}\right)
$$

Using 5-20 yields

$$
\begin{aligned}
T_{c q^{-}=}= & c\left(t_{0}\right)+c\left(t_{n+1}\right) \\
& +\max \left(e^{-}\left(t_{1}\right), e^{-}\left(t_{2}\right), \ldots, e^{-}\left(t_{n}\right), e^{-}\left(t_{q, 1}\right), \ldots, e^{-(}\left(t_{q, n_{q}}\right)\right)
\end{aligned}
$$

Let $T_{c}$ be the completion time for the system shown in Figure 5.4. From 5-27 and 5-29, we get

$$
T_{c}^{+}=\sum_{q=1}^{Q} b_{q}\left(c\left(t_{0}\right)+c\left(t_{n+1}\right)+\sum_{m=1}^{n} e^{+}\left(t_{m}\right)+\sum_{i=1}^{n_{q}} e^{+}\left(t_{q, i}\right)\right)
$$

Noting that $\sum_{q=1}^{Q} b_{q}=1,5-31$ yields 


$$
T_{c}^{+}=c\left(t_{0}\right)+c\left(t_{n+1}\right)+\sum_{m=1}^{n} e^{+}\left(t_{m}\right)+\sum_{q=1}^{Q} b_{q} \sum_{i=1}^{n_{q}} e^{+}\left(t_{q, i}\right)
$$

From 5-28 and, we get

$$
\begin{aligned}
T_{c} \cdot= & \sum_{q=1}^{Q} b_{q}\left(c\left(t_{0}\right)+c\left(t_{n+1}\right)\right. \\
& \left.+\max \left(e^{-}\left(t_{1}\right), e^{-}\left(t_{2}\right), \ldots, e^{-}\left(t_{n}\right), e^{-(}\left(t_{q, 1}\right) \ldots, e^{-}\left(t_{q, n_{q}}\right)\right)\right)
\end{aligned}
$$

Again because $\sum_{q=1}^{Q} b_{q}=1,5-33$ yields

$$
\begin{aligned}
T_{c}^{-}=c\left(t_{0}\right) & +c\left(t_{n+1}\right) \\
& +\sum_{q=1}^{\ell} b_{q} \max \left(e^{-}\left(t_{1}\right), e^{-(}\left(t_{2}\right), \ldots, e^{-}\left(t_{n}\right), e^{-}\left(t_{q, 1}\right), \ldots, e^{-}\left(t_{q, n_{q}}\right)\right)
\end{aligned}
$$

The bounds derived in 5-32 and 5-34 for the system shown in Figure 5.4 can be expanded to systems with the multiple layers. Figure 5.5 is a multiple layer system and there may be some probabilistic branching in each layer. There are $L$ layers in Figure 5.5. The "join" task in one layer serves as the "fork" task in the next layer. The fork/join tasks do not make service requests to servers that may be visiterl by other client tasks. The number of AND class tasks in layer $l$ is $\eta_{l}$. A client task $t_{l, j}$ is jth cliers task in the AND class of layer $l$. In Figure 5.5 group $(l, q)$ is the qth group in layer $l$. There are $Q_{l}$ groups of OR tasks in layer $l$ and the $q$ th group in layer $l$ contains $n_{l q}$ client tasks. $b_{l q}$ is the branching probability for group $q$ in layer $l$. An OR class client task $t_{l, q, t}$ is the $k$ th client task in group $q$ of layer $l$.

Let $T_{c l}$ be the conipletion time for layer $l$ and $T_{c}$ be the completion time for the multiple layer system. From 5-32 and 5-34, we can get the upper and lower bounds for layer $l(l=1, \ldots L)$. $c\left(t_{l+1,0}\right)$ in 5-35 and 5-36 is equivalent "o $c\left(t_{n+1}\right)$ in 5-32 and 5-34. Thus $T_{c l}{ }^{+}$is given in the following manner: 


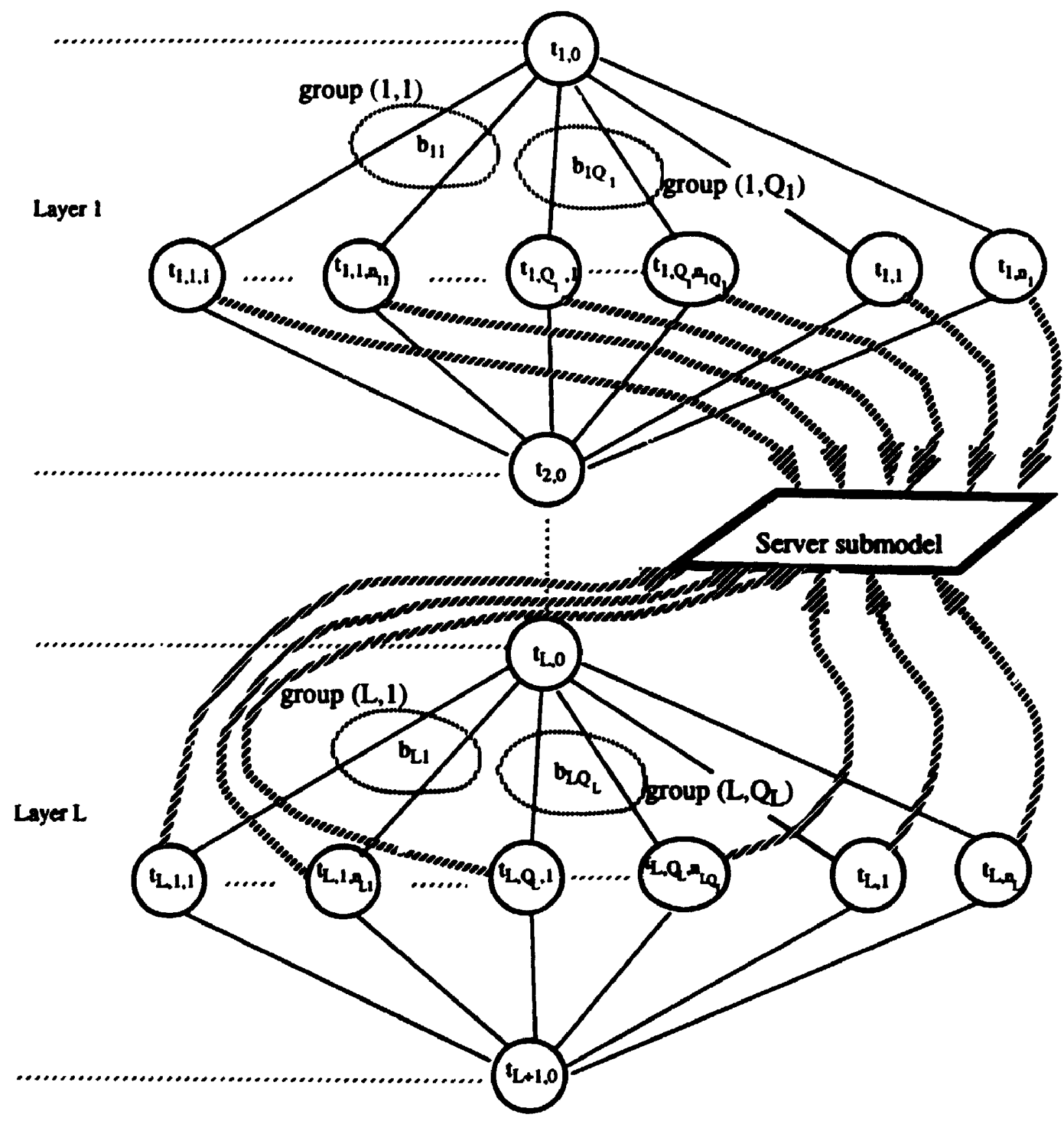

Figure 5.5. Multiple layer probabilistic branching system 


$$
T_{c l}{ }^{+}=c\left(t_{l, 0}\right)+c\left(t_{l+1,0}\right)+\sum_{m=1}^{n_{1}} e^{+}\left(t_{l, m}\right)+\sum_{q=1}^{Q_{1}} b_{l q} \sum_{i=1}^{n_{l,}} e^{+}\left(t_{l, q, i}\right)
$$

and $T_{c l}$ is given by:

$$
\begin{aligned}
T_{c l}= & c\left(t_{l, 0}\right)+c\left(t_{l+1}, j\right. \\
& +\sum_{q=1}^{Q_{l}} b_{l q} \max \left(e^{-(}\left(t_{l, 1}\right), e^{-}\left(t_{l, 2}\right), \ldots, e^{-(}\left(t_{l, n_{l},}\right), e^{\left.-\left(t_{l, q, 1}\right), \ldots, e^{-}\left(t_{l, q, n_{l q}}\right)\right)}\right.
\end{aligned}
$$

From 5-35, the upper bound on $T_{c}$ can be derived. Noting that the execution times of client tasks from $t_{2,0} \ldots, t_{L}, 0$ already appear in the completion time for the layers, we get:

$$
\begin{aligned}
& T_{c}^{+}=\sum_{l=1}^{L} T_{c l^{+}} \cdot T_{0} \text { and } \\
& T_{c}^{+}=\sum_{l=1}^{L} T_{c l} \cdot T_{0}
\end{aligned}
$$

Where

$$
T_{0}=\sum_{l=2}^{L} c\left(t_{l, 0}\right)
$$

From 5-35 and 5-37, we get

$$
\begin{aligned}
T_{c}^{+}= & \sum_{l=1}^{L}\left(c\left(t_{l, 0}\right)+c\left(t_{l+1,0}\right)+\sum_{m=1}^{n_{l}} e^{+}\left(t_{l, m}\right)\right. \\
& \left.+\sum_{q=1}^{Q_{1}} b_{l q} \sum_{i=1}^{n_{l,}} e^{+}\left(t_{l, q, i}\right)\right)-\sum_{l=2}^{L} c\left(t_{l, 0}\right)
\end{aligned}
$$

And from 5-36 and 5-37, the lower bound on $T_{c}$ can be derived. 


$$
\begin{aligned}
T_{c}= & \sum_{l=1}^{L}\left(c\left(t_{l, 0}\right)+c\left(t_{l+1,0}\right)\right. \\
& +\sum_{q=1}^{Q_{l}} b_{l q} \max \left(e^{-}\left(t_{l, 1}\right), e^{\left.\left.-\left(t_{l, 2}\right), \ldots, e^{-}\left(t_{l, n_{l}}\right), e^{-}\left(t_{l, q, 1}\right), \ldots, e^{-}\left(t_{l, q, n_{l q}}\right)\right)\right)}\right. \\
& -\sum_{l=2}^{L} c\left(t_{l, 0}\right)
\end{aligned}
$$

\subsection{Bounds on System with Simple Loops}

Consider a system with a simple loop construct as shown in Figure 5.6. $t_{i}$ is a client task in a precedence graph and may make requests to servers. In this system, each time $t_{i}$ completes execution, either the system terminates itself with probability $1-p_{l}$ or another iteration of the loop is started with the loop probability $p_{l}$. Let $\tau$ denote the expected execution time for the system. Thus,.

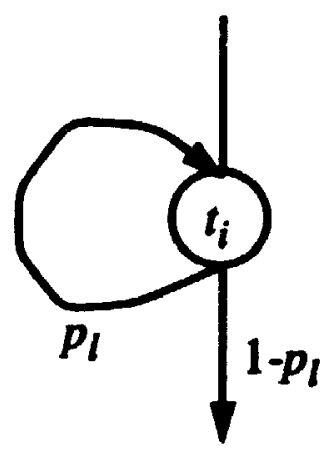

Figure 5.6. General loop

$\tau=e\left(t_{i}\right)+p_{l} \tau$ therefore,

$\tau=e\left(t_{i}\right) /\left(1-p_{l}\right)$ 
If bounds on $e\left(t_{i}\right)$ are known, $e^{-}\left(t_{i}\right) \leq e\left(t_{i}\right) \leq e^{+}\left(t_{i}\right)$, the bounds on $\tau$ are computed in the follnwing manner.

$$
\tau^{+}=e^{+}\left(i_{i}\right) /\left(1-p_{l}\right)
$$

and

$$
\tau^{-}=e^{-}\left(t_{i}\right) /\left(1-p_{l}\right)
$$

Consider, for example, the system stown in Figure 5.7. In this system, the server submodel may include multiple servers. Client task $t_{0}$ and $t_{n+1}$ have no requests to servers and client tasks from $t_{l}$ to $t_{n}$ may make service request to servers. .

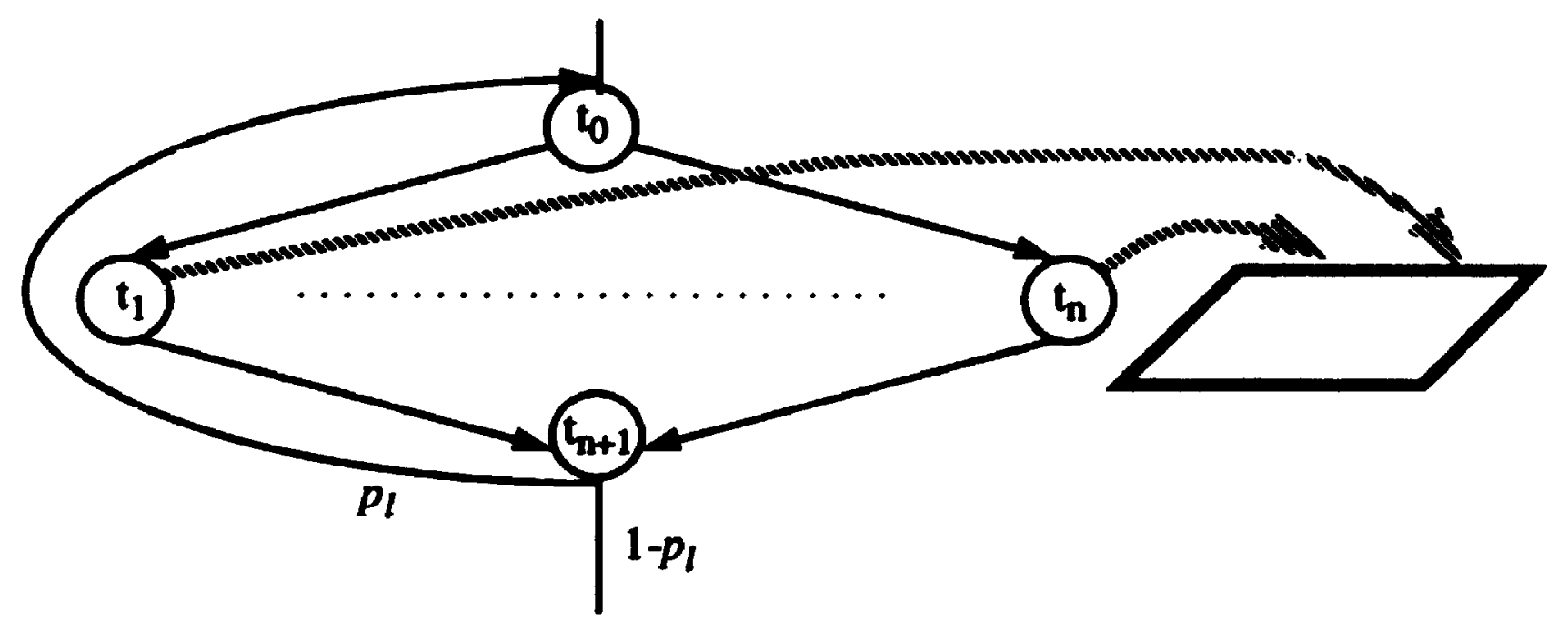

Client submodel

Server submodel

Figure 5.7. Loop system

Equation 5-15 and 5-20 provides the upper bound and lower bound on completion time of 
client system without loop shown in Figure 5.1. The system in Figure 5.7 has loop construct. After the completion of $t_{n+1}$, the execution routing may loop to $t_{0}$ with loop probability $p_{l}$ or terminate with probability $1-p_{l}$. By using 5-40 and 5-41. The upper bounds and lower bounds on $T_{c}$ can be obtained as follows:

$$
\begin{aligned}
& T_{\mathrm{c}} \leq T_{\mathrm{c}}^{+}=\left(\mathrm{c}\left(t_{0}\right)+c\left(t_{n+1}\right)+\sum_{m=1}^{n} e\left(t_{m}\right)\right) /\left(1-p_{l}\right) \\
& T_{c} \geq T_{c}=\left(c\left(t_{0}\right)+c\left(t_{n+1}\right)+\max \left(e\left(t_{1}\right), \ldots, e\left(t_{n}\right)\right)\right) /\left(1-p_{l}\right)
\end{aligned}
$$

\subsection{Computation of the Bounds}

The computations of upper bound and lower bounds are implemented by using a technique based on interval arithmetic as presented in the BNR prolog language [BNR38]. The numerical bounds is examined by the simulation results. A brief description of the interval arithmetic concept and how it is used to compute the bounds are presented.

The concept of interval arithmetic is centered around a data type called interval. An interval is an object that represents a real number which lies between an upper and a lower bound. The bounds of the real number define its range which may vary during the lifetime of a computation.

BNR Prolog [BNR88] developed at Bell Northem Research supplies the interval arithmetic capability as a special feature of the language. The interval arithmetic in BNR Prolog allows us to express arithmetic relationships among intervals and offers a mechanism for solving sets of equations and inequalities. The BNR Prolog interpreter evaluates the set of equations and inequalities and computes the interval which satisfy the set of relationships expressed by equations and inequalities. During the computation, intervals ca. change their values only by narrowing. Both raising the lower bound and lowering the upper bound are called narrowing operation.

The following examples will explain further the interval arithnetic festure of BNR Prolog. The predicate range in the programs sets the initial upper and lower bounds for both the intervals _A and_B. The predicate print_interval is used to print the intervals_A and_B at the end of the computations. The result intervals for_A and _B satisfy the inequalities specified. The interval arithmetic in BNR prolog is explained further with the following examples. 
Exampled (see Figure 5.8)

Brogara:

$\Rightarrow$ ange $(A,[3,5])$.

nge ( $B,(7,9))$,

$A>=B$.

nl, print_interval(_A).

nl, print_interval (_B).

Result:

NO

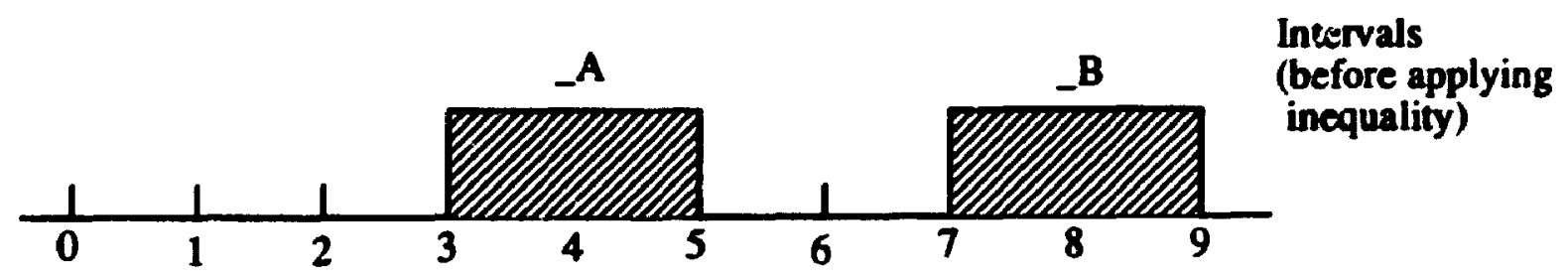

Figure 5.8. Example 1

The result range is NULL ranges, for_A ana _B that satisfy the given constraints can not be found. 
Example 2 (see Figure 5.9)

Brogram:

? range $(A,(1,2))$.

range $\left(\_B,(3,5)\right)$.

${ }_{-} A=<$ B .

nl, print_interval(_A),

nl, print_interval (_B).

Result:

[I.0.2.0001]

[3.0.5.0001]

YES

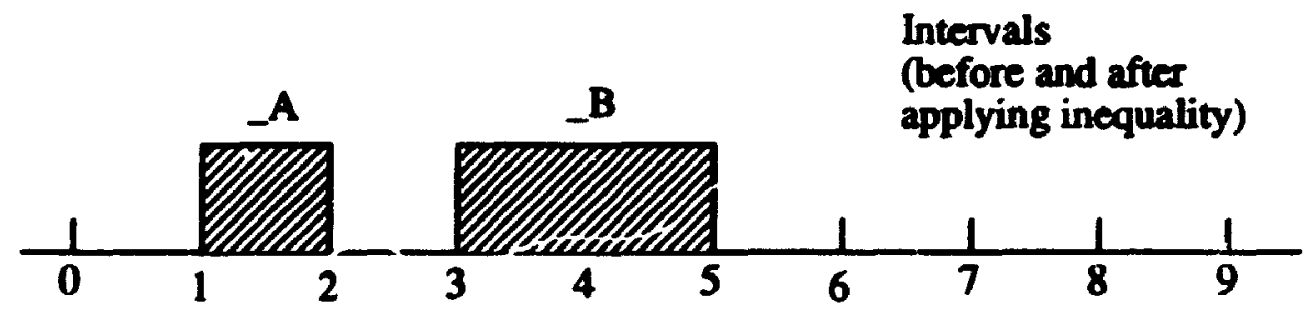

Figure 5.9. Example 2

In this example, no narrowing is performed, because the original intervals satisfy the constrains. 
Example 3 (see Figure 5.10)

Brogram:

? range!_A, $(4,8))$.

range $(B,[6,9))$.

$-A>=$ B .

nl.print_interval(_A),

nl, print_interval (_B).

Besult:

$[6.0,8.0001][6.0,8.0001]$

YES

Intervals

(before applying inequality)
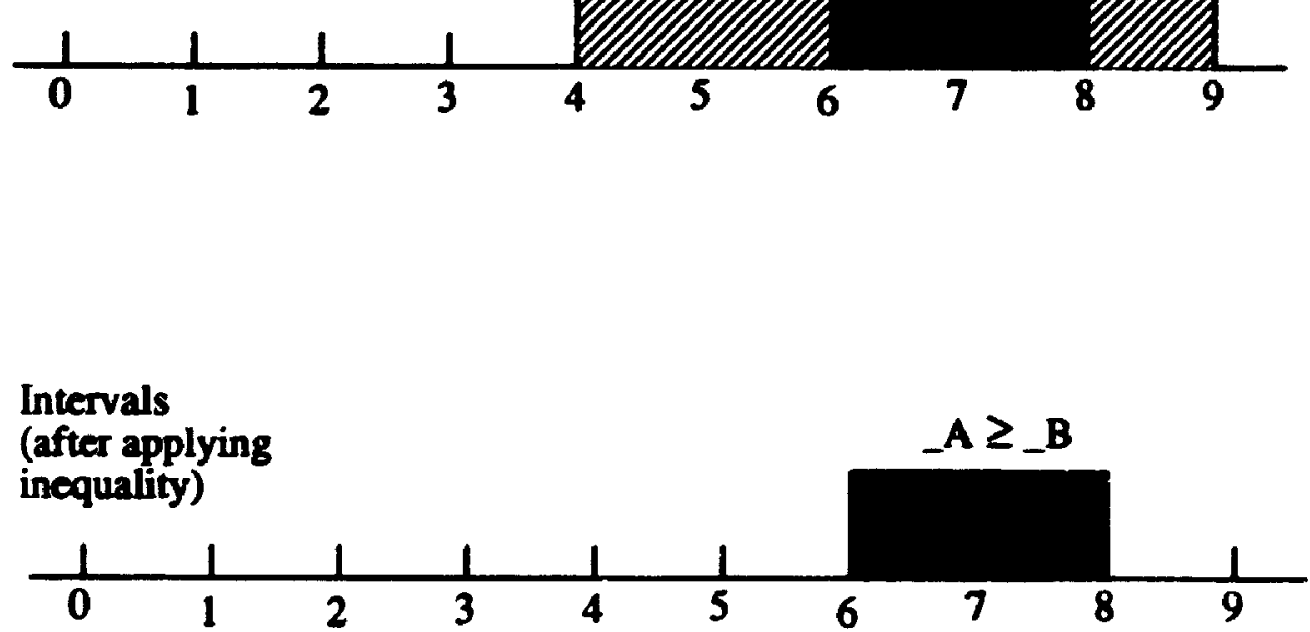

Figure 5.10. Example 3 
Narrowing is performed in this example. In order to satisfy the relationship_A $>=\_B$, the lower bound for _A is raised and the upper bound for _B is lowered.

The interval arithmetic feature of BNR Prolog can be efficiently used for the computation of performance bounds for the RNMTC system. As discussed in the previous section, a system is charixterized by a set of inequalities corresponding to upper and lower bounds. Each inequality corresponding to :aper and lower bounds on parameters such as $e\left(t_{i}\right)$ and $T_{c}$ are converted into a prolog subgoal, when the program executes, each of these subgoals is evaluated and an interval $T_{c}^{-} \leq T_{c} \leq T_{c}^{+}$that satisfies all the inequalities is produced.

The following is an example BNR prolog program used for the system shown in Figure 5.16. The loop probability $p_{l}$ is set at 0.1 . The number of concurrent tasks is 4 .

\section{Program:}

loopntpl :-

9. loopntp1: bound for loop with that the number of concurrent tasks is 4 and loop probability is 0.1 .

räge(_T. $10 . \mathrm{J})$.

${ }_{-} c_{0}$ is 1.0,_ $c_{1}$ is 1.0, $c_{2}$ is 1.0, $c_{3}$ is 1.0, $c_{4}$ is 1.0, $c_{5}$ is 1.0.

\% _ $c_{i}$ is the compusation time for client task $i(i=0, . .5)$

$s_{11}$ is 1.0. $s_{12}$ is 1.0 .

\% _ $s_{11}$ is the service time for first phase of server and $s_{12}$ is the service time for second phase of server

$P_{1}$ is 0.1 .

$-v_{11}$ is 1.0. $v_{21}$ is 1.0. _ $v_{31}$ is 1.0. $v_{41}$ is I.0,

\%_ $v_{i 1}$ is the average number of visits made by client task $i(i=1, .4)$ to server 
$e_{-1}^{*}$ is $\left(c_{l} c_{+} v_{I I}^{*} s_{I I}\right)$.

$e_{2}{ }^{-} i s\left(c_{2}+v_{21}^{*} s_{11}\right)$.

$-e_{3}^{*}$ is $\left(c_{3}+{ }_{2} v_{31}^{*} s_{11}\right)$.

$-e_{4}$ is $\left(c_{4}+\ldots v_{41}^{*} s_{11}\right)$.

\% _ $e_{i}{ }_{i}$ is the lower bound on execution time of client task $i(i=1, \ldots, 4)$

$P_{12 l}$ is $\left(\min \left(I, v_{21} I_{-} v_{l l}\right)\right.$.

$P_{131}$ is $\left(\min \left(I, v_{31}{ }_{-} v_{11}\right)\right.$.

$P_{141}$ is $\left(\min \left(1, v_{41}{ }_{-} v_{11}\right)\right.$.

$P_{2 I I}$ is $\left(\min \left(I, v_{11}{ }_{-} v_{2 l}\right)\right.$.

$P_{231}$ is $\left(\min \left(1, v_{3} I_{-} v_{21}\right)\right.$.

$P_{241}$ is $\left(\min \left(1, v_{41} I_{-} v_{21}\right)\right.$.

$P_{3 I I}$ is $\left(\min \left(1, v_{11}{ }_{-} v_{3 I}\right)\right.$,

$P_{32 I}$ is $\left(\min \left(l, v_{21}{ }_{-} v_{31}\right)\right.$,

$P_{341}$ is $\left(\min \left(I, v_{41} / v_{31}\right)\right.$.

$P_{411}$ is $\left(\min \left(1, v_{11}{ }_{-} v_{41}\right)\right.$.

$P_{421}$ is $\left(\min \left(1, v_{2} J_{-} v_{41}\right)\right.$.

$P_{431}$ is $\left(\min \left(1, v_{31}{ }_{-} v_{41}\right)\right.$, 
\%_ $P_{y I}$ is the probability of client task $t$ being delayed by y at server

$$
\begin{aligned}
& e_{1}{ }^{+} \text {is }\left(_{2} c_{1}+{ }_{-} v_{11} * s_{11}+{ }_{-} v_{11} *{ }_{-} s_{12}+P_{121} *\left(s_{11}+s_{12}\right)+\right. \\
& \left.P_{131} *\left(s_{11}+s_{12}\right)+P_{141} *\left(s_{11}+s_{12}\right)\right) \\
& e_{2}{ }^{+} \text {is }\left(_{-} c_{2}+v_{21} v_{-} s_{11}+{ }_{-} v_{21} * s_{12}+P_{211} *\left(s_{11}+s_{12}\right)+\right. \\
& \left.P_{231} *\left(s_{11}+s_{12}\right)+P_{241} *\left(s_{11}+s_{12}\right)\right) \\
& e_{3}{ }^{+} i s\left(c_{2} 3+v_{31} * s_{11}+\ldots{ }_{31}^{*} s_{12}+P_{311} *\left(s_{1 ;}+s_{12}\right)+\right. \\
& P_{321} *\left(s_{11}+s_{12}, P_{-} P_{341} *\left(s_{11}+s_{12}\right)\right) \\
& e_{4}^{+} i s_{2} c_{4}+v_{41} * s_{11}+v_{41} * s_{12}+P_{411} *\left(s_{11}+s_{12}\right)+ \\
& \left.P_{421} *\left(s_{11}+s_{12}\right)+P_{431} *\left(s_{11}+s_{12}\right)\right)
\end{aligned}
$$

. $e_{\text {min }}$ is (max)_e $\left.e_{1}^{-}, e_{2}^{-}\right)$),

$-e_{\min }$ is (max ( $\left.\left.e_{\min }-e_{3}^{-}\right)\right)$.

$-e_{\min }$ is $\left(\max \left(e_{\min } e_{4}{ }^{-}\right)\right)$.

\% _ $e_{\min }$ is the maximum among _e $e_{1}^{-} e_{2}{ }^{*} e_{3}$ and $e_{4}^{*}$

$$
\begin{aligned}
& -T>=\left(c_{-} c^{+}+c_{5}+e_{\min }\right) /\left(I-P_{l}\right), \\
& T=\left\langle\left(c_{0} c_{-} c_{5}+e_{2}^{+}+e_{3}^{+}+e_{4}^{+}+e_{5}^{+}\right) /\left(1-P_{l}\right),\right.
\end{aligned}
$$

nl, print_interval(_T).

result:

$[4.4444,42.223]$

YES 


\subsection{Test Cases}

A number of test cases have been studied. The computation of the bo:nds is realized by using BNR prolog and the bounds are compared with simulation results. The duration of simulation is set sufficiently long to ensure a confidence interval of the order of $\pm 1 \%$ at a confidence level of $99 \%$. The major motivation for the test cases is the investigation of the BNR prolog implementation of the bound computations. A detailed study of the tightness of the bounds is beyond the scope of the thesis.

\subsubsection{Deterministic System}

A deterministic system is shown in Figure 5.11.

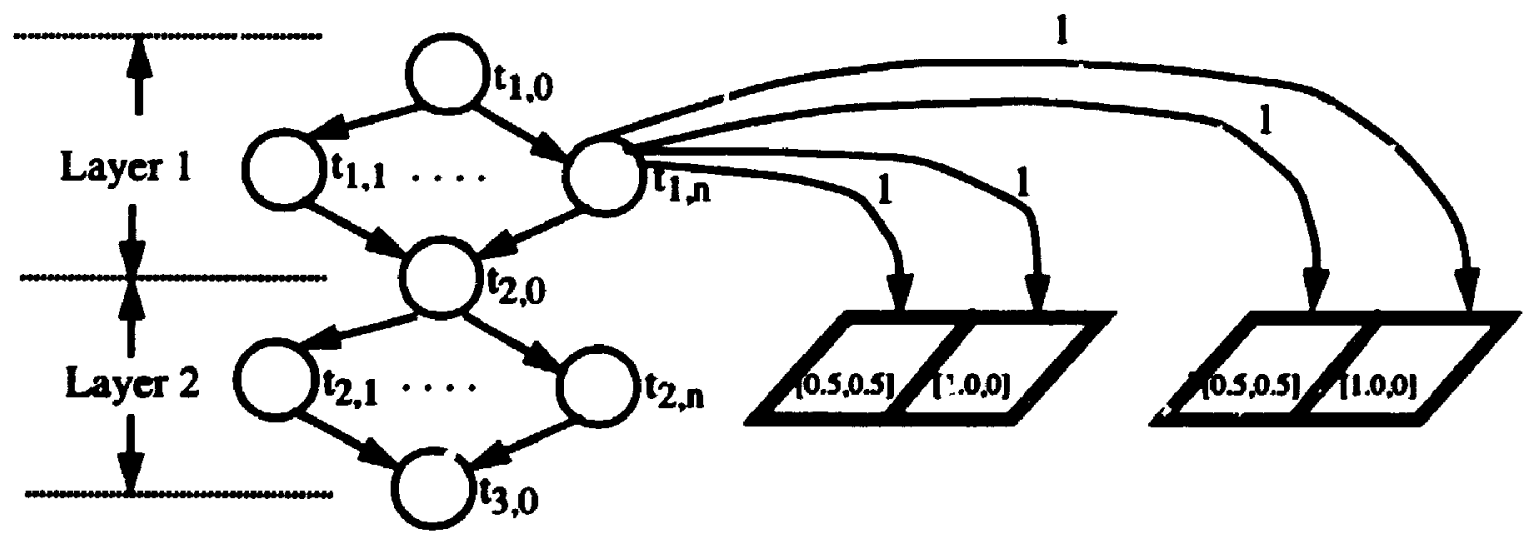

Client submodel

Server submodel

Figure 5.11. Test case of multiple layer system 
There are 2 layers of client tasks. The computation time for all client tasks is 1.0. Client tasks from $t_{1,1}$ to $t_{1, n}$ and from $t_{2,1}$ to $t_{2 \Omega}$ make a single service request to each entry in all the servers. There are $n$ concurrent client tasks in each layer. The parameters for servers are shown in Figure 5.11. In each case, three different values of $n,(n=1,4,8)$ are experimented.

- Fixed computation time and service time

In this case, the computation time for client tasks and service time for servers are fixed. Figure 5.12 shows the bounds, for the sake of comparison the simulation results are included. It is interesting to note that the comf'etion time $T_{c}$ obtained from the simulating is approximately in the middle of wounds $T_{c}^{+}$and $T_{c}^{-}$.

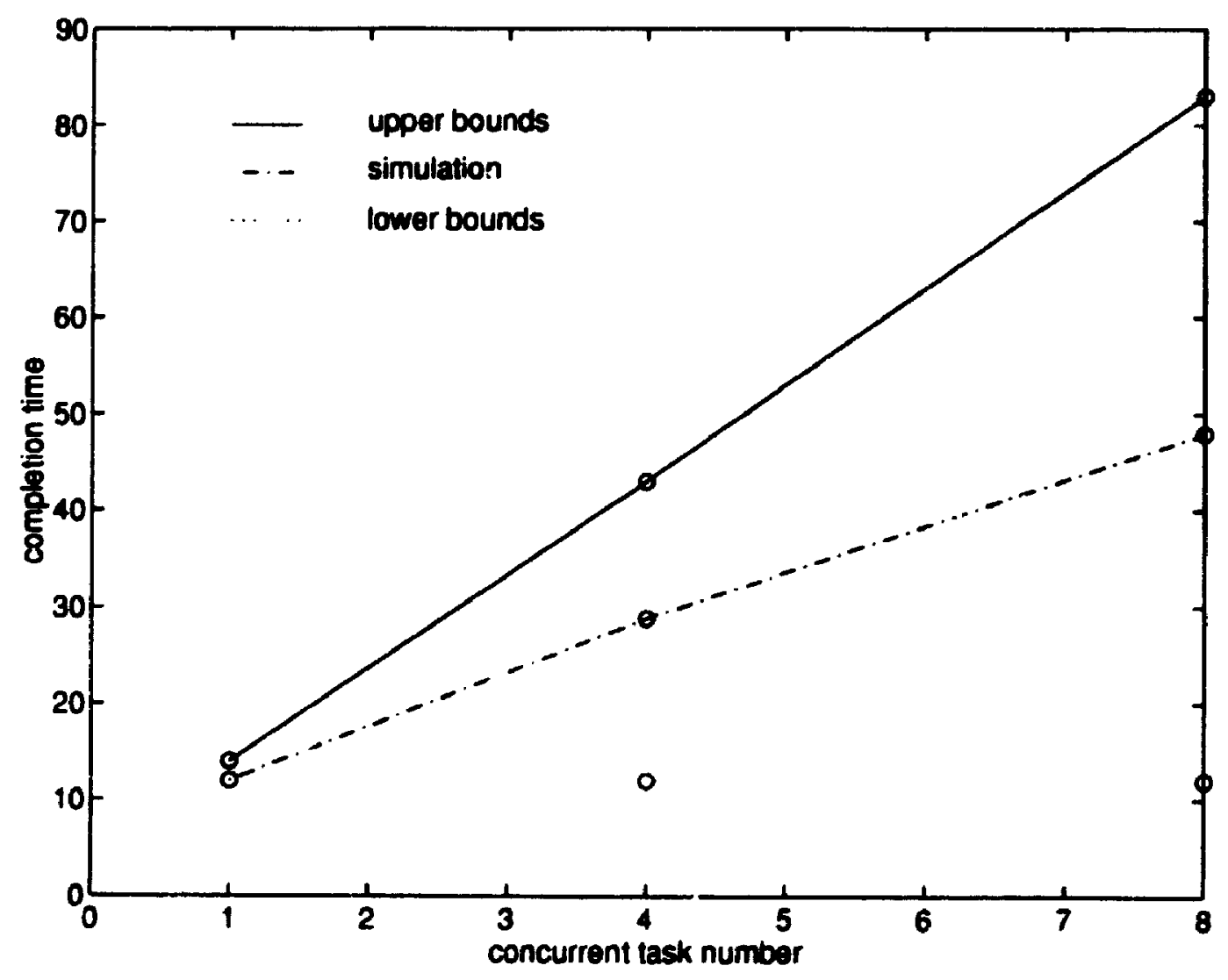

Figure 5.12 Test result for system with fixed computation time and service time 
- Exponential distribution of computation and service time

The system shown in Figure 5.11, with exponential distribution of computation and service time is investigated. Figure 5.13 shows the result for this case. the lower bound seems to be closer to the mean completion time in comparison to the upper bound.

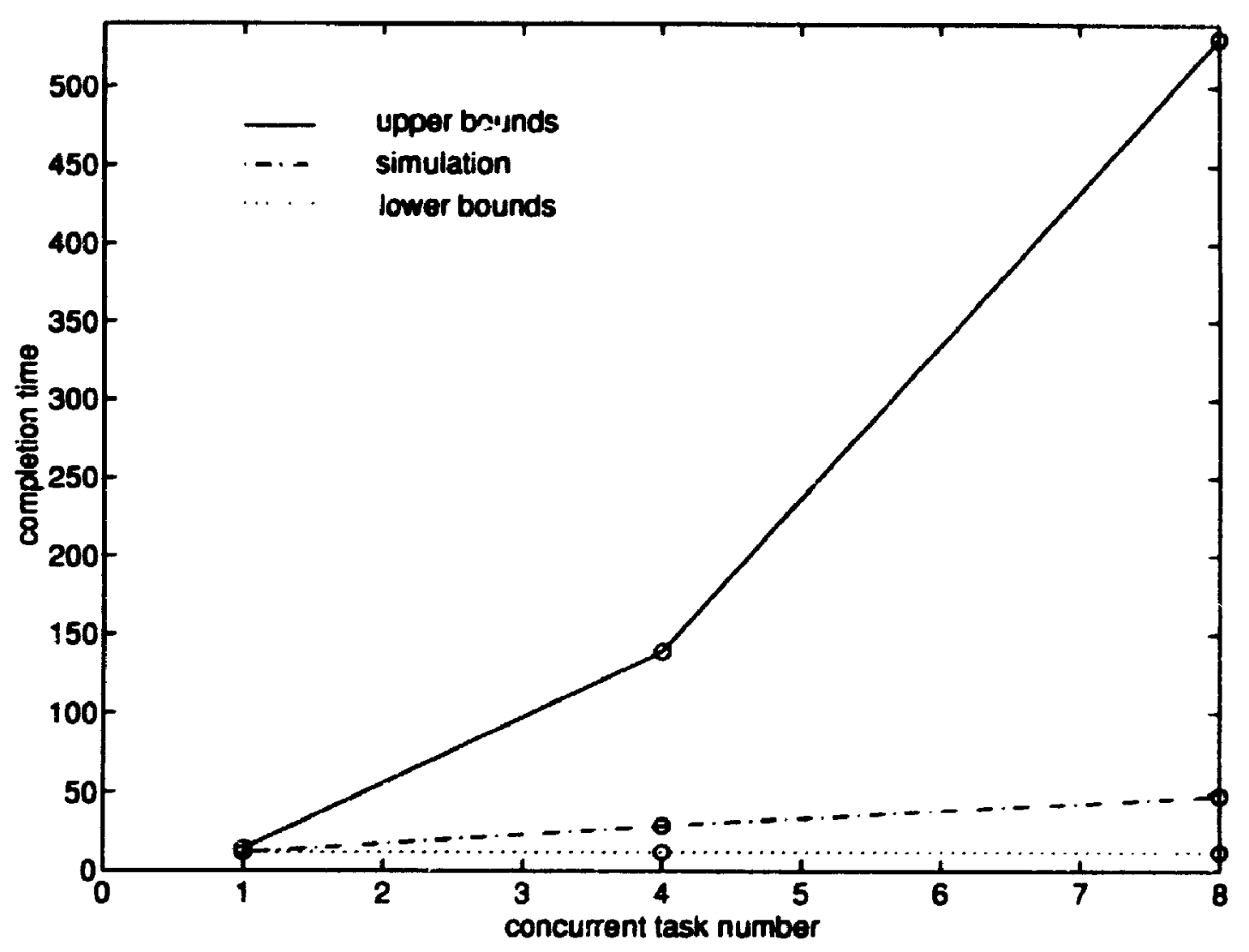

Figure 5.13. Test case for exponential distribution 


\subsubsection{Probabilistic Branching}

A system with probabilistic branching shown in Figure 5.14 is tested.

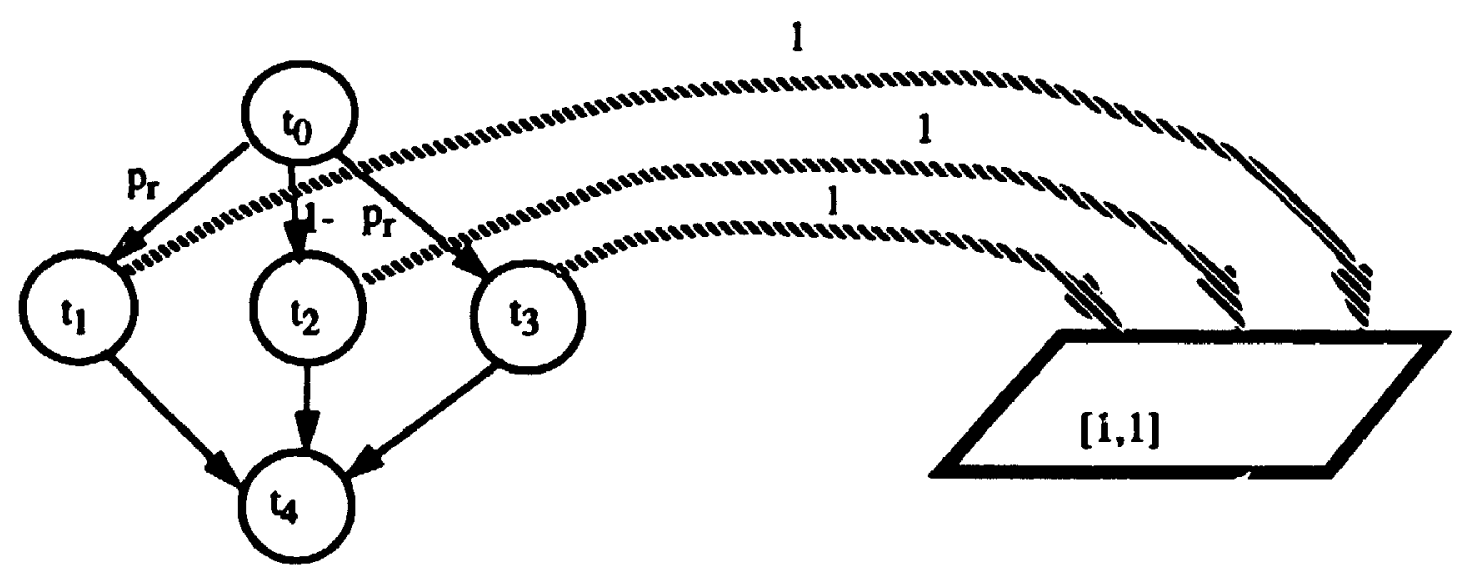

Figure 5.14. Test case of probabilistic branching

Th.ere is a single server called by $t_{1}, t_{2}$ and $t_{3}$ with number of visits made to the server by each client task is 1 . Client task $t_{3}$ is ari $A N D$ class task and starts execution upon the completion of $t_{0}$. Client tasks $t_{1}$ and $t_{2}$ are OR class tasks. After the termination of $t_{0}$, either $t_{1}$ starts or $t_{2}$ starts. Client tasks $t_{0}$ and $t_{4}$ have no service requests to server. The computation times for client task $\ell_{2}$ is set 5.0 whereas the computation times for all of other client tasks are set 1.0. The parameters for the server is shown in Figure 5.14.

The results are shown in Figure 5.15. The lower bound seems to be tighter than the upper 
bound.

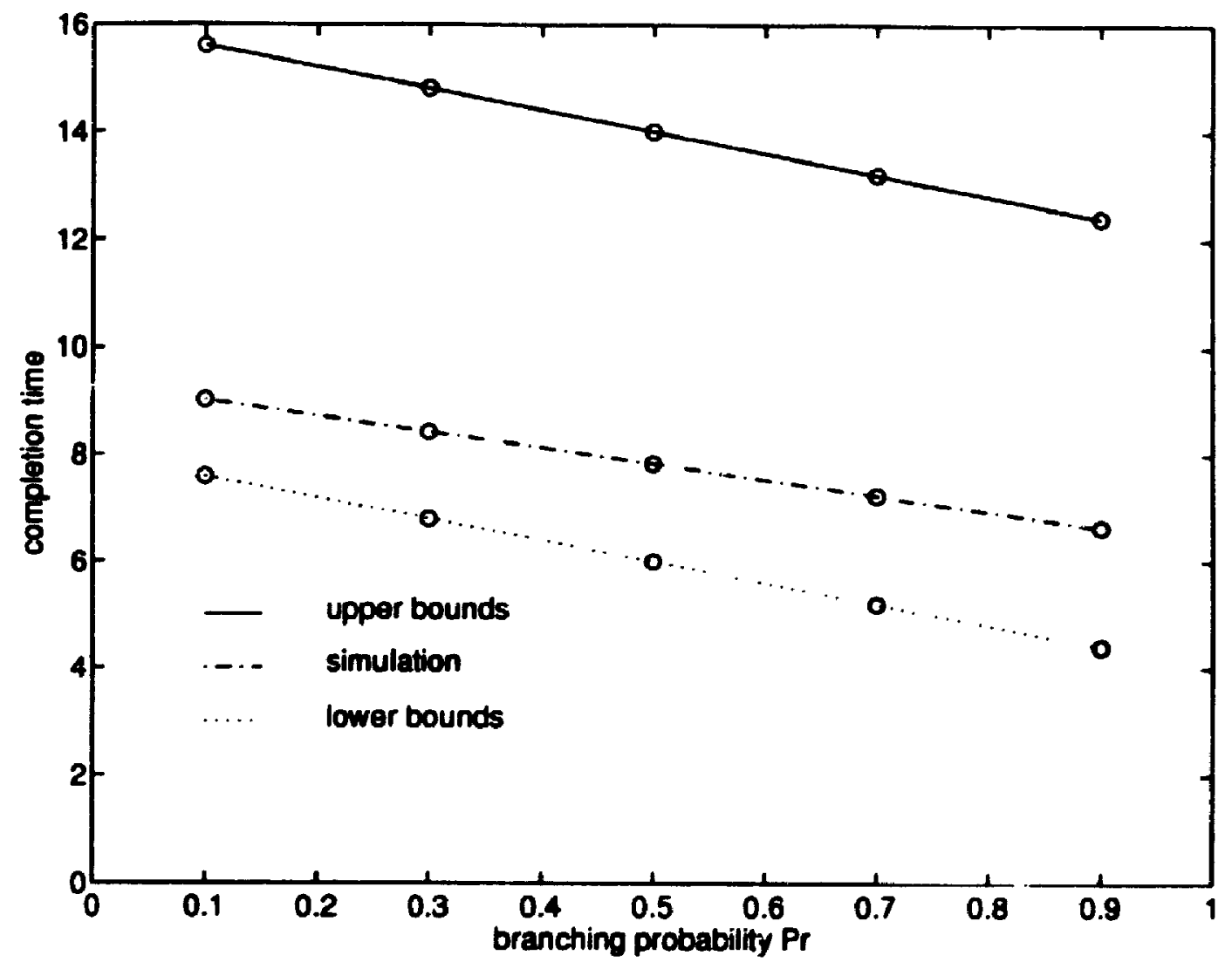

Figure 5.15. Test result of probabilistic branching 


\subsubsection{System with Loop}

A test case for a system with a single loop is presented next. The system is shown in Figure S.16.

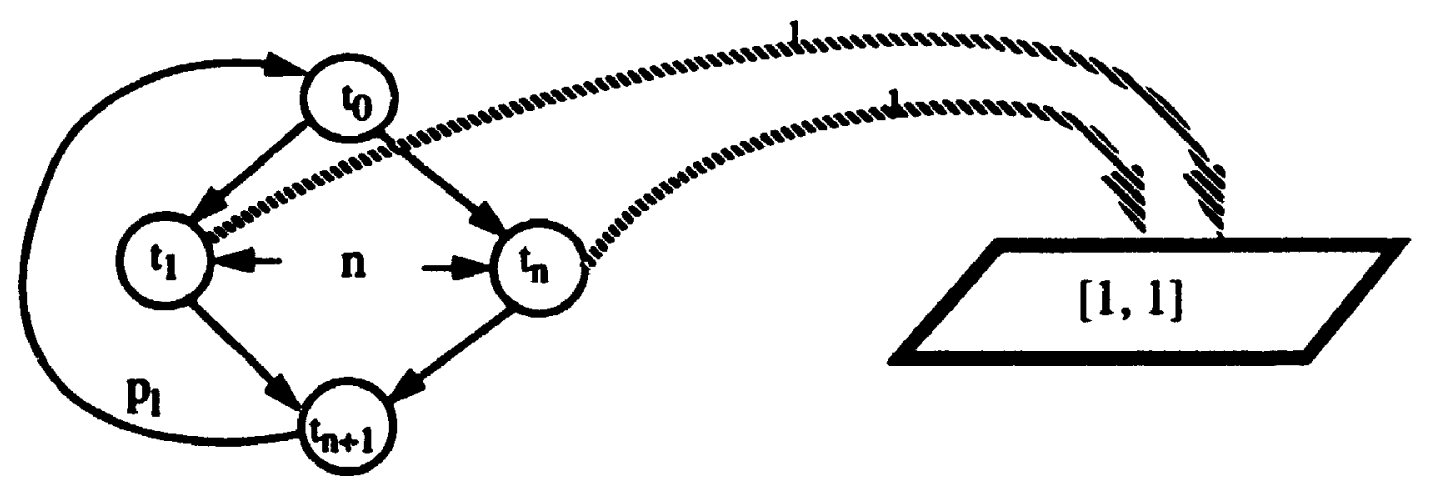

Figure 5.16. Test case of loop system

Client task $t_{0}$ and $t_{n+1}$ have no requests to server while other client tasks may make requests to the server and the mean number of visit to the server is 1.0. The computation times and service time are exponentiai distributed. The computation times for all client tasks are 1.0. The parameters for server client is given in Figure 5.16. After the termination of the client task $t_{n+1}$, the system may either loop back to client task $t_{0}$ with a loop probability $p_{l}$ or terminate.

Figure 5.17 shows the results for 4 concurrent client tasks. The test is repeated with $n=8$ 
and the result are presented in Figure 5.18.

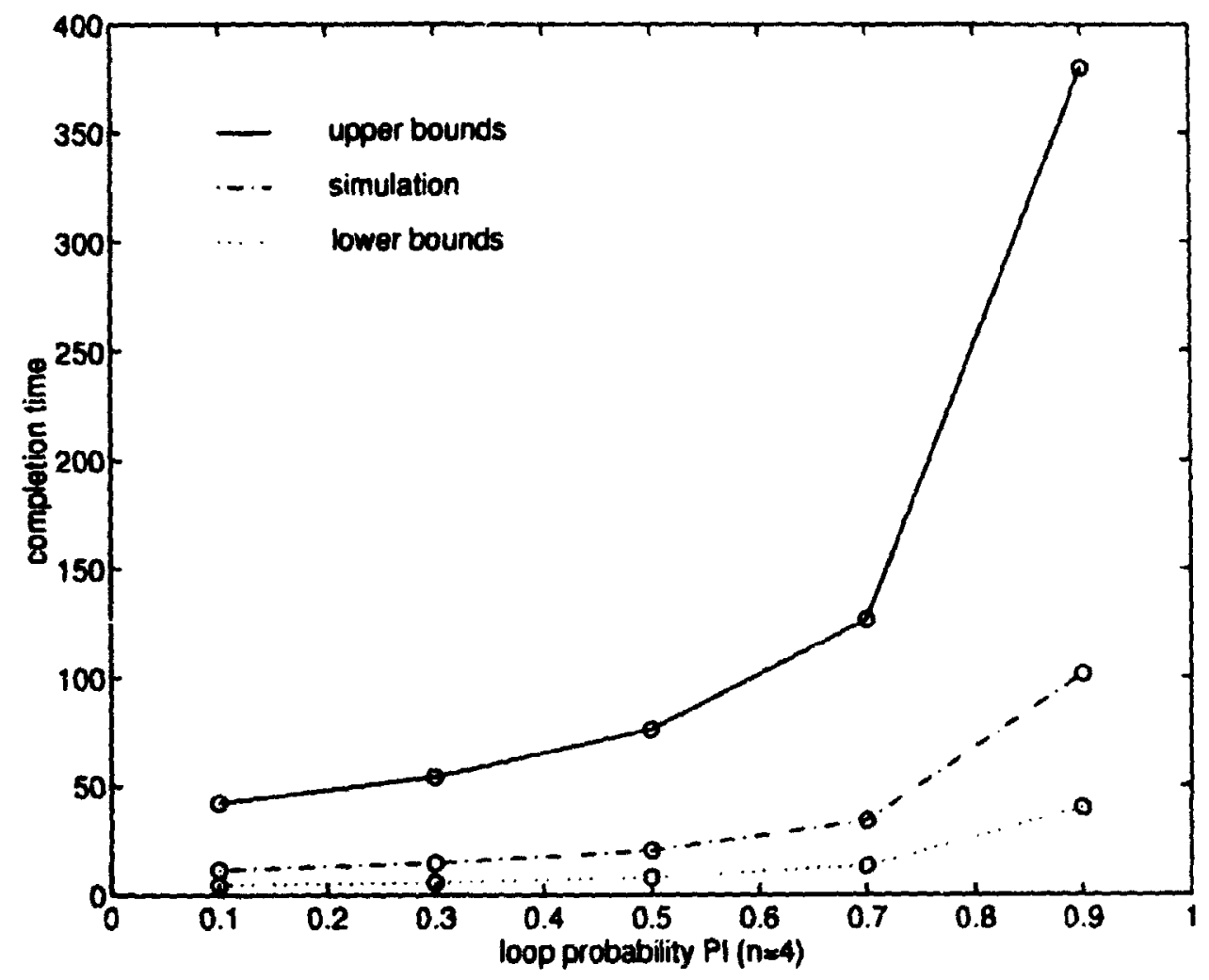

Figure 5.17. Test result for loop with 4 concurrent client tasks 


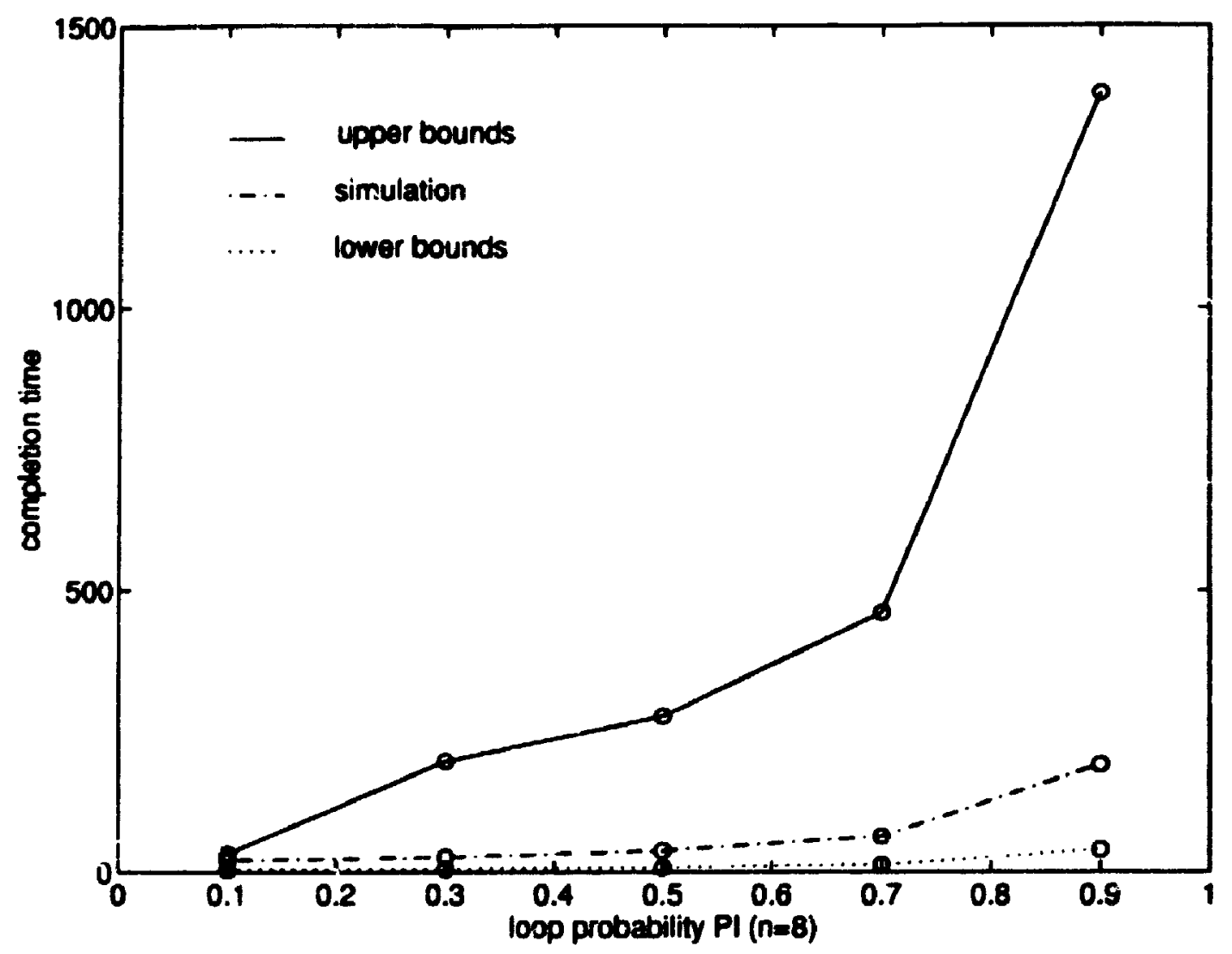

Figure 5.18. Test result for loop with 8 concurrent client tasks

For both cases the lower bound seems to be quite tight. The tightness seems looser with the increasing of $p_{l}$. 


\section{Chapter 6}

\section{Test Cases for Analytic Solution Method}

RNMTC model have been used with a number of different test cases and the result of the approximate analysis for each case is compared with that of simulation experiments. Both simpler test cases and a large transaction processing system are analyzed. The simulation experiments were run for sufficiently long durations of time to yield a confidence interval of the order of $\pm 1 \%$ at a confidence level of $99 \%$. The error rate $\varepsilon$ is captured according to the following computation:

$$
\varepsilon=\frac{\text { outpL. of analytic solver }- \text { simulation result }}{\text { simulation result }}
$$

The performance measures derived from the analytic solver reside in reasonable range. The results show that the error rates for most of test cases are less than 10\%. Section 6.1 shows the simpler cases and the results, and section 6.2 is the analysis of large transaction processing tandem database system.

This chapter is organized as the following way: section 6.1 gives the general test cases, such as balanced system, impact of the number of concurrent tasks, impact of layer of client tasks, unbalanced systen, loops, probabilistic branching and processor contention. Section 6.2 presents a large RNMTC model of a distributed database system for two classes of client queries. The identify of bottleneck is also studied.

\subsection{General Test Cases}

A set of general test cases have been made to examine the accuracy and efficiency of the 
approximate analysis for RNMTC sysuem. Each of the test cases is a representation of a specific kind precedence graph, such as, AND, OR, probabilistic tranching and loop, etc.

\subsubsection{Model 1: Balanced System}

Balanced system have balanced precedence graph and SRVN graph. The computation time and service time are also evenly assigned. The balanced system under test is shown in Figure 6.1. All computation time, service time and visit recio from client tasks to server $s_{1}$ and server $s_{2}$ are set 1 , the only variable is the visit ratio from servers $s_{1}$ and $s_{2}$ to server $s_{3}$. Table 1.1 on page 87 shows the error rate $\varepsilon$ between the result from analytic solution method and the result from simulation corresponding to different values of visit ratio. Figure 6.2 presents $\varepsilon$ as a function of the utilization of server $s_{3}$. For the balanced system, the relative error seems to be low for very low and very high values of $s_{3}$ utilization whereas the highest \& $(\varepsilon=-8.273)$ is obtained at an intermediate value of $s_{3}$ utilization.

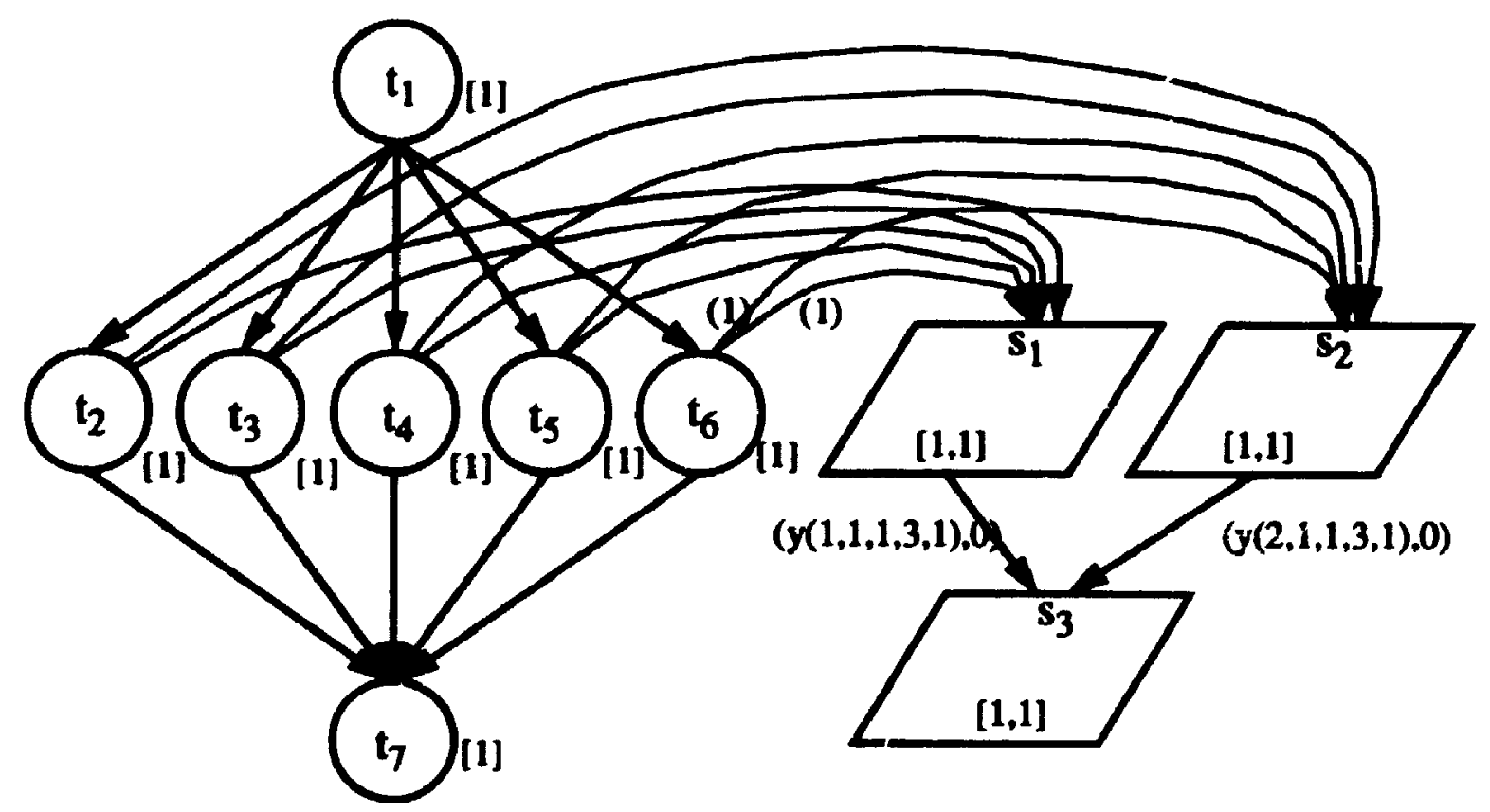

Figure 6.1. Balanced sysiem 


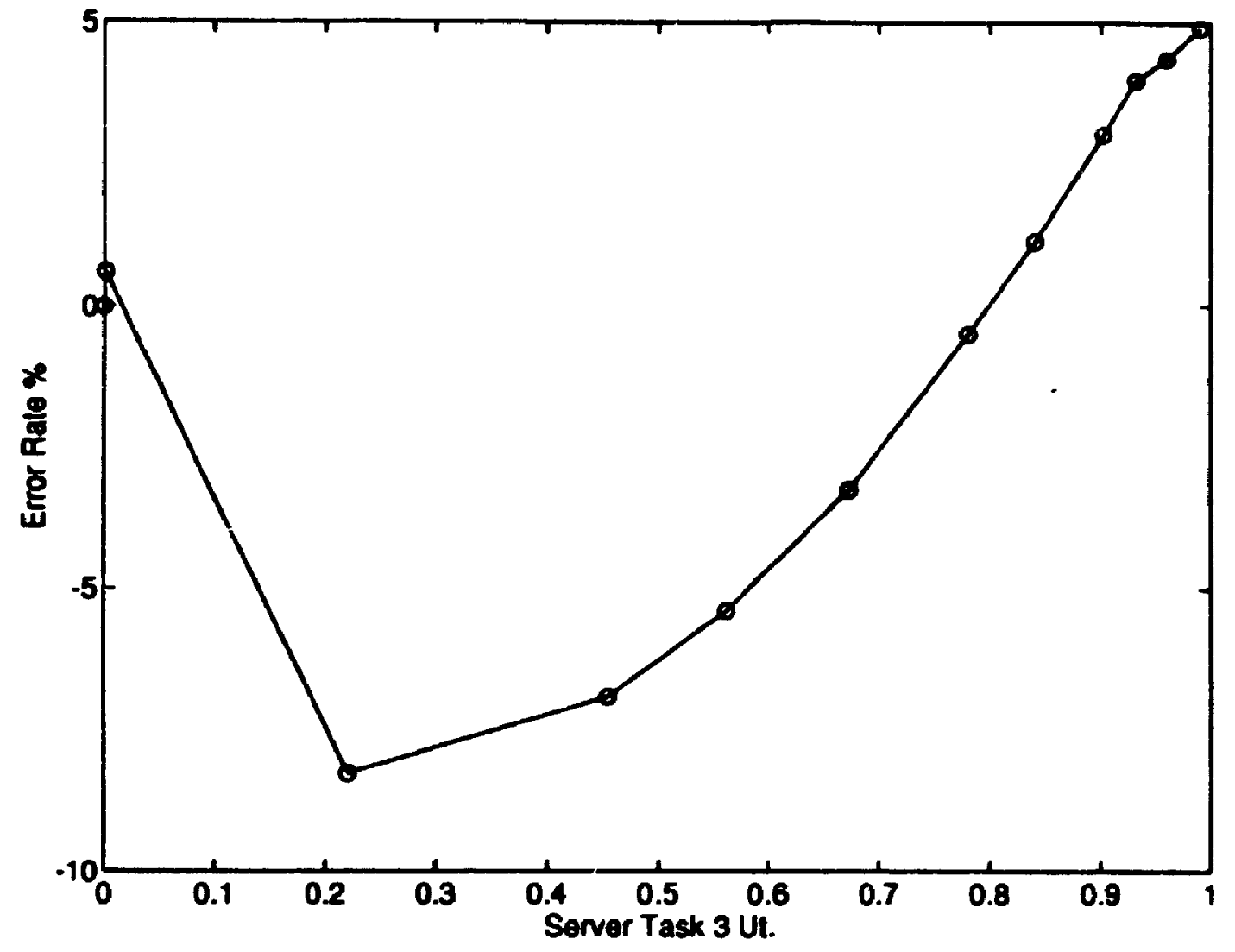

Figure 6.2. Error rate for balanced system

\subsubsection{Model 2: Impact of the Number of Concurrent Tasks}

In this section, a simple concurrent system is analyzed. The concurrence of the system is 
increased by forking new concurrent tasks. Figure 6.3 is a RN. ITC model for such a system. The analytic solution is applied to the system with different number of concurrent tasks. All the computation times and service time are set ! while visit ratios from client tasks to the server $v(i, 1,1,1)$ $(i=1, \ldots, n)$ is a variable.

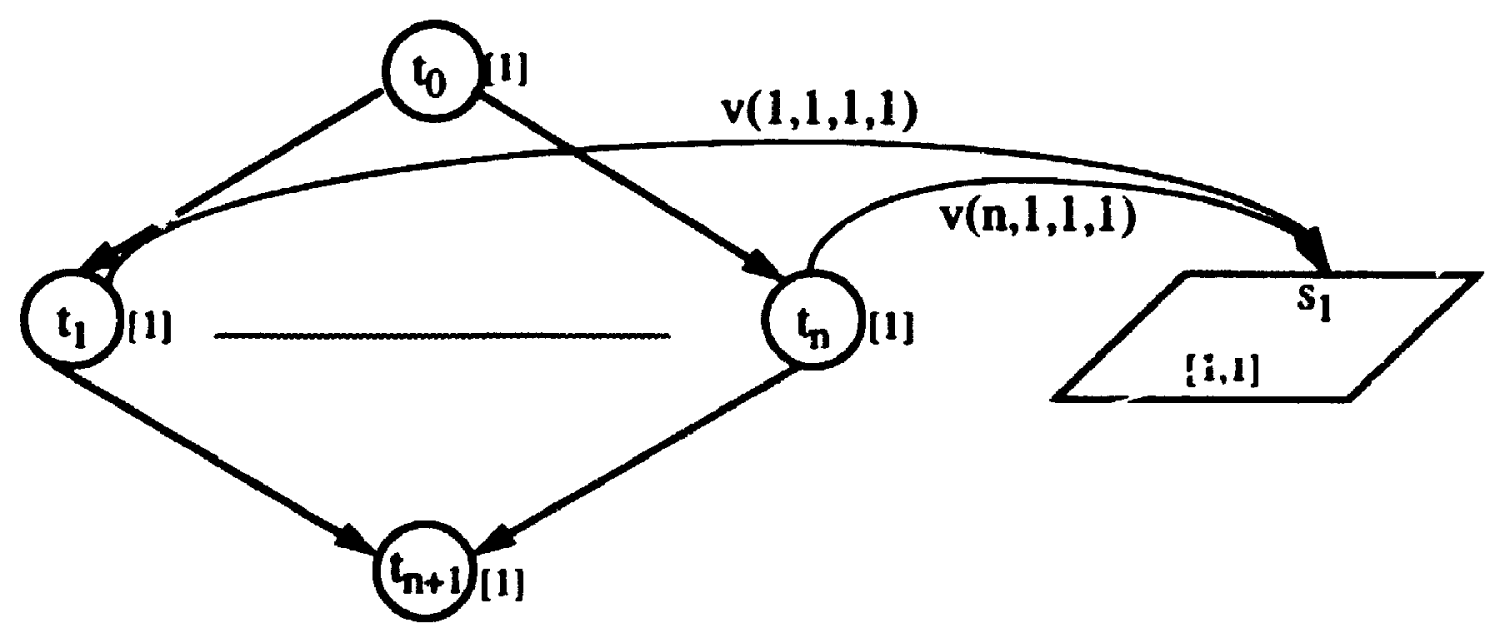

Figure 6.3. Impact of number of concurrent tasks

The experiments were repeated with different number of concurrent tasks. Table 2.1 on page 87. Table 2.2 on page 88 and Table 2.3 on page 89 give the numerical results for client cycle time from analytic solution and simulation and the error rate. The error rate is insensitive with the number of concurrent tasks for large range of variation of visit ratio.

\subsubsection{Model 3: Impact of layer of Client Tasks}

In this test case, the impact of multiple layers of join-fork is investigated. The client tasks in 
each layer have the same structures and parameters. All concurrent tasks $t_{i j}(i=1, \ldots, n ; j=1, \ldots .5)$ call servers $s_{1}$ and $s_{2}$ with visit ratio is set 1 . Execution time for client tasks and service time for server $s_{i}(i=1,2,3)$ in both first phase and second phase are set 1. Figure 6.4 is the structure of the system. Table 3.1 on page 89 shows the numerical error rate for different number of layers. From the result, one can see that the error rate is fairly insensitive to the number of join-fork layers.

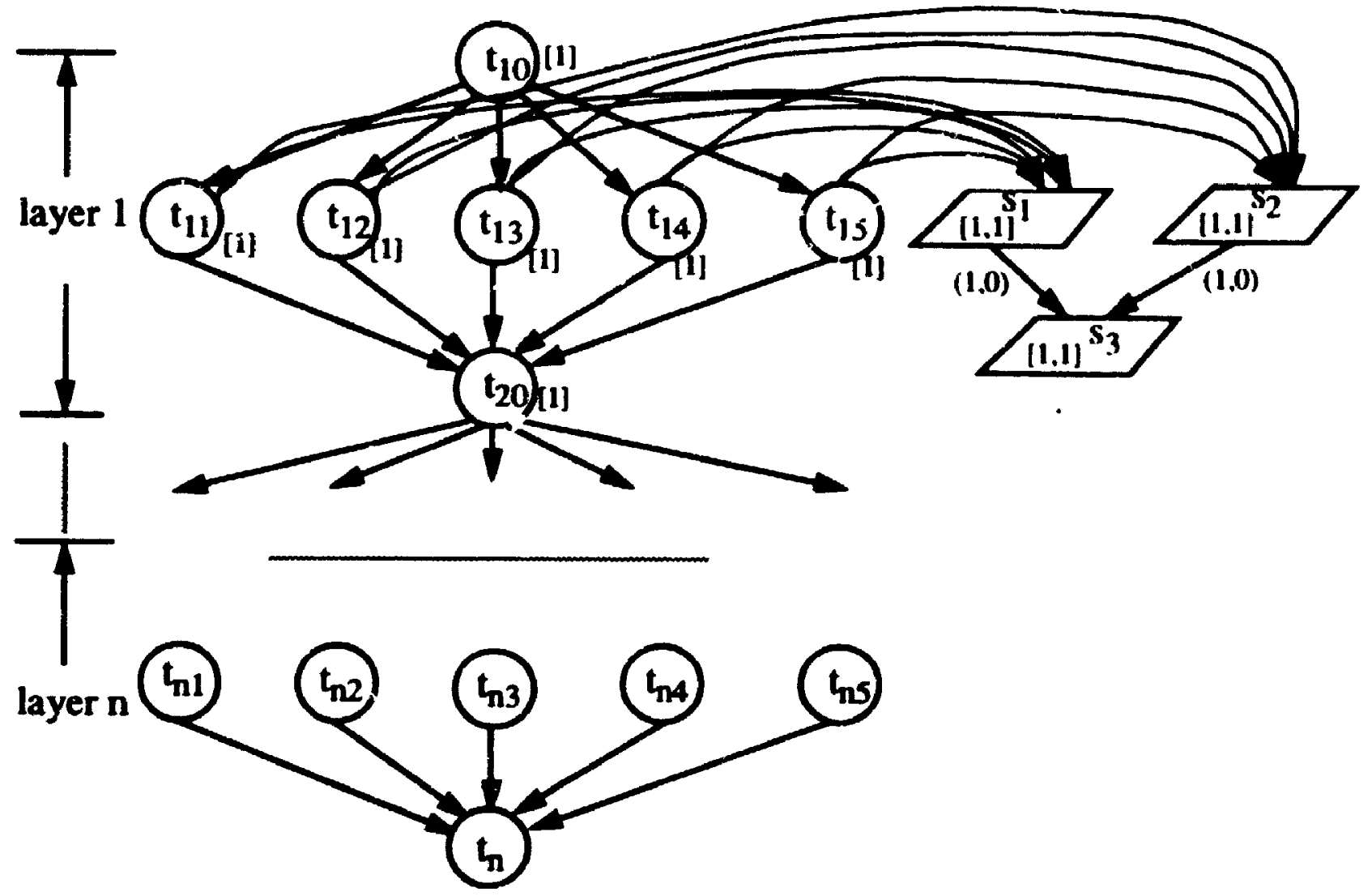

Figure 6.4. Variation of rlient task layers

\subsubsection{Model 4: Unbalanced System}

The test cases in section 6.1.2 and 6.1.3 are generally balanced. This section tests an unbal- 

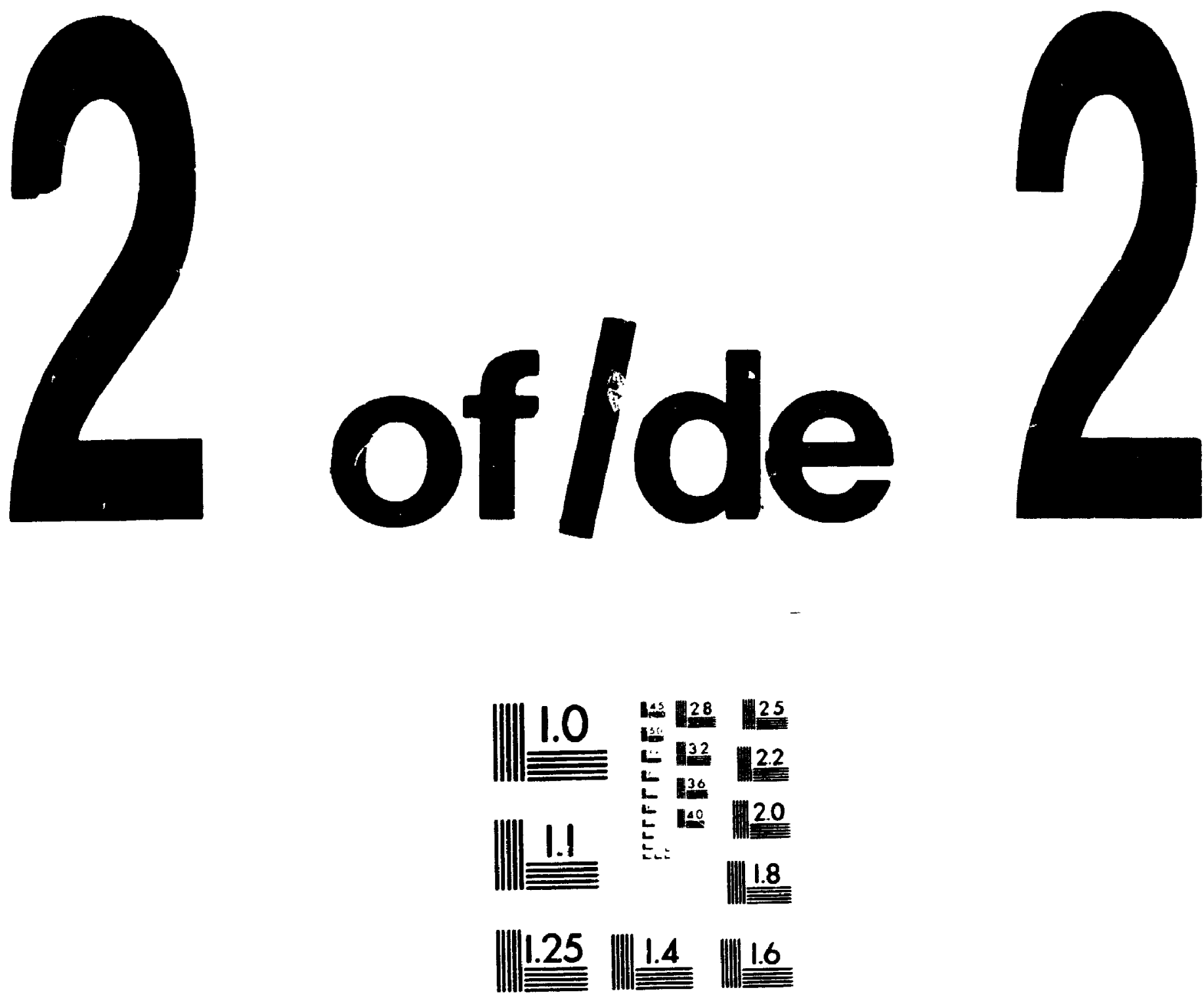

MICROCOPY RESOLUTION TEST CHART

NATIONAL BUAEAU OF STANDARDS

STANDARD REFERENCE MATERIAL 1010a

(ANSI ald ISSI TEST CHART NO 2) 
anced system shown in Figure 6.5. The computation time. service time and visit ratio are unevenly distributed. The parameters are shown in Figure 6.5.

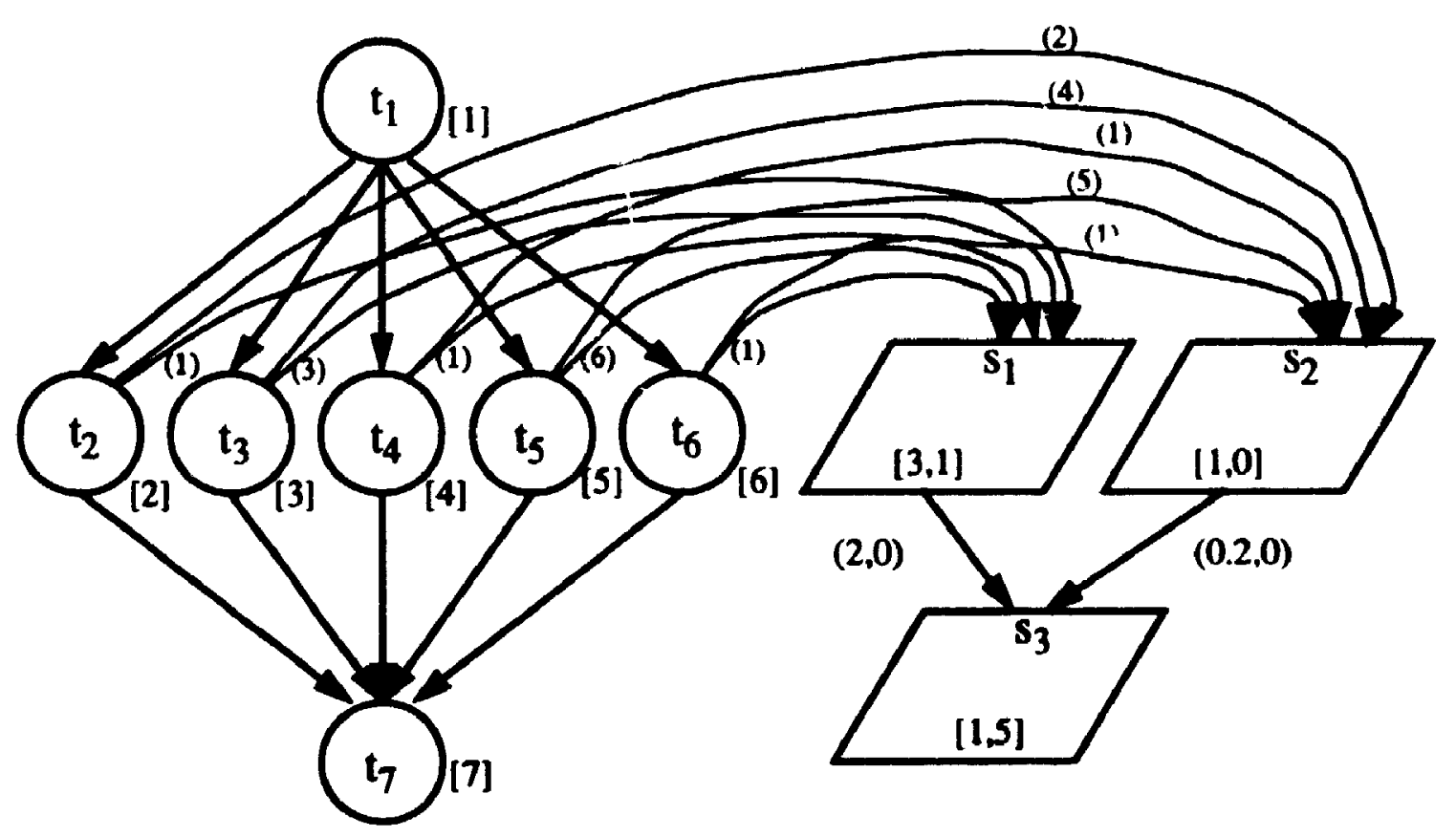

Figure 6.5. Unbalanced System

The mean cycle time of the client is $\mathbf{1 7 5 . 3 8}$ for analytic solution methods while it is 171.822 for simulation. The error rate is low with value $2.08 \%$.

\subsubsection{Model 5: Loops}


Figure 6.6 is a system with loop. After the termination of client task $t_{8}$, either $t_{9}$ is enabled with probability 0.1 or $t_{2}$ is initiated again with loop probability 0.9 . All parameters are given in Figure 6.6.

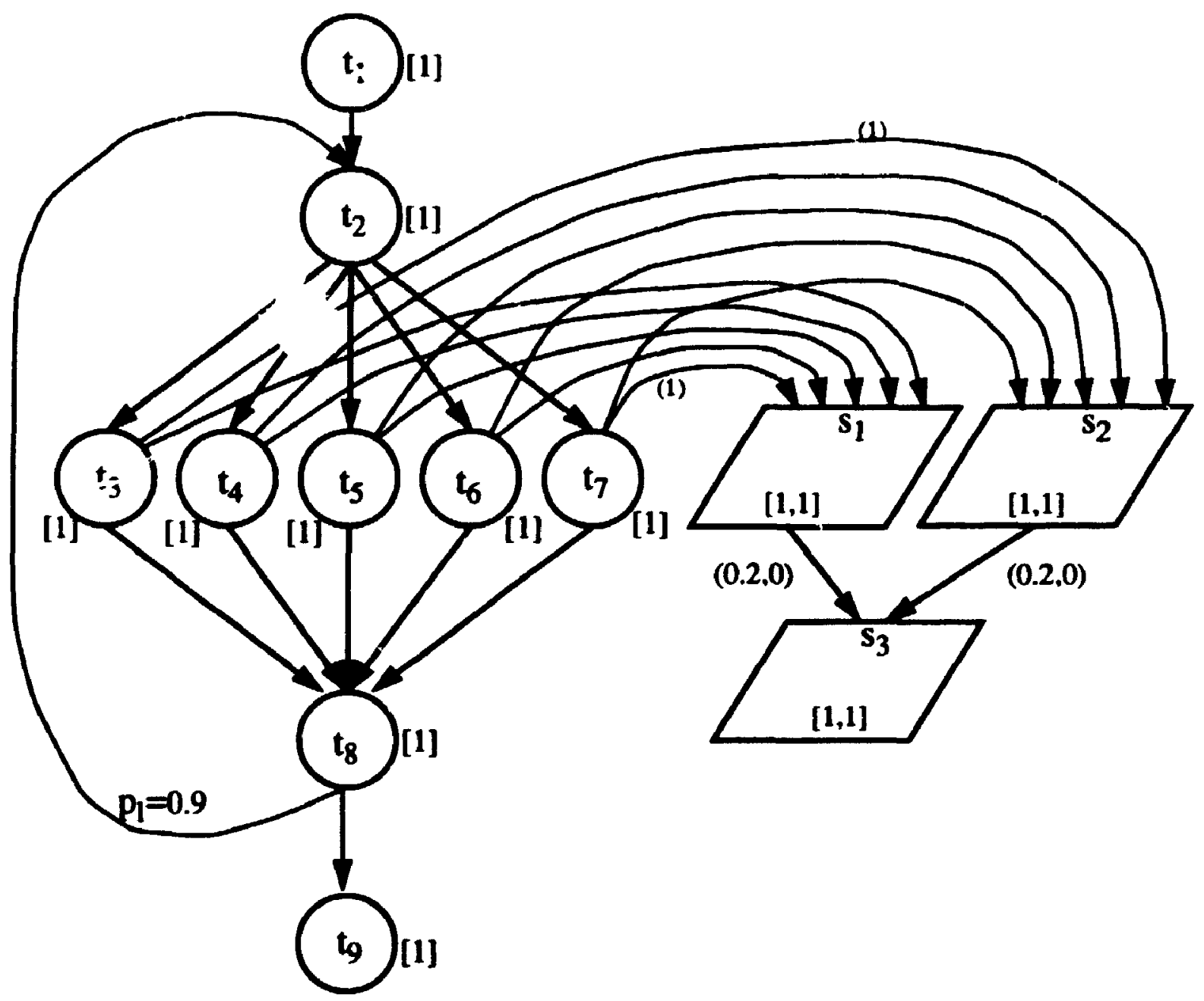

Figure 6.6. Loops

The mean cycle time is $\mathbf{1 6 8 . 3 8}$ and $\mathbf{1 8 3 . 6 6}$ for analytic solution method and simulation respectively. Entor rate is $-9.07 \%$. 


\subsubsection{Model 6: Probabilistic Branching}

Figure 6.7 is a single thread system with probabilistic branching. After the termination of ciient task $t l$, there are three possible executing routines with different probabilities.

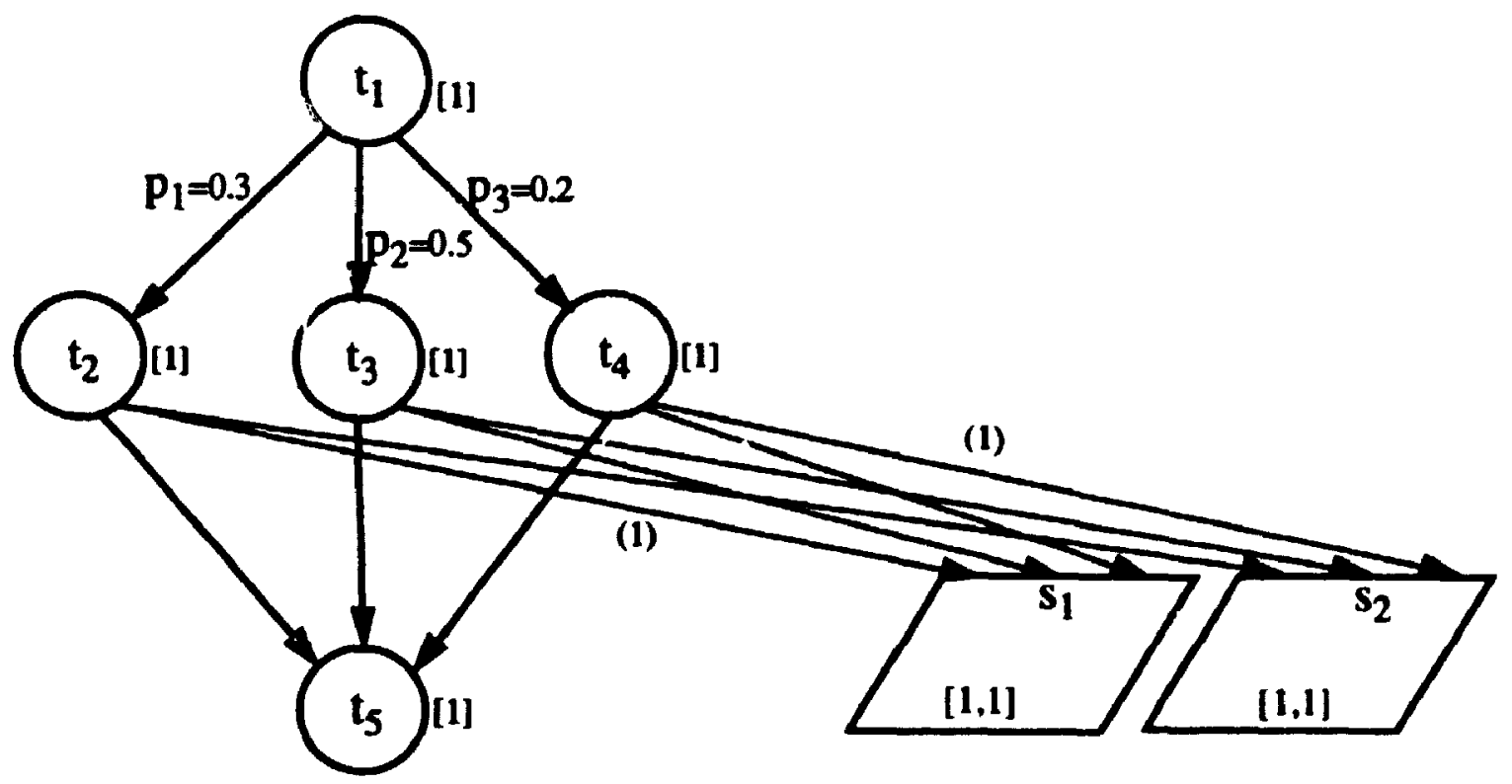

Figure 6.7. Probabilistic branching

The test result shows the error rate is $0.056 \%$ derived from analytic solution method result 5.6055 and simulating result 5.6024 . 


\subsubsection{Model 7: Proc $s$ ssor Contention}

For the above examples, all test cases are based on the assumption that each client task and server has its own processor so that there is no contention for hardware.

This section shows how the analytic solution method can be vsed to evaluate the impact of hardware contention. Figure 6.8 is RNMTC model in which client task $t_{1}, t_{8}$ and server $s_{1}$ have their own processor whereas client task $t_{i}(i=2, \ldots, 7)$ may compete for processors. The number of processors for client task $t_{i}(i=2, \ldots, 7)$ is varied from 1 to 6 . The homugenerous processors are shared equally by $t_{i}(i=2, \ldots, 7)$. For example, if the number of processor is $3, t_{2}$ and $t_{3}, t_{4}$ and $t_{5}, t_{6}$ and $t_{7}$, each pair shares one CPU. As stated in section 2.2, processor can be modeled as a srvn server. The computation time of a client task which is allocated on a processor can be converted to the service time of the processor.

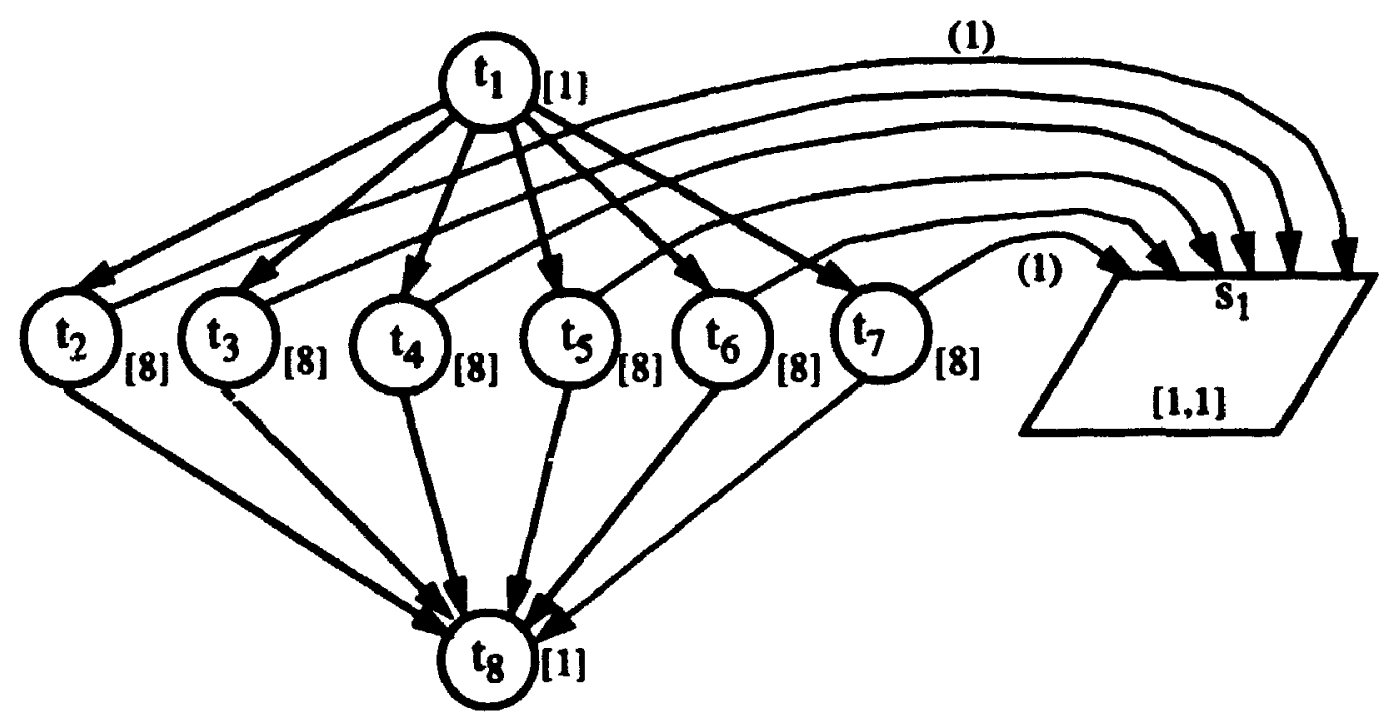

Figure 6.8. Processor contention

Figure 6.9 is a RNMTC model corresponding to Figure 6.8 for 3 processors. The processors $\left(\mathrm{CPU}_{1}, \mathrm{CPU}_{2}\right.$ and $\left.\mathrm{CPU}_{3}\right)$ are viewed as SRVN servers. The computation time of $t_{i}(i=2, \ldots, 7)$ is set 0 because computation time of a client task is converted to service time of the processor which the client task is allocated on. The visit ratio from client task to CPU server is $\mathbf{2}$ due to that total visit ratio from the client task to server is 1 . Table 4.1 on page 90 shows analytic result and simu- 
lation result the error rate is less than $10 \%$.

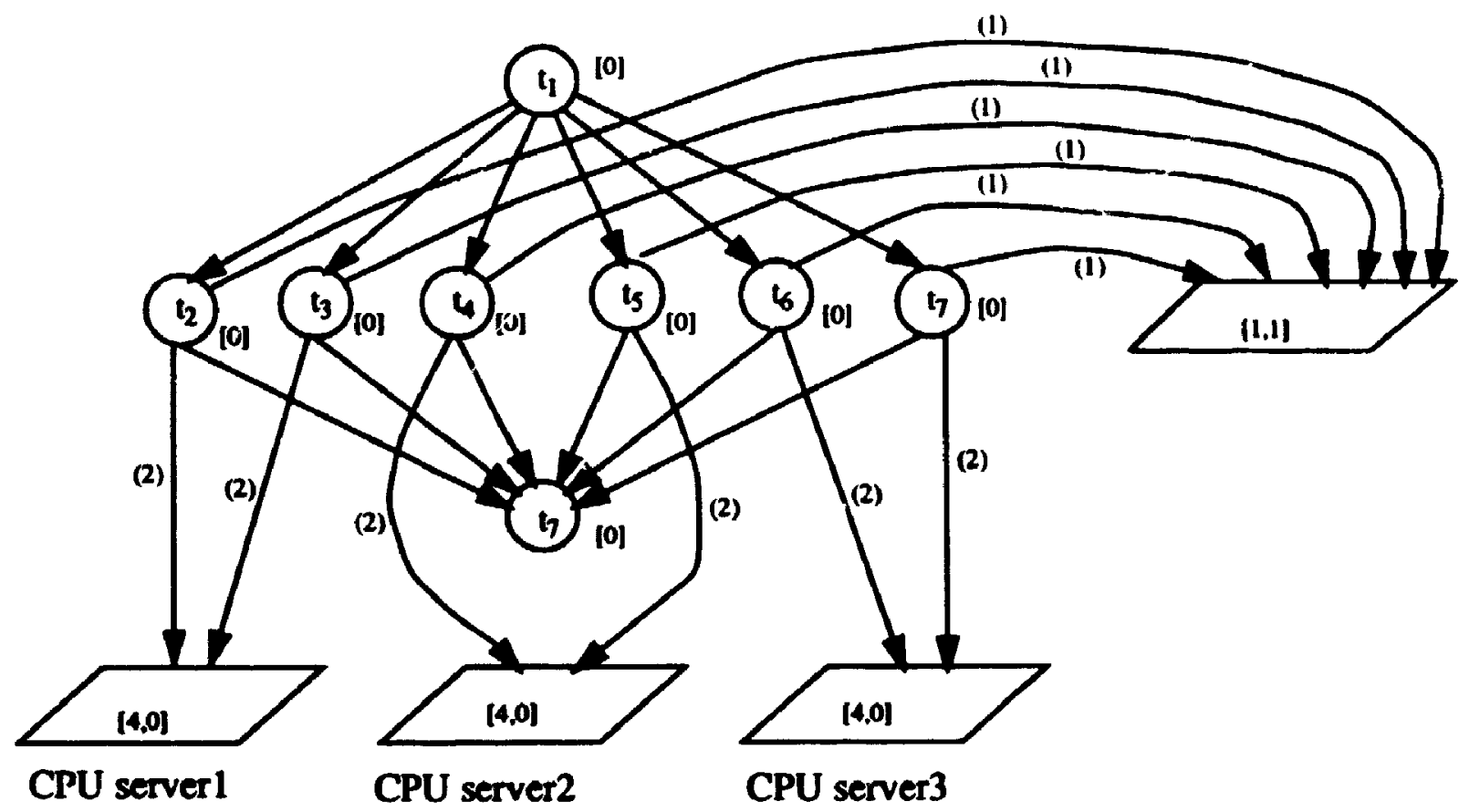

Figure 6.9. RNMTC model for processor contention

\subsubsection{Discussions}

A number of cases have been tested to examine the efficiency and accuracy of analytic solution method. The test results show that the method can be used in various kinds of system structure, such as, node precedence graph with high degree concurren :e, multiple layer fork and join, etc. The impact of processor contention can be also captured with analytic solution method by modelling processor as an SRVN server. Table 5.1 shows the minimum and maximum error rate $\varepsilon$ obtained with some of models, as well as the average of the absolute value error rate for each test suite. Most of error rates between analytic solution method result and simulation result are less than 10\%. 
Table 1.1: Simulation and Approximate results for the Balanced System (Model 1) $c(l, t)=1,(t=1, \ldots, 7) ; v(I, t, I, I)=v(I, t, 2, I)=1,(t=1, \ldots, 7) ; s(i, 1,1)=\mathrm{s}(i, 1,2)=1,(i=1,2)$; mean error rate $=3.53 \%$

\begin{tabular}{|c|c|c|c|c|}
\hline Case & $\begin{array}{c}y(i, I, I, 3, I) \\
i=I, 2\end{array}$ & approx. & simulation & $\begin{array}{c}\text { error rate } \\
(\%)\end{array}$ \\
\hline 1 & 0 & 4.2833 & 4.2841 & -0.019 \\
\hline 2 & 0.001 & 16.3116 & 16.2159 & 0.59 \\
\hline 3 & 0.2 & 16.6388 & 18.1395 & -8.273 \\
\hline 4 & 0.5 & 20.4627 & 21.9848 & -6.923 \\
\hline 5 & 0.7 & 23.5954 & 24.9417 & -5.398 \\
\hline 6 & 1 & 28.8153 & 29.7841 & -3.253 \\
\hline 7 & 1.5 & 38.2658 & 38.4570 & -0.497 \\
\hline 8 & 2 & 48.1654 & 47.6266 & 1.131 \\
\hline 9 & 3 & 68.5057 & 66.4952 & 3.023 \\
\hline 10 & 4 & 89.1406 & 85.7318 & 3.976 \\
\hline 11 & 6 & 130.7089 & 125.2500 & 4.358 \\
\hline 12 & 20 & 423.4034 & 403.5800 & 4.912 \\
\hline
\end{tabular}

Tabl: 2.1: Simulation and Approximate results when the number of concurrent tasks $=3$ (Model 2)

$$
c(I, t)=1 ; s(I, I, 1)=s(1,1,2)=1,(i=1,2)
$$

mean error rate $=2.321 \%$

\begin{tabular}{|c|c|c|c|c|}
\hline Case & $\begin{array}{c}v(i, 1,1,1) \\
i=1, \ldots, n\end{array}$ & approx. & simulation & $\begin{array}{c}\text { error rate } \\
(\%)\end{array}$ \\
\hline 1 & 0.2 & 4.4183 & 4.4465 & -0.634 \\
\hline 2 & 0.4 & 5.3154 & 5.2605 & 1.044 \\
\hline 3 & 0.6 & 6.3438 & 6.1956 & 2.393 \\
\hline 4 & 0.8 & 7.4317 & 7.2023 & 3.186 \\
\hline 5 & 1 & 8.5518 & 8.2607 & 3.525 \\
\hline
\end{tabular}


Table 2.1: Simulation and Approximate results when the number of concurrent tasks $=3$ (Model 2)

$$
\begin{gathered}
c(I, t)=1 ; s(I, 1,1)=s(I, 1,2)=1,(i=1,2) ; \\
\text { mean error rate }=2.321 \%
\end{gathered}
$$

\begin{tabular}{|c|c|c|c|c|}
\hline Case & $\begin{array}{c}v(i, 1,1,1) \\
i=1, \ldots, n\end{array}$ & approx. & simulation & $\begin{array}{c}\text { error rate } \\
(\%)\end{array}$ \\
\hline 6 & 1.2 & 9.6914 & 9.3530 & 3.618 \\
\hline 7 & 1.5 & 11.4235 & 11.0359 & 3.513 \\
\hline 8 & 3 & 20.2548 & 19.7260 & 2.681 \\
\hline 9 & 5 & 32.1679 & 31.5596 & 1.928 \\
\hline 10 & 20 & 122.0496 & 121.2140 & 0.689 \\
\hline
\end{tabular}

Table 2.2: Simulation and Approximate results when the number of concurtent tasks $=5$ (Model 2)

$$
\begin{gathered}
c(1, t)=1 ; s(1,1, I)=s(1, I, 2)=1,(i=1,2) ; \\
\text { mean ertor rate }=2.356 \%
\end{gathered}
$$

\begin{tabular}{|c|c|c|c|c|}
\hline Case & $\begin{array}{c}v(i, 1,1,1) \\
i=1, \ldots, n\end{array}$ & approx. & simulation & $\begin{array}{c}\text { error rate } \\
(\%)\end{array}$ \\
\hline 1 & 0.2 & 5.2839 & 5.3003 & -0.309 \\
\hline 2 & 0.4 & 6.9320 & 6.7660 & 2.454 \\
\hline 3 & 0.6 & 8.7498 & 8.4499 & 3.550 \\
\hline 4 & 0.8 & 10.6343 & 10.2470 & 3.780 \\
\hline 5 & 1 & 12.5529 & 12.1125 & 3.636 \\
\hline 6 & 1.2 & 14.4918 & 14.0258 & 3.323 \\
\hline 7 & 1.5 & 17.4235 & 16.9116 & 3.027 \\
\hline 8 & 3 & 32.2546 & 31.6480 & 1.917 \\
\hline 9 & 5 & 52.1679 & 51.5298 & 1.238 \\
\hline 10 & 20 & 202.0496 & 201.3940 & 0.326 \\
\hline
\end{tabular}


Table 2.3: Simulation and Approximan: results when the nurnber of concurrent tasks $=10$ (Model 2)

$$
c(l, t)=1 ; s(I, 1, l)=s(l, 1,2)=1,(i=1,2) \text {; }
$$

mean error rate $=2.546 \%$

\begin{tabular}{|c|c|c|c|c|}
\hline Case & $\begin{array}{c}v(i, I, I, I) \\
i=I, \ldots, n\end{array}$ & approx. & simulation & $\begin{array}{c}\text { error rate } \\
(\%)\end{array}$ \\
\hline 1 & 0.2 & 7.3025 & 7.0994 & 2.861 \\
\hline 2 & 0.4 & 10.9335 & 10.4546 & 4.581 \\
\hline 3 & 0.6 & 14.7489 & 14.1976 & 3.883 \\
\hline 4 & 0.8 & 18.6329 & 18.0590 & 3.178 \\
\hline 5 & 1 & 22.5515 & 21.9621 & 2.684 \\
\hline 6 & 1.2 & 26.4906 & 25.8867 & 2.333 \\
\hline 7 & 1.5 & 32.4226 & 31.8301 & 1.862 \\
\hline 8 & 3 & 62.2540 & 61.7002 & 0.898 \\
\hline 9 & 5 & 102.1676 & 101.5210 & 0.637 \\
\hline 10 & 20 & 417.6715 & 402.239 & 3.836 \\
\hline
\end{tabular}

Table 3.1: Simulation and Approximate results for the impact of client task layers (Model 3)

$$
\begin{gathered}
c(1, t)=1 ; v(1, t, I, I)=v(I, t, 2, I)=1 ; s(i, 1,1)=s(i, I, 2)=1,(i=1,3) ; \\
y(i, I, I, 3, I)=1 ; y(i, 1,2,3,1)=0
\end{gathered}
$$

mean error rate $=-3.58 \%$

\begin{tabular}{|c|c|c|c|c|}
\hline Case & $\begin{array}{c}\text { number of } \\
\text { layers }\end{array}$ & approx. & simulation & $\begin{array}{c}\text { error rate } \\
(\%)\end{array}$ \\
\hline 1 & 1 & 28.8153 & 29.7841 & -3.253 \\
\hline 2 & 2 & 56.6307 & 58.6460 & -3.433 \\
\hline 3 & 3 & 84.4460 & 87.6300 & -3.633 \\
\hline 4 & 5 & 140.0768 & 145.4340 & -3.684 \\
\hline 5 & 9 & 251.3382 & 260.9020 & -3.666 \\
\hline 6 & 12 & 334.7842 & 348.2460 & -3.866 \\
\hline
\end{tabular}


Table 4.1: Simulation and Approximate results for the impact of processor contention (Model 7 $c(l, t)=1(t=1, \ldots .8) ; v(1, t, 1,1)=v(1, t, 2, I)=1 ; s(1, I, I)=\$(I, 1,2 ;=1$; mean error rate $=2.393 \%$

\begin{tabular}{|c|c|c|c|c|}
\hline Case & $\begin{array}{c}\text { number of } \\
\text { processors }\end{array}$ & approx. & simulation & $\begin{array}{c}\text { error rate } \varepsilon \\
(\%)\end{array}$ \\
\hline 1 & 1 & 51.2278 & 51.16 & 0.132 \\
\hline 2 & 2 & 35.3333 & 38.2458 & -7.615 \\
\hline 3 & 3 & 30.3030 & 33.1757 & -8.659 \\
\hline 4 & 6 & 27.2549 & 30.1163 & -9.501 \\
\hline
\end{tabular}

Table 5.1: Accuracy of Analytic Solution

\begin{tabular}{|c|c|c|c|}
\hline Model & $\begin{array}{c}\text { Minimum } \varepsilon \\
(\%)\end{array}$ & $\begin{array}{c}\text { Maximum } \\
\varepsilon(\%)\end{array}$ & $\begin{array}{c}\text { Average } \varepsilon \\
(\%)\end{array}$ \\
\hline 1 & -0.019 & -8.273 & 3.53 \\
\hline 2 & -0.309 & 4.581 & \\
\hline 3 & -3.253 & -3.866 & $3.59 \%$ \\
\hline 7 & 0.132 & -7.615 & \\
\hline
\end{tabular}




\subsection{KNMTC Model of a Distributed Database System}

In this section, a large case study is presented, where RNMTC model is built for a distributed database Tandem On-Line Transaction Processing (OLTP) system. The starting point in building the analytic model was the simulation model described in [HMT92] which captures the hardware architecture of a Tandem computer system and software behavior of the relational database system NonStopSQL running on a Taridem multiprocessor machine [Zel90] and [Bor88]. This is called "cetailed simulation model". A set of RNMTC models are built to evaluate the performance of Tandem system by applying analytic solution. The RNMTC simulator is used to build a "abstract" model to analyze RNMTC models by simulation. The difference between the detailed and abstract simulation results gives the "abstraction error", while the difference between the abstract simulation and analytic results is called the "analytic approximation error".

\subsubsection{Tandem Distributed Database System}

A Tandem machine is a share nothing multiprocessor system on which NonStopSQL is implemented. NonStopSQL is a distributed relational database management system which achieves high performance through integration of SQL functions with operating system functions as disk VO, virtual memory, disk cache management, etc. System integration and movement of SQL functions downward from the client reduces message traffic and device utilization by putting SQL optimizations at the lower level of the system [Bor88].

Figure 6.10 shows the basic architecture of the Tandem system. Up to 16 loosely-coupled processors can be interconnected by dual high-speed buses named Dynabus to form a single multiprocessor system. The transfer rates between processors is high (over $10 \mathrm{Mb} / \mathrm{sec}$ for 8 or more processors). Based on the principles of a share nothing architecture, each CPU has its own memory and $\mathrm{VO}$ bus dedicated to interfacing to the outside world via $\mathrm{VO}$ controllers (disk controllers, terminal controllers, etc.). The data transfers on the 10 bus occurs at high speed (4 Mb/sec) through direct memory access (DMA). System resources are managed by a message-based operating system which provides communication between processes executing on the same or on different processor, making the distribution of hardware component transparent. 1/O devices are managed by system-level processes. Tandem files which contain SQL tables and indices can be horizontally partitioned across several disks into file fragments. 
The software architecture is based on a clien/server model, where client (or requesting) processes send their requests to server processes through messages, and then wait for a reply from the server. Therefore almost all communications with few exceptions is performed in a reidezvous manner. The main software components of the Tandem OLTP system modelled in [HMT92] are displayed in Figure 6.10.

Disk Process (DP) is the LO process that manages a disk volume, acting as VOO server for Giles resident on that volume. DP integrates several important operating system and database management functions: performs disk L/O through "driver" subroutines, supports access to files records, manages disk cache through cooperation with the operating system virtual memory management mechanism and implements simple SQL operations taking care of locking and of logging procedures.

Line Handler (LH) is responsible for VOO to and from the users's terminal hy providing the transmission protocol. During its operation, LH handles incoming requests and outgoing responses for up to three users.

Terminal Control Process (TCP) provides screen formatting, data validation, screen sequencing and data mapping for up to 32 terminal. TCP receives user requests from LH and forwards them to application processes. It also gets responses from the application processes and sends them back to LH. It should be mentioned that TCP uses a nonblocking communication style, being a notable exception from the RV communication style.

Pathmon provides address information to TCP for inter-process communication.

Application Process (APP) receives user requests from TCP, decomposes them into simple SQL operations which are then sent one by one to the appropriate DP for execution. After receiving the reply (or replies) from DP, APP assemblies the final response for the user and sends it back to TCP.

Executor Server Process (ESP) is an optional component used only for very complex query with potential for internal parallelism [Ze190]. In the case of partitioned files (SQL tables or indices) located on different disk volumes, several DPs can work in parallel on different processors on behalf of the same query. Thus an APP managing such a complex query will start several ESFs which will execute in parallel parts of the same query with the help of DPs. At the end, APP col- 


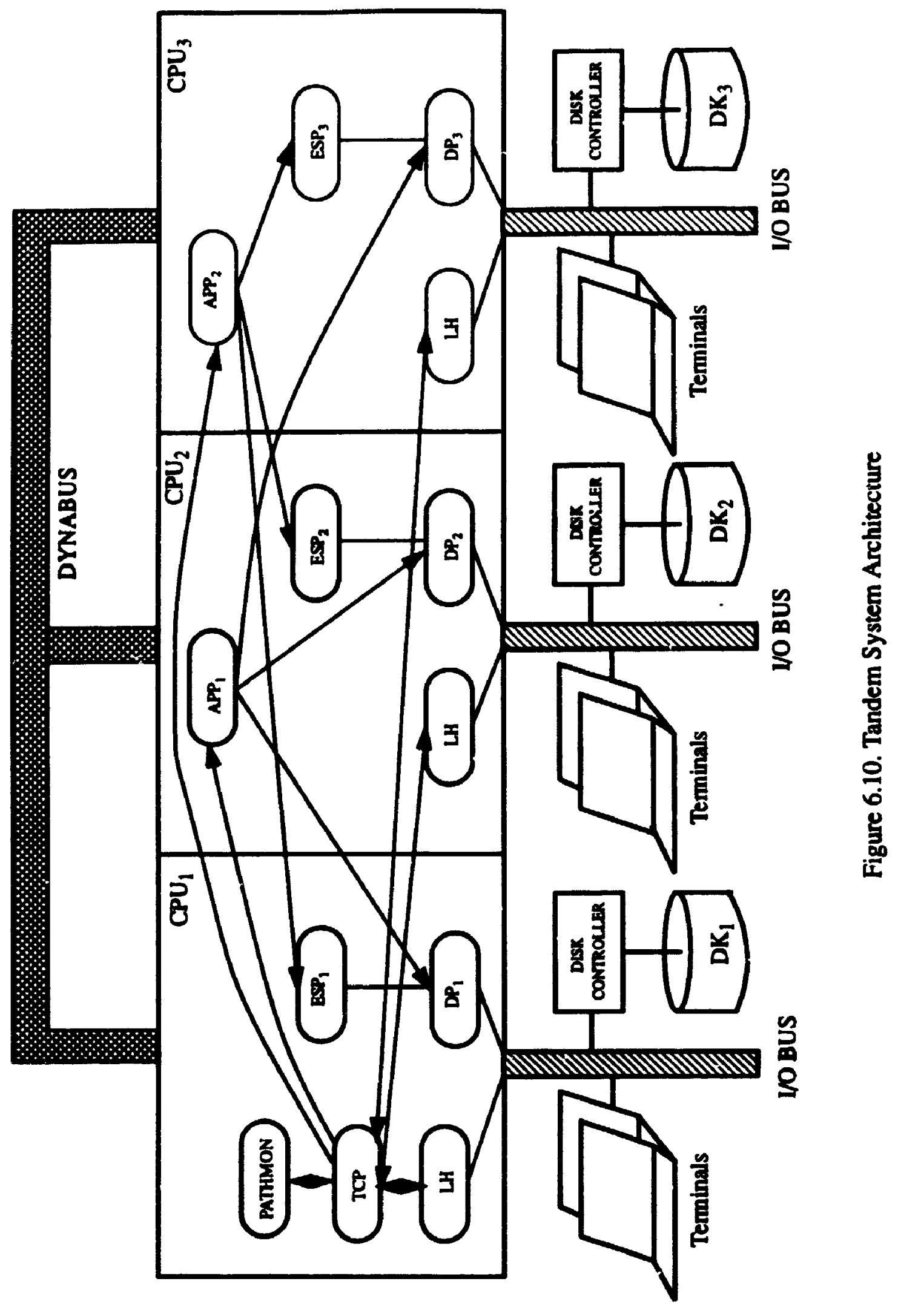


lects all partial results and assemblies the final response. For efficiency reasons, the number of ESPs used is the number of file fragments, and an ESP corresponding to a fragment runs on the same processor as the DP used to perform the low level SQL operations.

It should be mentioned that fault-tolerance, one of the important features of the Tindem architecture which is achieved by hardware and software redundancy, is not considered in the thesis.

\subsubsection{RNMTC model for Tandem System}

The RNMTC model of a Tandem database is built for two classes of client queries: light readers and join query. Some of the components of the Tandem architecture from Figure 6.10 are represented as servers and others as client tasks, depending on their roles in the system.

The hardware resources (CPU, עO devices) are naturally represented as pure servers. The software component DPs are also modelled as servers which receive service requests from APPs. In order to perform their functions DPs make in turn requests to CPUs and disks (DKs). Each DP may have several entries with different workload corresponding to different functions performed by DP (read a database block or read the primary index). The number of visits that DP makes to DK depends on the cache_hit_ratio which is a model parameter with different values for every file fragment. Each CPU also has a number of entries with different computation times corresponding to various requests. All servers in the models have only one phase which is "in RV" phase.

The remaining software processes are included in the client submodel. PATHMON, TCP. LH and APPs are modelled as client tasks which make requests to servers. In order to reduce the model size, PATHMON, TCP and LH are integrated into one node in client submodel.

The precedence graphs describing the client tasks, and the model parameters (computation time and service time, visit ratios) are determined in a way similar to the Software Performance Engineering methodology proposed by C. Smith in [Smi90]: first build software execution models for a few representative workload scenarios, then characterize their software and hardware resource requirements and then compute the parameters for the performance model of the whole system. 
In the distributed database model, the desirable performance measure is the user response time which is the time between that the user issues a query and that the user receives the answer from the system.

\subsubsection{RNMTC Model for Light Reader Operation}

The software execution model for light reader operation shown in Figure 6.11 represents a light reader that retrieves an itern from a database table based on a key value entered by the user. The database table is partitioned in three fragments located on throe different disks and the search kiy is the priniary key of the file. The query chooses one fragment depending on the primary key value from which it will retrieve the required item. The data is responded to the user.

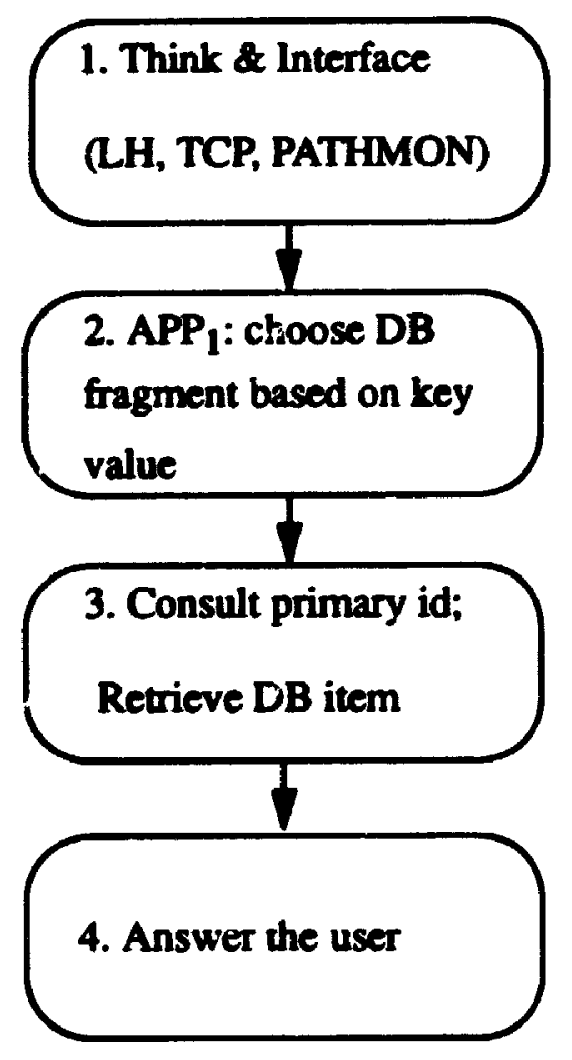

Figure 6.11. Light Reader Query Execution Model

Figure 6.12 is the RNMTC model for light reader execution model with that cache ratio is 
0.1 .

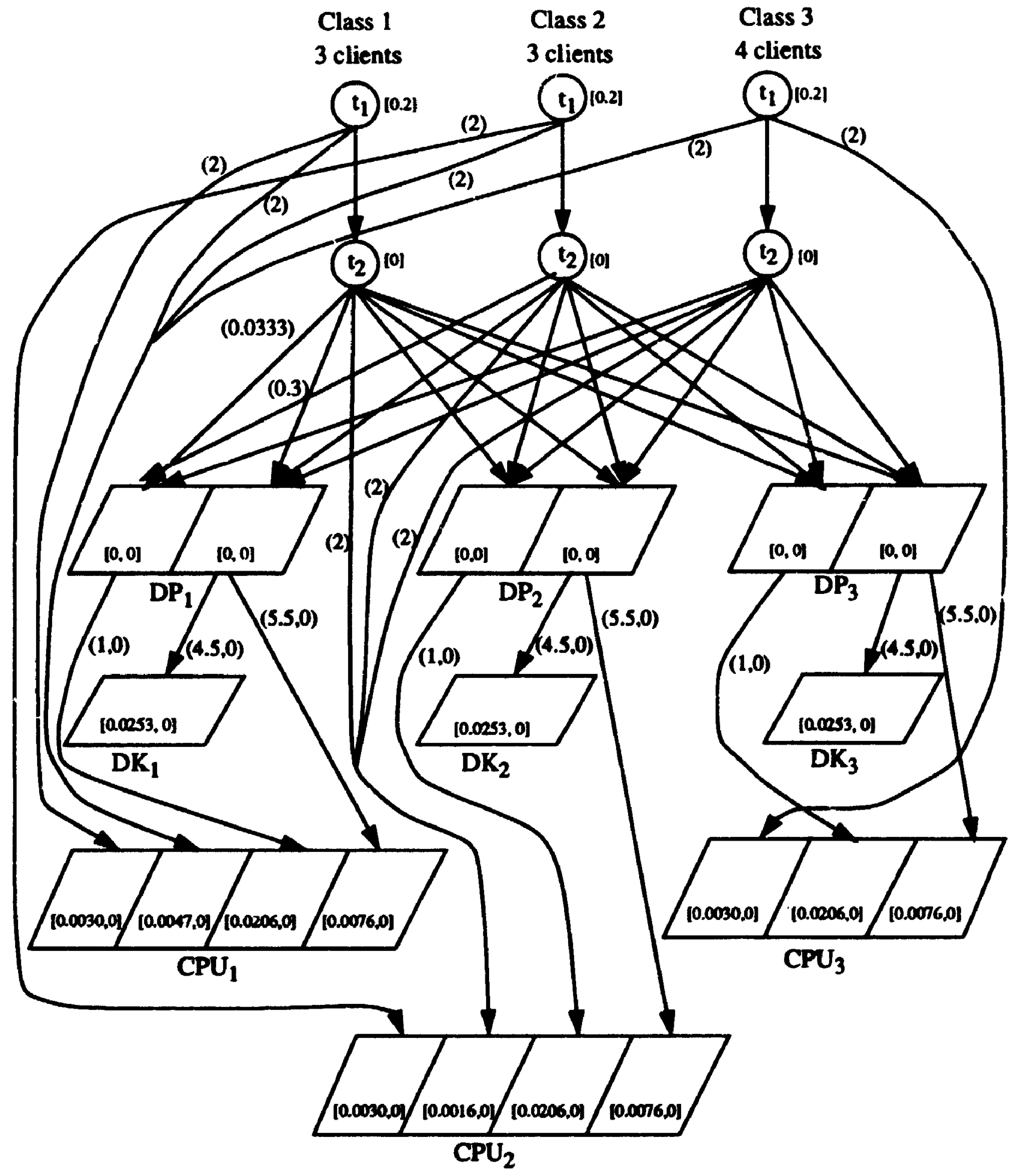

Figure 6.12. RNMTC model for light readers 
The test case includes total 10 clients. Each client submodel has two nodes executed in sequence and is single-threaded. The first node represents LH, "CP and PATHMON \%hereas the second node corresponds the other operation in the execution inodel.

The 10 clients are distributed on 3 cpus: three on $\mathrm{CPU}_{1}$, three on $\mathrm{CPU}_{2}$ and four on $\mathrm{CPU}_{3}$. Three disk processes $\left(\mathrm{DP}_{1}, \mathrm{DP}_{2}\right.$ and $\left.\mathrm{DP}_{3}\right)$ are also allosated on the three cpus. Each DP has two entries: entry 1 for the case of finding the data in cache and entry 2 for the operation of reading from DK. Each CPU also has multiple entries with different computation time for various requests either from client tasks or from servers. Figure 6.13 shows the mean response time for light readers with varied cache hit ratio.The response time is larger for a low cache hit ratio than that for a high cache hit ratio, since the chance to find a required data block in buffer is lower when cache hit ratio is low and thus move visits to the disk are necessary. The abstraction error is between $0.15 \%$ and $10 \%$, and the analytic approximation error is less than $3 \%$.

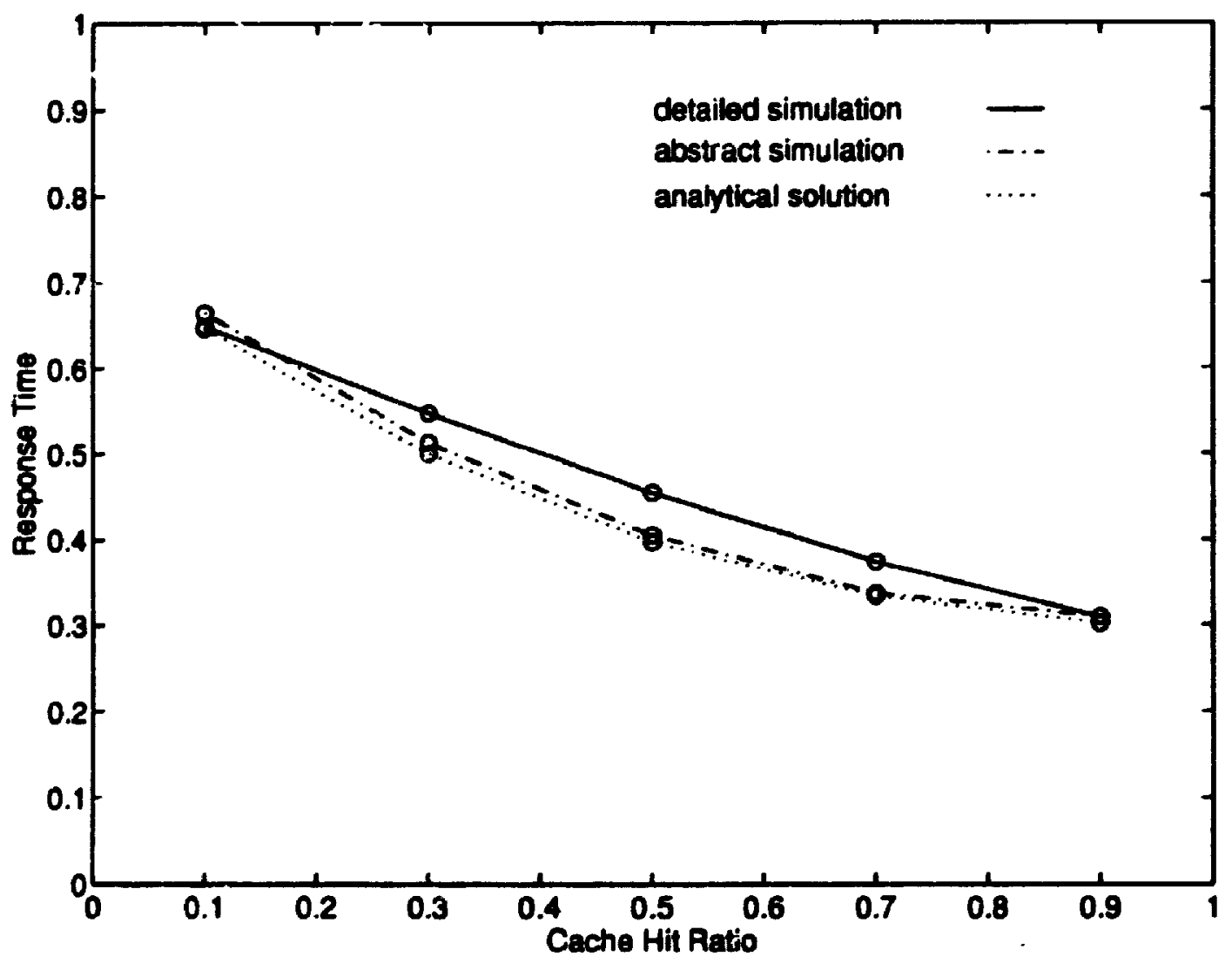

Figure 6.13. Response time in function of the cache hit ratio 


\subsubsection{RNMTC Model for Join Query Operation}

The join query operation is a heavy operation. One of the execution plans for parallel joins presented in [Zel90], assuming that a table small enough to be cached in memory is joined with a large table partitioned in three fragments residing on three different disks. Three ESP processes spawned by the application process can work in parallel. Figure 6.14 represents a join querymodel.

Figure 6.15 is the RNMTC model for parallel join query execution model. The cache hit ratio is 0.2 . There are 5 client tasks in the join client submodel. Client tasks $t_{3}, t_{4}$ and $t_{5}$ in client submodel represent the three ESPs. DPs and CPUs also have more entries than that in pure light reader model to serve different requirements both form light readers and join query user. For instance, the last entry in CPUI (workload is $[0.0140,0]$ ) is called by one of ESPs, $t_{3}$.

In this test case, we combine two classes clients in one system: one is join query, and another one is light readers. The join query user shares the system with a variable number of light readers (from 3 to 8). The model which includes two classes is shown in Figure 6.16. The cache hit ratio for join is 0.2 whereas it is 0.7 for light readers.

Figure 6.17 shows the response time for the two classes of clients. The abstraction error is less than $10 \%$ for light readers and less than $7 \%$ for join query, and the analytic approximation error is less than $3 \%$ for both classes. 


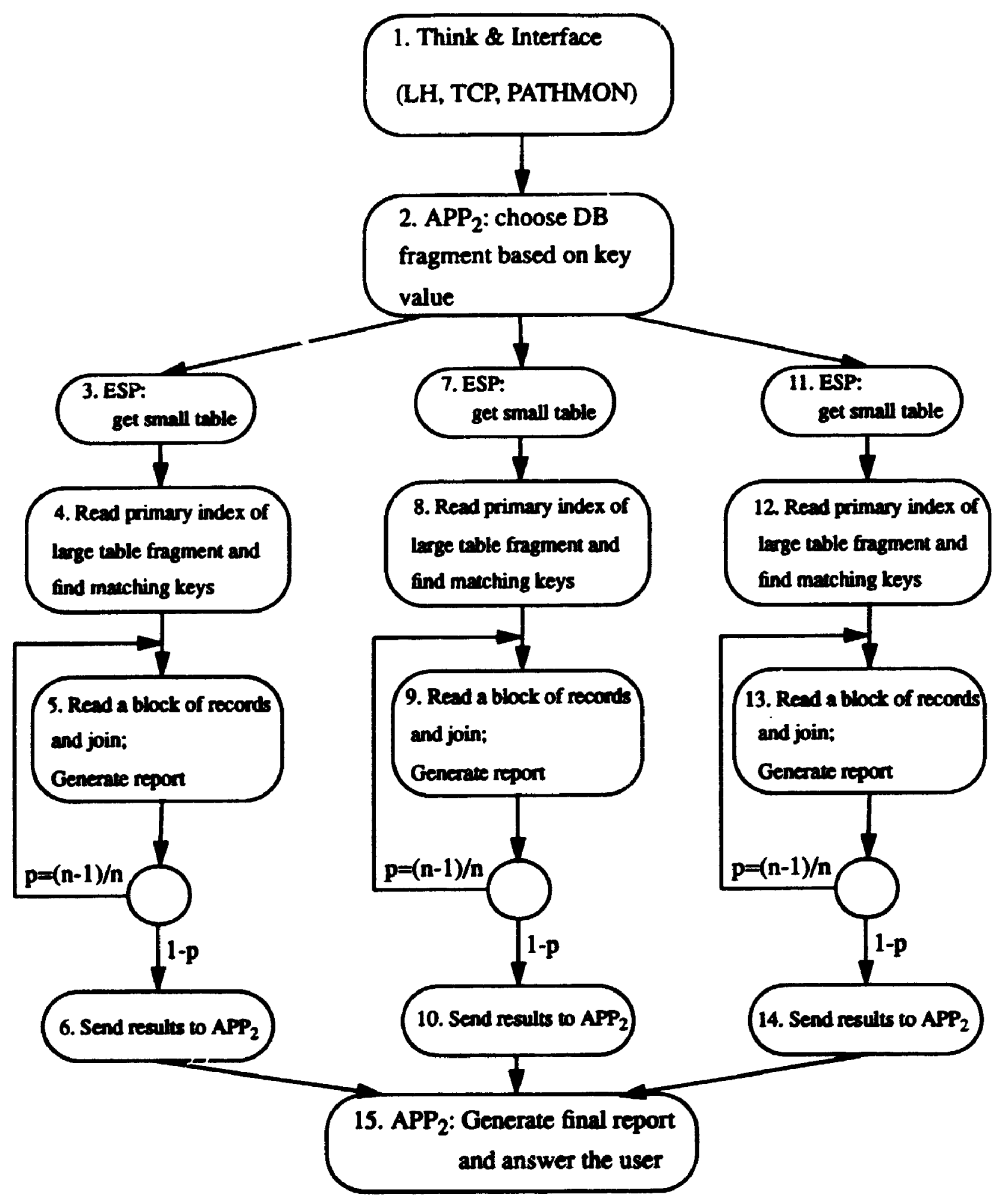

Figure 6.14. Parallel join query execution model 


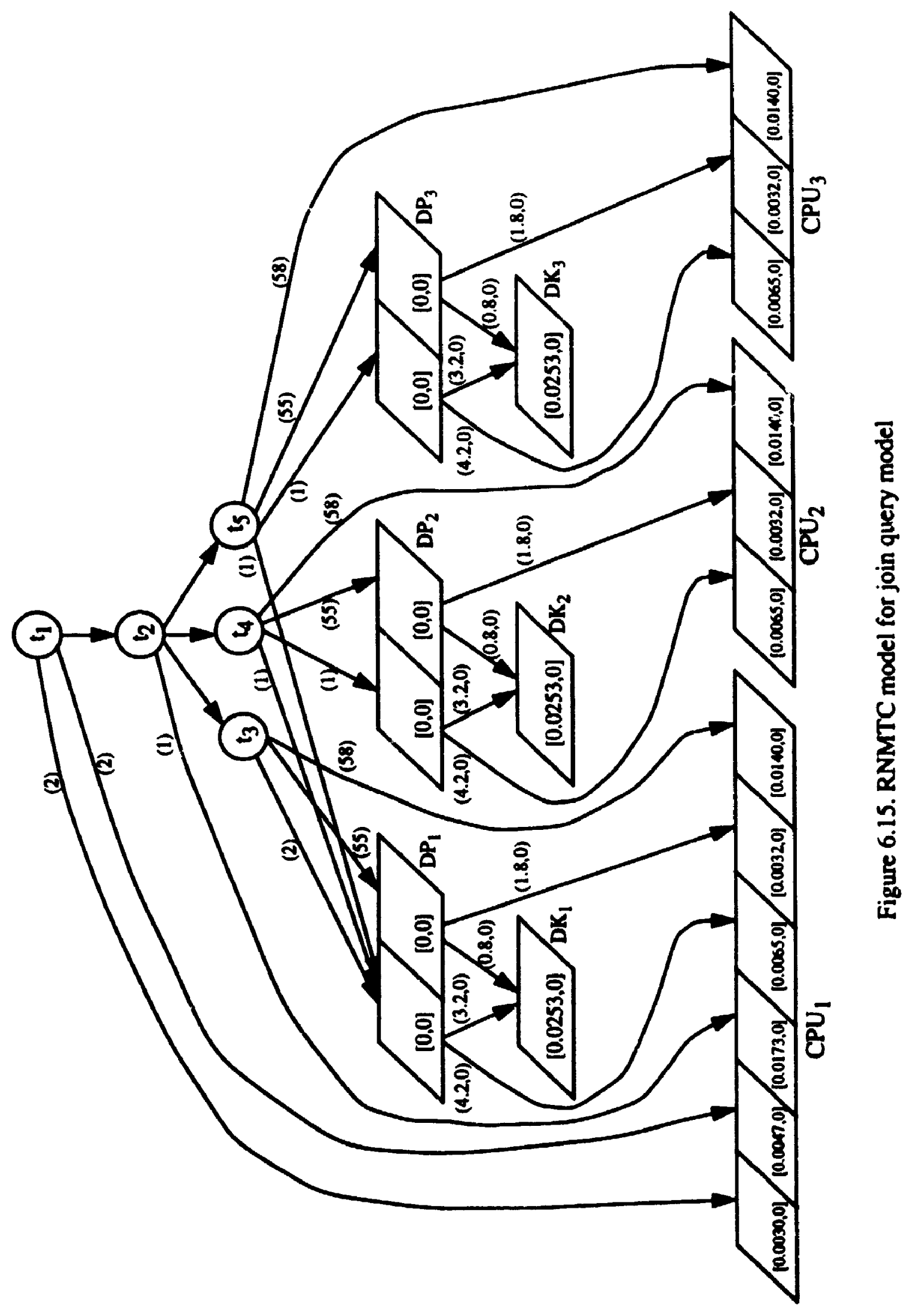




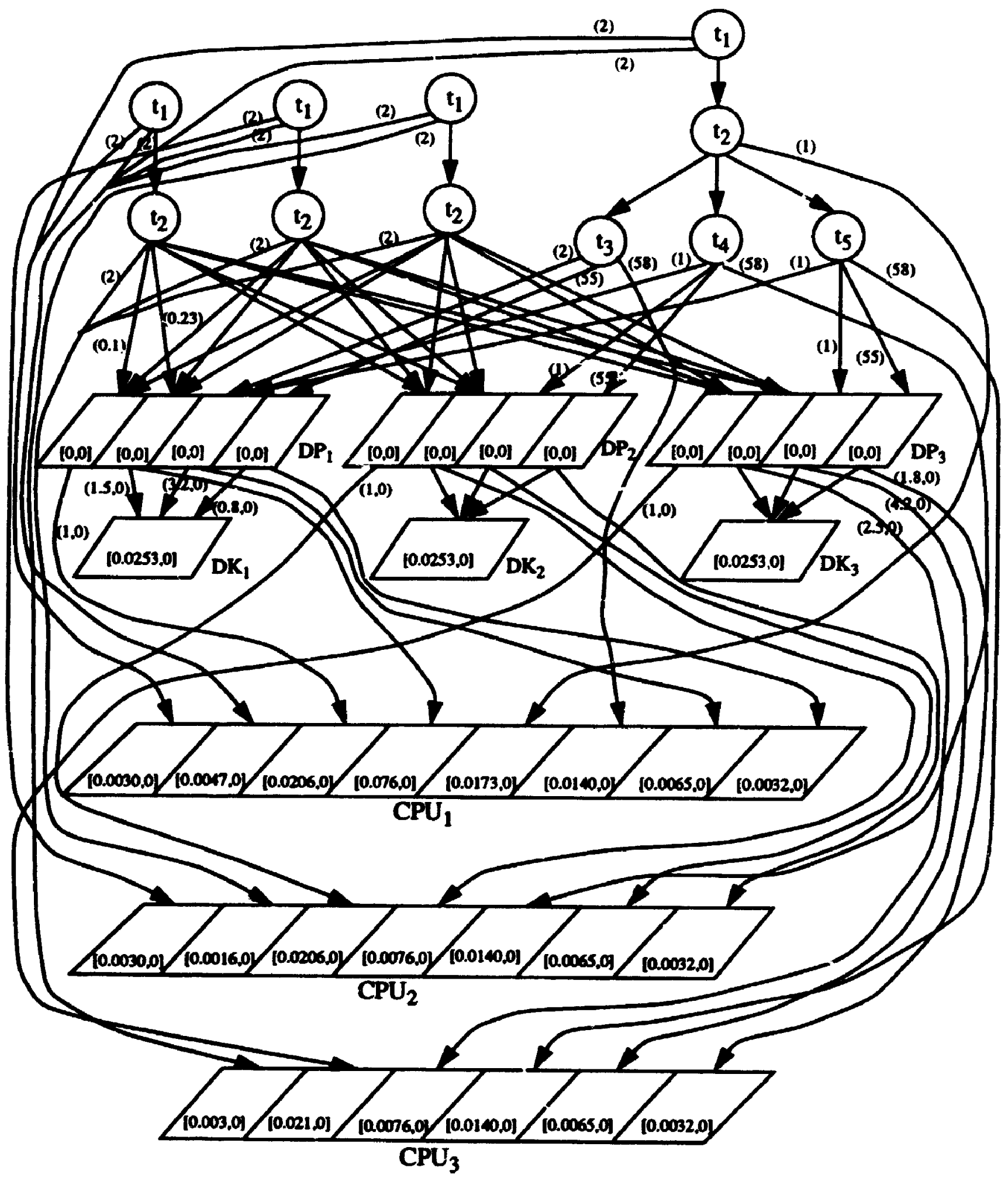

Figure 6.16. RNMTC model for two classes 


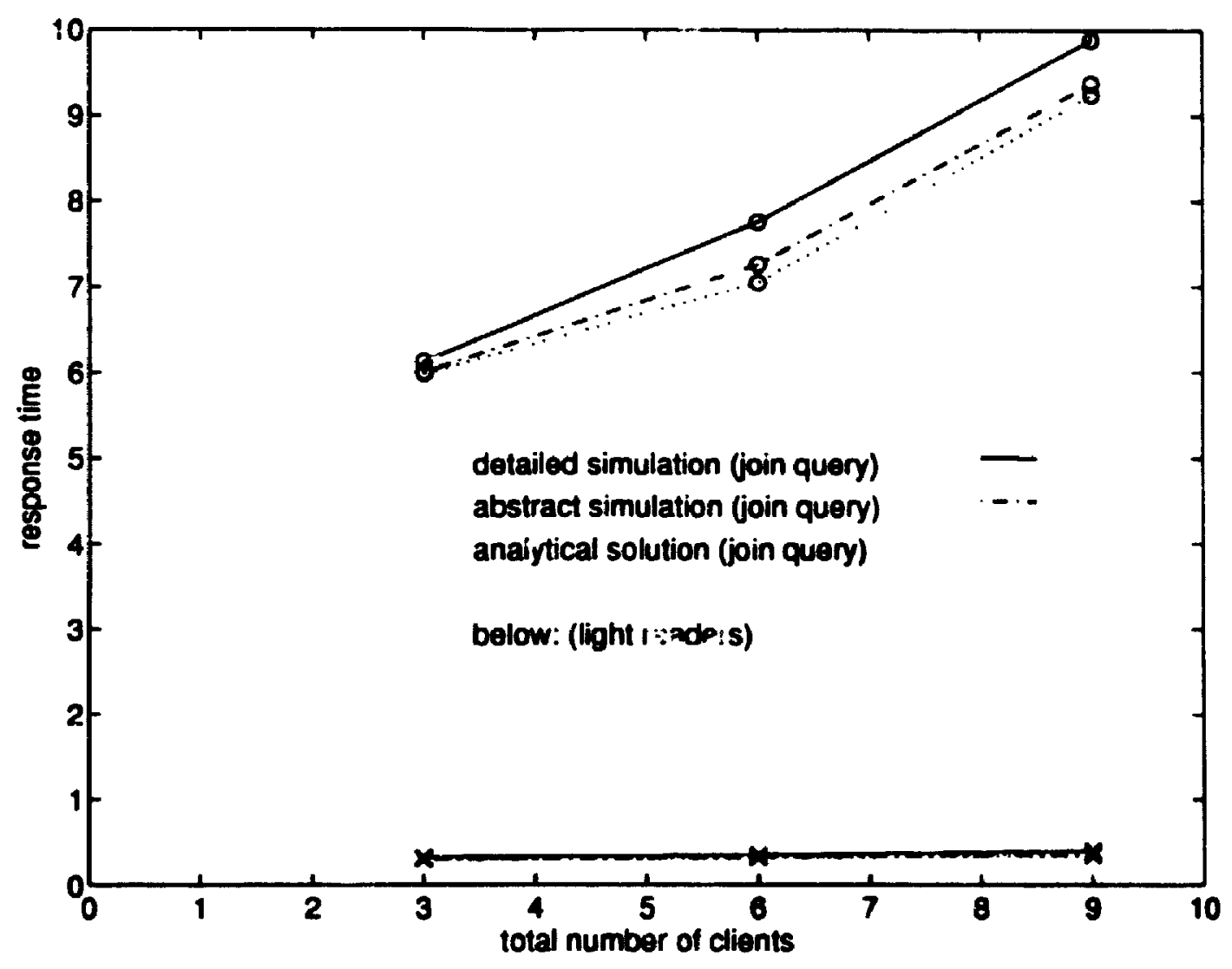

Figure 6.17. Response time for two classes of clients

\subsubsection{Hardware and Software Bottleneck}

The RNMTC model can be used to better understand the behavior of the system and to identify trouble spot that may cause performance problems. In the following example, the system behavior is studied under an incr asing number of clients (all are light readers) until approaching 
saturation. Figure 6.18 shows the increase in response time with the number of clients in the case of a cache hit ratio of 0.7 . (The approximation error is less than $3 \%$ ).

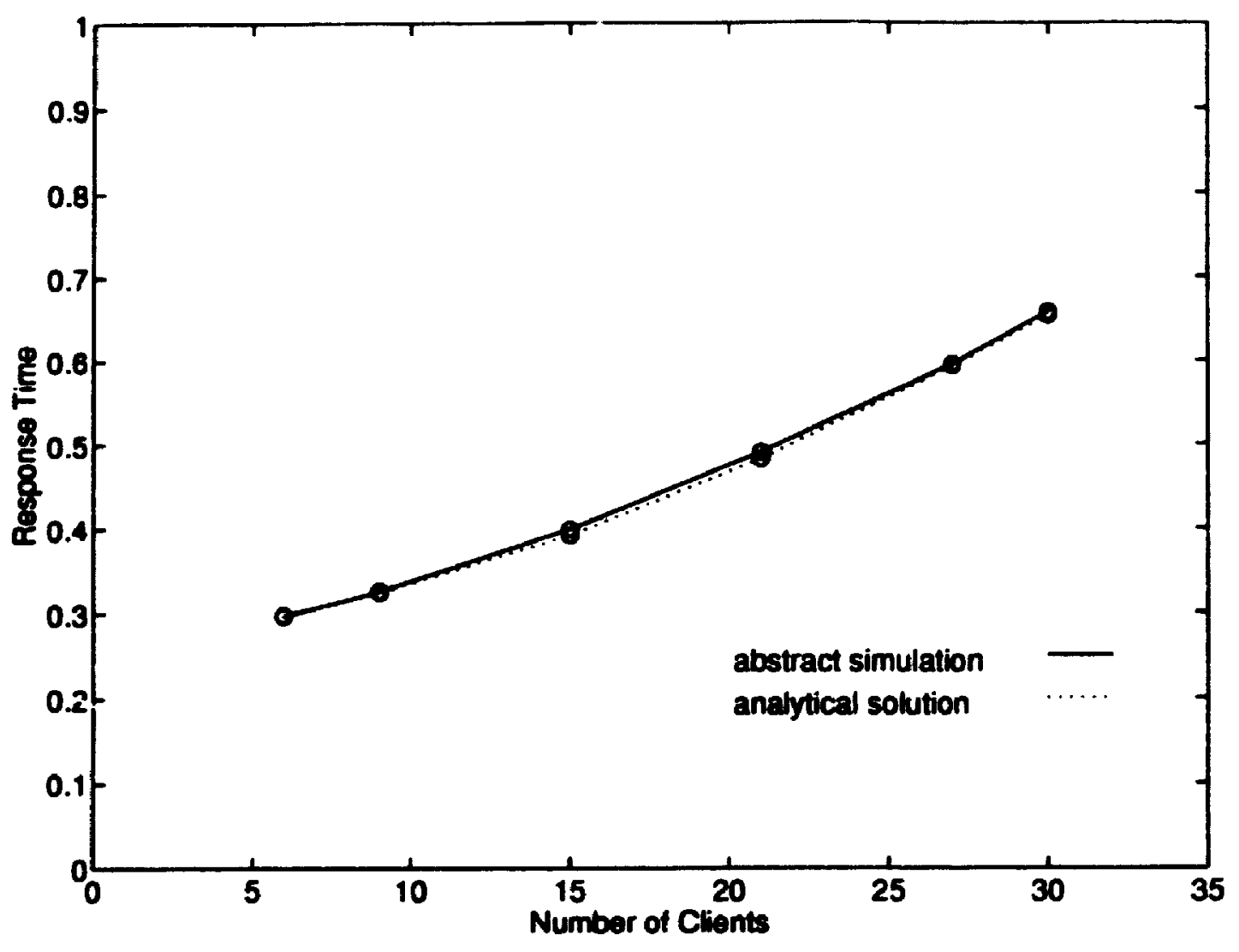

Figure 6.18. Light reader's response time in function of the number of clients

A relatively large cache hit ratio means that the number of visits to disk is relatively low, and thus the CPU is highly utilized and will become a bottleneck under heavy load. This reasoning is confirmed by Figure 6.19, where is plotted the utilization of servers $C P U_{1}, D P_{1}$ and $D K_{1}$ in function of the number of clients. 
Following a similar reasoning we could assume that, if the cache hit ratio is low than the disk will become heavily loaded and will be a potential bottleneck. However, the RNMTC results invalidate this assumption. Figure 6.20 shows the increase in server utilization with the number of clients when the cache hit ratio is 0.3 .

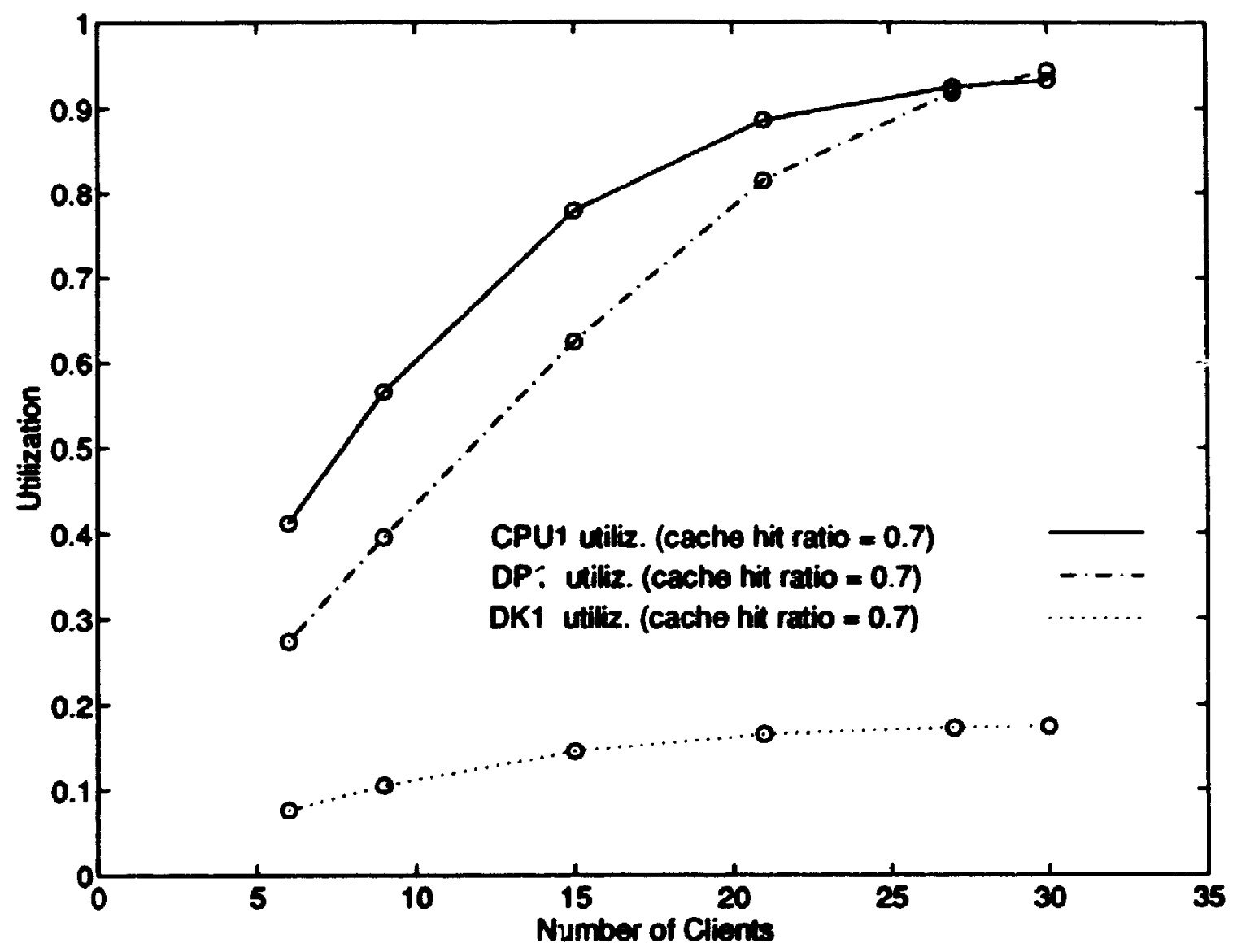

Figure 6.19. Utilization of servers in case of hardware bottleneck

None of the hardware servers is heavily loaded: both $\mathrm{CPU}_{1}$ and $\mathrm{DK}_{1}$ are utilized under $60 \%$. In this case the system bottleneck is a software server, i.e., the disk process DPI. A software bottleneck can appear in any systems where a software process plays simultaneously the role of client and server: the "busy" time as a server ineludes periods when the process itself becomes a 
client to other servers. Thus, even if the software server is blocked waiting to be served by other resources, it is nevertheless unable to accept another request from its input queue, being perceived as "busy" by its own clients. Understanding the behavior of the system helps to decide what measures are necessary to alleviate the bottleneck. The software bottleneck is a complex phenomenon involving more than one componeni, and therefore, more difficult to understand and identify than a hardware bottleneck. While the latter can be identified directly from the model parameters, the former can be diagnosed only from the results of an appropriate performance model.

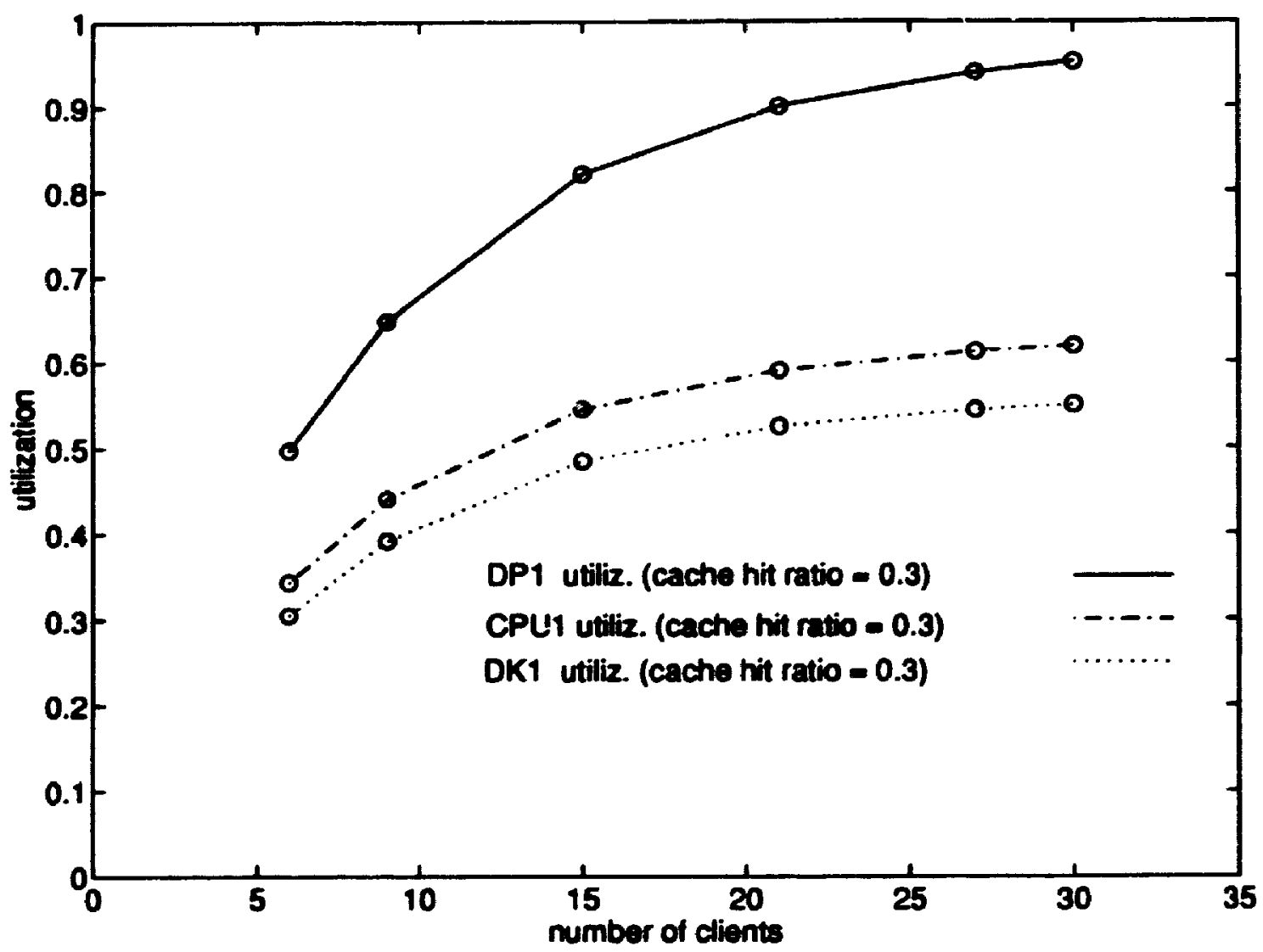

Figure 6.20. Utilization of servers in case of software bottleneck

\subsubsection{Result Analysis}

The test result for Tandem system indicates that the analytic solution is applicable for real 
system. RNMTC is used to model the distributed database system. Two simulation models were used to validate the analytic results: a "detailed simulation model" that captures the hardware architecture of a Tandem computer system and the software behavior of the relational database system NonStopSQL, and an "abstract" RNMTC simulation model. The difference between the abstract simulation and analytic results gives the "analytic approximation error". The abstraction error was found to be less than 10\%, and the analytic approximation error less than $3 \%$ for the test cases analyzed in the research. The RNMTC model of the distributed database is also used to study a case of hardware and of software bottleneck. 


\section{Chapter 7}

\section{Conclusions}

\subsection{Research Summary and Contributions}

With the increasing of system size and structure complexity, performance becomes a more and more important issue during the lifetime of system design. This research is motivated by the need for getting better analytical performance models for concurrent software systems and studying the analytic solution which has relatively high accuracy and low cost to predict and analyze system performance.

Based on a previously developed SRVN model and approximate solution for the SRVN model, this thesis developed a new model RNMTC and implemented an approximate analytic solution for this model. RNMTC is a combination of a server submodel which includes a number of servers and client submodel which is composed of a set of independent clients. The servers in the server submodel can represent both software components and hardware components and are used by the clients who compete for servers. Each system client is represented by a precedence graph and may contain multiple concurrent execution threads. An execution thread is an independent flow of control. The number of the threads can vary due to fork and join operations. The client task nodes in each client execute according to the corresponding precedence relationship. During the execution, the client task may make requests to servers. The communication between client task and server is synchronous by RV. After sending a request, the client task is blocked. Therefore the requesting execution flow is also blocked. After receiving a reply, the client task either makes another request or resume its execution. A server may make nested requests to other servers. 
The analytic solution for the RNMTC model is based on hierarchical docomposition. A MC model describes the system at the higher level to represent all possible combinations of client execution states. For each MC state an SRVN model is solved at the lower level by approximate MVA methods previously developed in [Pet91] to get state probabilities and throughputs. Finally. the higher level MC is solved to get performance measures for the system.

A fair number of test cases, both abstract and practical, are analyzed by analytic solution and the results are compared with that from simulations. The error rates for all test cases are less than $10 \%$.

Performance upper and lower bounds are also important for performance analysis. If the exact performance measures can not be derived in some cases, bounds may be the only measure for predicting system performance. Expressions for upper bounds and lower bounds are derived for some specific kinds of RNMTC model. The tightness of the bounds is also examined by comparing the bounds with simulation results.

The execution time experienced with the three analysis techniques, i.e. simuiation, analytic solution and bound method, were very different. As expected, the simulation was the longest, taking several hours for each test case (the longest was 9 hours). The analytic algorithm took one to two order of magnitude less time than the simulation, depending strongly on the size of the MC model. The running time for the computation of bounds was of a few minutes for each test case.

A paper [PMLH94] has been produced from the thesis, which will be presented at the International Workshop on Modeling, Analysis and Simulation of Computer and Telocommunication Systems, MASCOTS'94, to be held in Durham, North-Carolina, January 31 - February 2, 1994.

\subsection{Further Work}

That the exponential state explosion of the MC model suffered by analytic method for RNMTC models and the computation time required by the analytical algorithm limits its applicability to systems with a low number of clients. For example, in the case of the RNMTC model form Figure 5.16 of the database system with two classes of clients, by increasing with one the number of heavy readers, the number of the MC states grows from 2304 to over 20000 states. Future work is necessary to implement a new encoding for the MC states, aggregating similar 
states and reducing considerably the memory space required for the MC.

Another direction for future research is in the study of bounds. The expressions on bounds derived in this thesis are relatively loose, especially for some upper bounds, further work needs to be done to improve the tightness. This thesis considered a number of different RNMTC models. Extension of the bounding technique to other types of generic RNMTC model warrant further investigation. 


\section{References}

[AMBC84] M. Ajmone Marson, G. Balbo and G. Conte, "A class of generalized stochastic Petri Nets for the performance evaluation of multiprocessor systems", ACM Trans. on Computer Systems, 2(2):93-122, May 1984

[BNR88] Bell-Northern Research Ltd., Ottawa, Canada, BNR Prolog User Guide, 1988.

[Bor88] A. J. Borr, "High performance SQL through low-level system integration," ACM. pp. 342-349, 1988.

[DG92] D. Dewitt and J. Gray, "Parallel database systems: the future of high performance database systems," CACM, vol. 35, pp. 85-98, June 1992.

[FB73] E. B. Fernandez and B. Bussell, "Bounds on the number of processors and time for multiprocessor optimal schedules," IEEE transactions on computers, vol. C-22. NO. 8, pp. 745-751,August 1973.

[HL87] V-T. Hsieh and S. S. Lam, "Two classed of performance bounds for closed queueing networks," Performance Evaluation, vol. 7, pp. 3-30, March 1987.

[HMT92] C. Hrischuk, E. Melin and A. Thioliere, "Transaction processing simulator user's guide," SCE-92-23, Department of systems and somputer engineering, Carleton University, December 1992.

[HT83] P. Heidelberger and K. S. Trivedi, "Analytic queveing models for programs with internal concurrence," IEEE Transactions on Computers, vol. C-32, pp. 73-82, January 1983.

[Kle] L. Kleinrock, "Queueing system"

[KME89]A. Kapelnikov, R. R. Muntz, and M. D. Ercegovac, "A modeling methodology for the analysis of concurrent systems and computations," Journal of Parallel and Dis. tributed Computing, no. 6, pp. 568-597, 1989.

[KME92] A. Kapelnikov, R. R. Muntz, and M. D. Ercegovac, "A methodology for perfor- 
mance analysis of parallel computations with looping constructs," Journal of Par. allel and Distributed Computing, no. 14, pp. 105-120, 1992.

[Lak87] M. S. Lakshmi, "Performance engineering of a videotex system," Proceedings of the twentieth annual hawall international conference on system sciences, pp. 363-370, 1987.

[Lav89] S. S. Lavenberg, "A perspective on queueing models of computer performance," Performance Evaluation, vol. 10, pp. 53-76, 1989.

[LZGS84] E. D. Lazowska, J. Zahorjan, G. S. Graham and K. C. Sevcik, "Quantitative System Performance", Prentice-Hall Inc., Englewood Cliffs, N.J. 07632, 1984

[MBC84] M. Ajmone Marsan, G. Balbo, and G. Conte, "A class of generalized stochastic Petri Nets for the performance evaluations of multiprocessor systems," ACM Trans. on Computer Systems, vol. 2, pp. 93-122, May 1984.

[Mol82] M. K. Molloy, "Performance analysis using stochastic Petri Nets", IE.GE Trans. on Computers, C-31:913-917, September 1982

[MWNP88] J. W. Miernik, C. M. Woodside, J. E. Neilson and D. C. Petriu, "Throughput of stochastic rendezvous networks with caller-specific service and processor contention," in Proceeding IEEE Infocom '88, pp. 1040-1049, March 1988.

[MWNP89] J. W. Miernik, C. M. Woodside, J. E. Neilson and D. C. Petriu, "Throughput of stochastic rendezvous networks with priority tasks," in Performance of Distributed and Parallel Systems (T. Hasegawa, H. Takagi and Y. Takahashi, eds.), pp. 511525, Elsevier Science Publisher, 1989.

[MWNP91] S. Majumdar, C. M. Woodside, J. E. Neilson and D. C. Petriu, "Performance bounds for concurrent software with rendezvous," Performance Evaluation, vol. 13, pp. 207-236, 1991.

[MWNP92] S. Majumdar, C. M. Woodside, I. E. Neilson and D. C. Petriu, "Robust box bounds: Network performance guarantees with minimal stochastic asstimptions," May 1992. 
[Nei_1] J. E. Neilson, "PARASUL Users' manual," School of Computer Science, Carleton University, Ottawa, Canada.

[Nei_2] J. E. Neilson, "A PARASOL tutorial," School of Computer Science, Carleton University, Ottawa, Canada.

[NW88] L. M. Le Ny and C. M. Woodside, "Performance modelling of queues with rendezvous service," Tech, Rep. No. 941, Unite de Recherche INRIA - Rennes, Centre National de la Recherche Scientifique, Universite de Rennes, France, 1988.

[Pet91] D. C. Petriu. “Approximate solution for stochastic rendezvous networks by Markov cahin task-directed aggregation," Ph. D thesis, SCE-91-21, Department of systems and somputer engineering, Carleton University, 1991

[Pet93] D. C. Petriu, "Approximate Mean Value Analysis of Client-Server Systems with Multi-class requests", SCE-93-30, Real-Time and Distributed Systems Group, Carleton University, 1993. Submitted to SIGMETRICS‘ 94.

[PMLH94] D. C. Petriu, S. Majumdar, Jingping Lin and Curtis Hrischuk, "Analytic Performance Estimation of Client-Server Systems with Mutil-Threadded", SCE-93-15, Real-Time and Distributed Systems Group, Carleton University. Accepted at MAS. COTS`94, Durham NC, January 1994

[PS87] Dartzen Peng and Kang G. Sehin, "Modeling of concurrent task execution in a distributed system for real-time control," IEEE transactions on computer, vol. C36, N). 4, April 1987.

[PW91] D. C. Petriu and C. M. Woodside, "Approximate MVA for software client/server model by Markov chain task-directed aggregation," In Proc. of The Third IEEE Symposium on Parallel and Distributed Processing, (Dallas, 'Texas), December 1991.

[PW93] D. C. Petriu and C. M. Woodside, "A New Mean Value Analysis of Client-Server Software by Task-Directed Aggregat", SCE-93-29, Real-iime and Distniouted Systems Group, Carleton University, 1993 
[Rad84] S. Radia, "A Markov model for distributed communicating sequential processes," in Performance '84, Proceedings of the 10th International Symposium on Computer Performance, pp. 3-16, North Holland, 1984.

[Rol88] J. A. Rolia, "Performance estimates for systems with software servers: The lazy boss method," Proceedings VII SCCC International Conference on Computer Science, pp. 25-43, July 1988.

[Sin92] Alok Sinha, "Client-server computing," CACM, vol. 35, pp. 77-89, July 1992.

[Smi90] Connie U. Smith, "Performance Engineering of Software Systems", 1990, AddisonWesley Publishing Company, Inc.

[TB86] A. Thomasian and P. Bay, "Analytic queueing network models for parallel processing of task systems," IEEE Transactions on Computers, vol. C-35, pp. 10451054, December 1986.

[Tho85] A. Thomasian, "Performance analysis of task systems using a queueing network model," IEEE, pp. 234-242, 1985.

[VZL87] M. K. Vernon, J. Zahorjan, and E. D. Lazowska, "A comparison of performance Petri Nets and Queueing Network models", In S. Fida and G. Pujolle, editors, Modelling Techniques and Performance Evaluation, pages 191-202. Elsevier Science Publishers B. V. (North-Holland), 1987

[WNMP88] C. M. Woodside, J. E. Neilson, J. W. Miemik and D. C. Petriu, "Performance of concurrent rendezvous systems with complex pipeline structures," in Proc. 4th International Conference in Modelling Techniques and Tool for Computer Performance Evaluation, pp. 361-378, September 1988.

[WNPM89] C. M. Woodside, J. E. Neilson, D. C. Petriu and S. Majumdar, "The rendezvous network model for performance synchronous multi-tasking distributed software," SCE89-8, Dept. of Systems and Computer Engineering and school of Computer Science, Carleton University, March 1989.

[Woo89] C. M. Woodside, "Throughput calculation for basic stochastic rendezvous net- 
works," Performance Evaluation, vol. 9, pp. 143-160, 1989

[Zel90] H. Zeller, "Parallel query execution in NonStop SQL," IEEE, pp. 484-487, 1990. 


\section{Appendix}

\section{Usage of RNMTC Simulator and analytic Solver}

\section{Directories}

All the packages are in directory: jingping/mmtc.

Under -jingping/mmtc, there are two subdirectories: "analysis" which includes the analytic solver and "simulation" which includes the RNMTC simulator.

\section{Usage of RNMTC Simulator}

Under jingping/mmtc/simulation, there are two subdirectories: "data" which includes the data of the simulation system and "source" which includes the source code of simulator.

File "Makefile" is for compiling the source code and generating executable code. There are two executable codes should be generated: "datainput" and "mtms.sun4". To create them, do the following under jingping/mmtc/simulation:

1. make datainput

2. make mtms.sun4

\subsection{Data Input}

Firsi, the data for the system under simulation must be built. In order to do this, type:

"datainput" under $\sim$ jingping/rnmtc/simulation. 
After typing this, you will be promoted for the data, such as task name, server name, execution time and precedence relationship. The task name and server name are digits and can not be same for different tasks in the same client.

"model name" is the test suite number which you will use when you run the simulator.

The data will be saved in jingping/mmtc/simulation/data.

Note: please check the limit for each data in source code " jingping/mmic/simulation/ source/datainputc", so the input data will not exceed the limit.

\subsection{Simulation}

After inputting the data, the simulation cain be started. To start simulation, type:

"mtms.sun4" under -jingping/rnmtc/simulation.

When asked "model name", input the model name which is under simulation.

The information how to decide the simulation random seeds, period and etc. can be referenced in [Nei_1] and [Neil_2].

\section{Usage of RNMTC analytic Solver}

Under jingping/mmtc/analysis, there are two subdirectories: "data" which includes the data of the system and "source" which includes the source code of the solver.

File "Makefile" is for compiling the source code and generating executable code. There are two executable codes should be generated: "datainput" and "Algo". To create them, do the following under jingping/mmtc/analysis:

1. make datainput

2. make Algo

\subsection{Data Input}

The data input for analytic solver is very similar with that in simulator. The data is stored in 
-jingping/mmtc/analysis/data.

3.2. Start Algo

After inputting the data, you can use the solver to get the performance measures for a certain model, to do so, type:

"Algo" under -jingping/mmtc/analysis.

When asked "model name", input the model name which is under tested. 

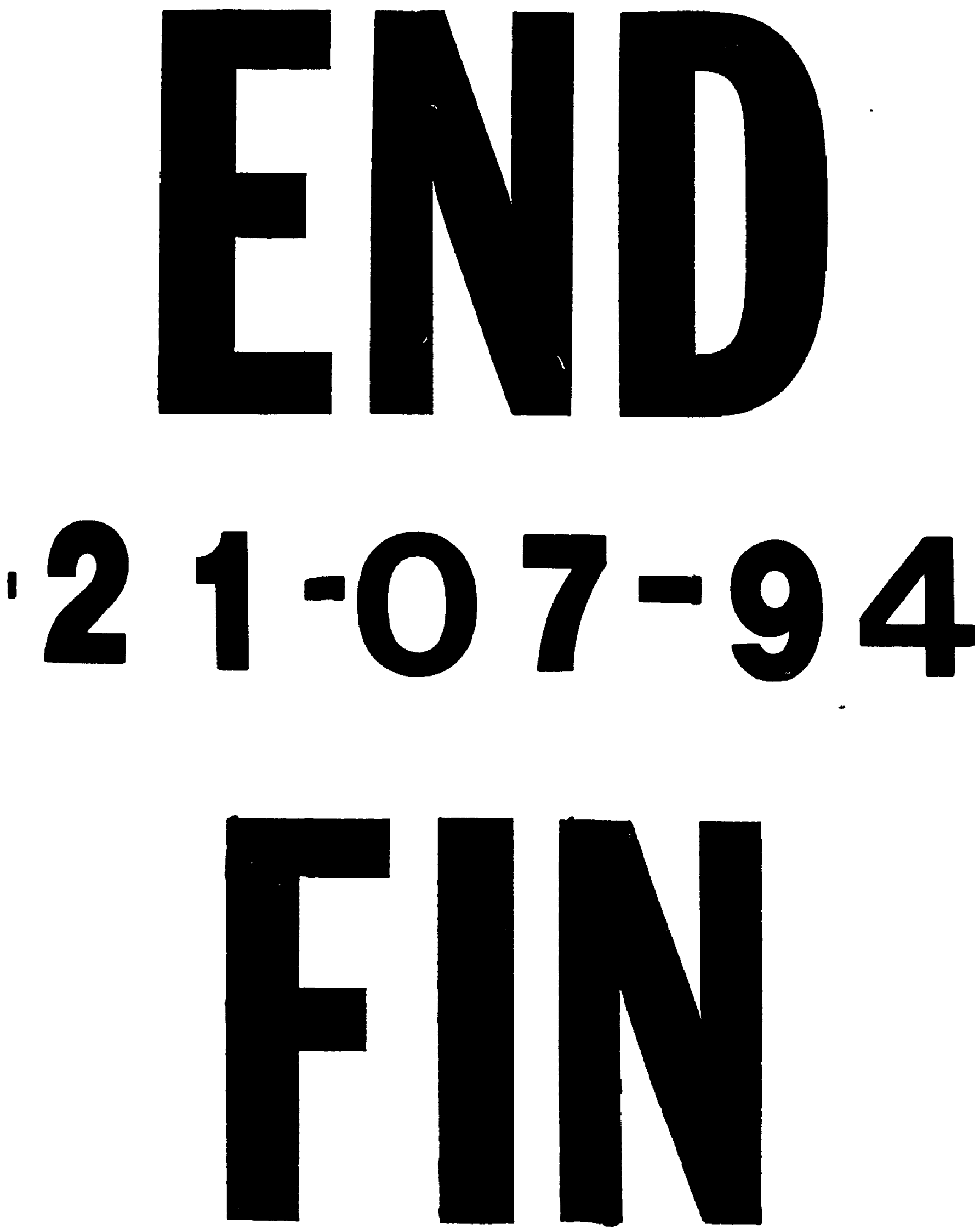